Sahinyan et al. 2021 manuscript

\title{
Application of ATAC-Seq for genome-wide analysis of the chromatin state at single myofiber resolution
}

\author{
Korin Sahinyan $^{1,2 *}$, Darren M. Blackburn ${ }^{1,2 *}$, Marie-Michelle Simon ${ }^{1,3 *}$, Felicia Lazure ${ }^{1,2}$, \\ Tony Kwan ${ }^{1,3}$, Guillaume Bourque ${ }^{1,3,4}$, Vahab D. Soleimani ${ }^{1,2,5}$ \\ ${ }^{1}$ Department of Human Genetics, McGill University, 3640 rue University, Montréal, QC, H3A OC7, \\ Canada. \\ ${ }^{2}$ Lady Davis Institute for Medical Research, Jewish General Hospital, 3755 Chemin de la Côte-Sainte- \\ Catherine, Montréal, QC, H3T 1E2, Canada. \\ ${ }^{3}$ McGill University and Genome Québec Innovation Centre, Montréal, QC, Canada. \\ ${ }^{4}$ Canadian Centre for Computational Genomics, Montréal, QC, Canada; Institute for the Advanced \\ Study of Human Biology, Kyoto University, Kyoto, Japan \\ ${ }^{5}$ Corresponding author: Tel: +1 5143408222 ext. 26136 Fax: +1 5143407502 \\ vahab.soleimani@mcgill.ca \\ " These authors have contributed equally.
}


Sahinyan et al. 2021 manuscript

ABSTRACT

Myofibers are the main components of skeletal muscle, which is the largest tissue in the body. Myofibers are highly adaptive and can be altered under different biological and disease conditions. Therefore, transcriptional and epigenetic studies on myofibers are crucial to discover how chromatin alterations occur in the skeletal muscle under different conditions. However, due to the heterogenous nature of skeletal muscle, studying myofibers in isolation proves to be a challenging task. Single cell sequencing has permitted the study of the epigenome of isolated myonuclei. While this provides sequencing with high dimensionality, the sequencing depth is lacking, which makes comparisons between different biological conditions difficult. Here we report the first implementation of single myofiber ATAC-Seq, which allows for the sequencing of an individual myofiber at a depth sufficient for peak calling and for comparative analysis of chromatin accessibility under various physiological and disease conditions. Application of this technique revealed significant differences in chromatin accessibility between resting and regenerating myofibers, as well as between myofibers from a mouse model of Duchenne Muscular Dystrophy (mdx) and wild type (WT) counterparts. This technique can lead to a wide application in the identification of chromatin regulatory elements and epigenetic mechanisms in muscle fibers during development and in muscle-wasting diseases.

\section{INTRODUCTION}

45 Skeletal muscle evolved for contraction and the production of force. The main component of skeletal muscle are myofibers which are formed from the fusion of myogenic precursor cells (1) resulting in large postmitotic syncytia that are composed of repeating contractile units, called sarcomeres (2). Myofibers exhibit wide variations in their metabolic activity and contractile properties (3). In addition, they have a highly adaptive nature where their size, myosin heavy chain isoform, energy metabolism and the overall skeletal muscle mass, among other characteristics, are regulated by complex processes involving rates of protein turnover (4,5), as well as transcriptional (6) and posttranscriptional (7) control of gene expression. Due to their adaptive nature, myofibers can change in response to exercise $(3,8,9)$, aging (10) and diseases, such as sarcopenia $(11,12)$ and cachexia (13). Therefore, the study of the myofiber transcriptome and epigenome can provide key insights into how skeletal muscle adapts and changes under various stimuli, and it can potentially lead to the discovery of novel therapeutic venues for muscle related diseases. 
Sahinyan et al. 2021 manuscript

myogenic cell types (18). Previous studies using whole muscle sequencing captures not only the myofibers but also the other resident cell types in the muscle, making it challenging to attribute any changes in the transcriptome and epigenome specifically to myofibers as they could be due to changes in these other cell types. Recent advances in Next Generation Sequencing (NGS) now allow for high dimensional analysis at a single cell level. Recent studies using these technologies to study muscle tissue, such as single nucleus RNA-Seq and ATAC-Seq have analyzed the transcriptome and epigenome of the myonuclei within the muscle fiber (19-21). However, they present certain limitations where they sequence all myonuclei present in the muscle and cannot distinguish between different myofibers as well as having low sequencing depth with a limited capacity for downstream analyses.

The chromatin state plays a key roles in transcriptional regulation and the determination of cellular identity (22). Although the accessible regions make up only $3 \%$ of the total genome, it represents over 90\% of known transcription factor binding sites (23). Chromatin accessibility is a determinant of gene expression and changes in chromatin accessibility have been identified in different biological and disease conditions such as during development $(24,25)$, cancers $(26,27)$ and neurological disorders $(28,29)$. Thus, in recent years, the study of epigenetics and chromatin accessibility has become a promising field for the development of novel therapeutics. Today, ATAC-Seq is a widely used method that allows for the mapping of the accessible chromatin regions in the genome. ATAC-Seq relies on the hyperactive Tn5 transposase that fragments the accessible regions in the genome while simultaneously ligating sequencing compatible adaptors $(30,31)$. Over the years, ATAC-Seq has been applied to many different cell types and tissues (32-34). However, to our knowledge, it has not been performed on a single myofiber, possibly due to the rigidity of their membrane, high levels of mitochondria (35-37) and the low number of myonuclei that are present in a single myofiber $(38,39)$.

Here, we have adapted OMNI-ATAC-seq to determine the genome-wide chromatin accessibility of myonuclei contained within a single Extensor Digitorum Longus (EDL) muscle fiber of a mouse. The single myofiber ATAC-Seq (smfATAC-Seq) method that we applied in this study allows for the investigation of the accessible chromatin regions of a single myofiber, without the presence of other confounding cell types. The smfATAC-Seq has a sequencing depth of approximately 6 million final reads aligned that provides approximately 30000 peaks called. Using this method, we provide comparative analysis of chromatin accessibility between resting and regenerating myofibers, as well as their MuSC progenitors. In addition, application of this method to the study of myofibers isolated from a mouse model of Duchenne Muscular Dystrophy (mdx) (40) and their WT counterparts provide a genome-wide assessment of changes in chromatin accessibility in Duchenne Muscular Dystrophy (DMD). This method can be used in the future to profile the epigenetic state of myofibers in different disease conditions, and 
Sahinyan et al. 2021 manuscript

93 under various physiological and physical stimuli, and to identify active cis-regulatory elements in muscle

94 fibers.

95 RESULTS

96 Generation of ATAC-Seq libraries from a single myofiber

97 A single EDL myofiber of a mouse contains an average of 200-300 myonuclei $(38,39)$, making genome-

98 wide analyses of the chromatin state difficult. With the advancements in next generation sequencing

99 (NGS) and the development of the OMNI ATAC-Seq protocol (30), analysis of chromatin accessibility of

100 samples with an input of as low as 500 cells is now possible (30). However, myofibers present additional

101 challenges with their rigid membrane and high levels of mitochondria (35-37). Here we report a robust

102 protocol for the successful application of ATAC-Seq on a single myofiber isolated from the EDL muscle.

103 Our method relies on the lysis and permeabilization of a single myofiber followed by transposition with a

104 hyperactive Tn5 transposase (30) (Figure 1). DNA fragment sizes obtained from the smfATAC-Seq were

105 of a similar range in size as those obtained from conventional OMNI ATAC-Seq that we have performed

106 on 5000 MuSCs that were freshly sorted by Fluorescence Activated Cell Sorting (FACS) (Figure 1-Figure

107 Supplement 1). Furthermore, analysis showed that only 0.9-2.09\% of reads were derived from the

108 mitochondria in smfATAC-Seq (Table 1), suggesting that this method is highly efficient for the removal

109 of mitochondria from mitochondria-rich myofibers. Following the removal of mitochondrial reads, there

110 were approximately 6 million final reads aligned and 30000 peaks called, demonstrating a sufficient

111 sequencing depth for downstream analysis (Table 1).

\section{2 smfATAC-Seq can be used to study chromatin accessibility of myofibers under different physiological}

113 conditions

114 In addition to adapting the ATAC-Seq method to study the chromatin accessibility of a single myofiber,

115 we also demonstrate the application of this technique for comparative analysis of chromatin accessibility

116 between myofibers under different conditions. For that purpose, we performed ATAC-Seq on myofibers

117 that were in a resting (uninjured) or regenerating (injured) state. Uninjured and injured (7 days post

118 cardiotoxin (CTX) induced injury) myofibers were isolated from wild type C57BL/6 mice and

119 smfATAC-Seq was performed to compare the changes in chromatin accessibility during regeneration. In

120 addition, as a further quality control we compared the chromatin accessibility between myonuclei within a

121 single myofiber and 5000 freshly isolated MuSCs. This analysis not only identified accessible regions of

122 chromatin in myofibers and MuSCs, but it also revealed a repertoire of active cis-regulatory elements in

123 each sample. 
Sahinyan et al. 2021 manuscript

124 Apart from the myofibers and their associated MuSCs, skeletal muscle also contains many non-myogenic

125 cells such as endothelial cells, adipocytes, hematopoietic cells, fibroblasts, fibro/adipogenic progenitors

126 (FAPs) and macrophages $(18,41,42)$. Our smfATAC-Seq method allows for the analysis of chromatin

127 accessibility of a single myofiber without the confounding effect of these contaminating cell types. Given

128 that the whole muscle contains non-myogenic cell types, we first compared smfATAC-Seq to an ATAC-

129 Seq performed on whole EDL muscle by Ramachandran, et al. 2019 (GSM3981673) (43) for the

130 enrichment of ATAC-Seq peaks on the genes of non-myogenic cells. We obtained the list of genes that

131 are solely expressed in the whole muscle (RPM of at least 10) but not in the myofibers (RPM of 0) by

132 using an RNA-Seq dataset performed on whole muscle and a single myofiber by Blackburn, et al. 2019

133 (GSE138591) (44). This list represents genes that are only expressed by the muscle resident non-

134 myogenic cell types, designated as non-fiber muscle genes. We determined the number of peaks in

135 smfATAC-Seq that overlap with non-fiber muscle genes which revealed that only $0.1 \%$ of the peaks

136 overlapped with the top 100 non-fiber muscle genes (Table 2). In comparison, $0.33 \%$ of the peaks in the

137 whole EDL muscle ATAC-Seq (GSM3981673) (43) overlapped with the top 100 non-fiber muscle genes

138 (Table 2). The significant difference in the overlap with the non-fiber muscle genes between the whole muscle ATAC-Seq and smfATAC-Seq suggest that the whole muscle ATAC-Seq has enrichment of 140 peaks associated with non-myogenic genes when compared to the smfATAC-Seq which implies that

141 smfATAC-Seq can successfully exclude the non-myogenic cell types. In contrast, number of overlapping

142 peaks with all the genes expressed in whole muscle in the EDL ATAC-Seq and smfATAC-Seq were

143 similar (Table 2). To further illustrate the absence of the non-myogenic cell types in the smfATAC-Seq

144 samples, peaks at the promoter regions of marker genes of muscle resident cells were searched for.

145 Specifically, Platelet and Endothelial Cell Adhesion Molecule 1 (Pecaml) was used to determine whether

146 endothelial cells were present (45). Similarly, Resistin (Retn) and Cd45 were used as markers for

147 adipocytes and hematopoietic cells, respectively (46,47). The cell Surface Antigen Thyl was the marker

148 selected for fibroblasts (48). In addition, Lymphocyte antigen 6a ( $L y 6 a)$ and Adhesion G protein-coupled

149 receptor E1 (Adgre1) were selected for fibro/adipogenic progenitors (FAPs) and macrophages,

150 respectively $(49,50)$. None of these marker genes had ATAC-Seq peaks at their promoters, indicating that

151 only a single myofiber is processed without any other contaminating cell types (Figure 2- Figure

152 Supplement 1).

153 smfATAC-Seq can be successfully applied to myofibers under different conditions. For instance, we 154 applied smf-ATAC-Seq to analyze the chromatin accessibility of myofibers under resting and CTX155 mediated injury conditions. In a disease condition or in injury, not all myofibers undergo damage or 156 regenerate to the same degree. Therefore, individual myofibers within a muscle can be in different 
Sahinyan et al. 2021 manuscript

157 physiological and disease conditions asynchronously (51). Damaged or regenerating myofibers can be

158 visualized by their characteristic feature of centrally located nuclei (52). Injured myofibers in this method

159 were visually selected by the presence of the centrally located myonuclei by Hoechst staining and the

160 selected myofiber was used for downstream processing with smfATAC-Seq (Figure 2A, 2B). The

161 selection of a specific myofiber that our smfATAC-Seq allows for, as well as the application of trypsin to

162 remove any associated cells that may be present, results in the sequencing of DNA fragments

163 corresponding purely to the myonuclei within a specific myofiber.

164 smfATAC-Seq can identify the accessible chromatin regions of a single myofiber

165 To validate the quality of the ATAC-Seq data generated from a single EDL myofiber, we first

166 investigated the profiles of the ATAC-seq samples from both injured and uninjured myofibers as well as

167 freshly sorted MuSCs. We investigated the similarity between biological replicates for each condition

168 (i.e., uninjured and injured myofibers and MuSCs) by visualization of ATAC-Seq peaks for the muscle-

169 specific gene muscle creatine kinase $(\mathrm{Ckm})(53)$, the housekeeping gene Gapdh and the MuSC specific

170 gene myogenic factor 5 (Myf 5) (54) (Figure 2- Figure Supplement 2A-C). This analysis not only

171 indicates that smfATAC-Seq can reliably detect chromatin accessibility in a single myofiber but also the

172 presence of comparable peaks in each biological replicate within the specific condition shows the

173 similarity between the samples (Figure 2- Figure Supplement 2A-C). In addition, Pearson correlation

174 analysis between the biological replicates showed a high correlation of ATAC-seq reads between the

175 replicates, indicating consistency within the samples (Figure 2- Figure Supplement 2D-J). Furthermore,

176 we mapped ATAC-Seq peaks with DNase-Seq from skeletal muscle (Sequence Read Archive, accession

177 \# SRX191047) and the similarity between our ATAC-Seq and previous DNase-Seq was confirmed

178 through the common peaks present for representative genes, as visualized on the IGV (Figure 2- Figure

179 Supplement 2A-C). We also analyzed the overlap between the smfATAC-Seq on EDL myofibers with the

180 ATAC-Seq performed on the whole EDL muscle by Ramachandran, et al. 2019 (GSM3981673) (43).

181 This analysis revealed that $65 \%$ of the smfATAC-Seq peaks in the uninjured myofibers overlap with the

182 whole EDL muscle ATAC-Seq (Table 3).

184 Accessible chromatin regions are associated with various histone marks such as H3K27ac and H3K4me3

185 (55-57). Thus, we compared the smfATAC-Seq to publicly available datasets of ChIP-Seq on H3K27ac

186 in EDL muscle that was previously performed by Ramachandran, et al. 2019 (GSM3515022,

187 GSM3515023) (43). The comparative analysis has revealed that there were only 97 peaks in the

188 smfATAC-Seq that did not overlap with the H3K27ac peaks, while the majority of the peaks, 6090 peaks,

189 were common to the H3K27ac peaks present in the entire EDL muscle (Figure 2- Figure supplement 2K ). 
Sahinyan et al. 2021 manuscript

190 This demonstrates that the accessible regions that are assessed by smfATAC-Seq correspond to the

191 regions of the chromatin marked by histones that are associated with open chromatin such as H3K27ac.

192 Overall, these analyses suggest that smfATAC-Seq can robustly measure chromatin accessibility and

193 identify active cis-regulatory elements in a single EDL myofiber.

194 Following the initial quality control and the correlation analysis, the biological replicates from the same 195 condition were pooled for further analysis. Peak annotation analysis for MuSCs revealed that more than

196 half of the peaks were in the intron/distal intergenic regions (i.e. enhancer regions) and about $25 \%$ of the 197 peaks were in the promoter region (Figure 2C, Table 4). Peak annotations for the uninjured and injured 198 single myofibers also showed a great proportion of peaks in the enhancer and promoter regions (Figure 199 2D, 2E, Table 4). In addition, an enrichment of ATAC-seq reads around Transcription Start Sites (TSS)

200 (+/- $1 \mathrm{~kb})$ from all datasets was observed, which is a typical result that is expected from ATAC-Seq (33) 201 (Figure 2F-H).

202 To further assess the quality of the ATAC-Seq data, we analyzed select genes that are expressed by either 203 MuSCs or myofibers. For instance, in the myofiber samples, we confirmed the presence of ATAC-seq 204 peaks in the promoter regions of $C k m$, Actin alpha 1 (Actal), Myogenic factor 6 (Myfo) and Myosin 205 heavy chain 4 (Myh4), all of which are expressed by myofibers but not MuSCs $(14,53,58,59)$ (Figure 2I206 L). On the other hand, in MuSCs we observed peaks in the promoter regions of Paired box 7 (Pax7), and 207 Myf5, genes that are known to be expressed in MuSCs (54,60) (Figure 2M-N). Gapdh was used a 208 housekeeping gene for all samples (Figure 2O) and Pou5f1, a marker of pluripotency, was used as a 209 negative control (Figure 2P). These observed peaks for known expressed genes demonstrate that our 210 method, smf-ATAC-Seq, can reliably analyse chromatin accessibility in a single myofiber.

211 Muscle regeneration and repair rely on the temporal expression of Myogenic Regulatory factors (MRFs), 212 Myf5, MyoD, Myog and Myf6/MRF4 (61-63). Therefore, we assessed the chromatin accessibility of the 213 MRFs in MuSCs and in the myofibers under homeostasis and regeneration (Figure 2- Figure Supplement

214 3). We observed peaks in the promoter regions of Myf5 only in the MuSCs but not in the myofibers and 215 peaks in the promoters of Myog and Myf6/MRF4 were solely observed in the myofibers (Figure 2- Figure 216 Supplement 3). However, we observed peaks in the promoter regions of MyoD in both the MuSCs and 217 myofibers (Figure 2- Figure Supplement 3). 
Sahinyan et al. 2021 manuscript

220 To show the global differences in chromatin accessibility between MuSCs, uninjured and injured

221 myofibers, we first performed heatmap clustering of Pearson correlation coefficients on all the

222 replicates/samples, which shows that the biological replicates within conditions are more similar to one

223 another than to those from the other conditions (Figure 3A). This can also be observed through Principal

224 Component Analysis (PCA) where each condition clusters separately, with the injured and uninjured

225 myofibers being more similar to one another than to MuSCs (Figure 3B). To test whether the differences

226 between regenerating and resting myofibers are overshadowed by their differences with MuSCs, we

227 performed heatmap clustering of Pearson correlation coefficients and PCA analysis for injured and

228 uninjured myofibers only, without MuSCs (Figure 3- Figure Supplement 1). This further highlighted how

229 the uninjured and injured myofibers cluster separately (Figure 3- Figure Supplement 1).

230 To ensure that the differences seen between myofibers were not due to differences in fiber types, we

231 investigated the chromatin accessibility of known marker genes for slow and fast fiber types. Troponin I2

232 (Tnni2) and Troponin T3 (Tnnt3), markers of fast fiber types, $(64,65)$ had a high level of chromatin

233 accessibility while Troponin T1 (Tnnt1) and Myosin heavy chain 7 (Myh7), which are expressed in slow

234 fiber types displayed no chromatin accessibility $(65,66)$ (Figure 3- Figure Supplement 2). This data

235 indicates that only fast fiber types were analyzed in this study and that the differences in the chromatin

236 state between the injured and uninjured myofibers were not due to the differences in the fiber types.

237 Differential analysis was performed on the ATAC-Seq peaks based on the regions defined by the

238 consensus peak sets derived from the uninjured and injured myofibers and MuSCs conditions. The

239 clustering analysis based on the consensus peak set shows the overall chromatin state differences between

240 the MuSCs and injured and unjured myofibers where the replicates within a condition are more similar to

241 one another than to those of the other conditions (Figure 3C).

242 The unique chromatin state in each condition can be observed through the pile up analysis that we have

243 performed for the peaks identified as more accessible $(\mathrm{LFC}>2)$ and less accessible $(\mathrm{LFC}<0.5)$ between

244 uninjured myofibers and MuSCs as well as between uninjured and injured myofibers (Figure 3D). In

245 addition, the proportion of the differential peaks corresponding to various genomic regions, such as

246 promoters and enhancers, differs depending on whether we compare MuSCs to myofibers or compare the

247 myofibers during regeneration and homeostasis. For instance, the differential peaks between uninjured

248 myofibers and MuSCs were mostly found close to the promoter region $(\leq 1 \mathrm{~kb})$ whereas in the uninjured

249 and injured myofibers comparison, a greater proportion of differential peaks were found in the

250 intron/distal intergenic regions (i.e. enhancer regions) (Figure 3E, Table 5). This implies that MuSCs and 
Sahinyan et al. 2021 manuscript

251 myofibers mostly differ in their promoter accessibility, whereas myofibers during homeostasis and

252 regeneration differ mostly at the level of distal regulatory elements.

253 Furthermore, we performed occupancy analysis (using DiffBind) in order to determine the unique and 254 common peaks between the conditions. Since the occupancy analysis relies on the peak score, the

255 distribution pattern of the peak scores for all the conditions was assessed and were found to be similar

256 despite the observed differences in the total number of peaks between the conditions (Figure 3- Figure

257 Supplement 3A). Occupancy analysis between uninjured myofibers and MuSCs revealed that MuSCs

258 have 45533 unique peaks while myofibers contain only 535 unique peaks which are not present in MuSCs

259 (Figure 3F). There are also many common accessible regions as seen from 5930 peaks that are common to

260 both uninjured myofibers and MuSCs. This analysis suggests that myonuclei share a large number of

261 open chromatin regions with their parental stem cells. On the other hand, the occupancy analysis between

262 uninjured and injured myofibers revealed that there are 6352 overlapping peaks between the regenerating

263 and resting myofibers. However, this analysis also revealed that there are 15352 unique peaks in the

264 injured myofibers and only 83 peaks that are unique to the resting myofiber (Figure 3F). Furthermore,

265 when comparing the read count between conditions around the center of unique peaks, it can be observed

266 that each condition displays a unique open chromatin signature (Figure 3- Figure Supplement 3C).

267 Comparative analysis of the chromatin state between MuSCs and myofibers

268 To get a better understanding of the functional differences in chromatin accessibility between MuSCs and 269 myofibers, we first performed Gene Ontology (GO Biological Process) analysis on the genes associated 270 with the nearest peaks from the uninjured myofiber and on the genes associated with the nearest unique 271 peaks in the myofiber compared to MuSCs. As expected, this revealed myofiber specific biological 272 processes such as myofiber structure and organization (Figure 4A and Figure 4- Figure Supplement 1A).

273 On the other hand, GO term analysis on all the genes nearest to each peak and on the genes associated

274 with the nearest unique peaks to MuSCs revealed biological processes such as adherens junction 275 organization, membrane permeability and regulation of notch signaling which play key roles in MuSCs 276 quiescence and function (67,68) (Figure 4B and Figure 4- Figure Supplement 1B). The analysis above

277 also revealed that genomic regions that remain in an open chromatin state when MuSCs fully differentiate

278 into myofibers correspond to genes that are involved in processes such as mitochondrial transport,

279 regulation of transcription and regulation of metabolites and energy (Figure 4- Figure Supplement 1C).

280 The changes in the chromatin state between MuSCs and myofibers can also be observed from the volcano

281 plots showing differential peaks between conditions labeled by their nearest gene (Figure 4D). For

282 instance, genomic regions associated with genes such as muscle-specific titin-capping protein (Tcap), a 
Sahinyan et al. 2021 manuscript

283

284

285

286

287

288

289

290

291

292

293

294

295

296

297

298

299

300

301

302

303

304

305

306

307

308

309

310

311

312

component of the skeletal muscle z-disc, as well as genes that are involved in regulatory and structural functions in skeletal muscle such as titin gene (Ttn) are associated with more accessible chromatin regions in the myofiber compared to MuSCs $(69,70)$ (Figure 4D).

Additionally, we performed GO term analysis between uninjured and injured myofibers, which revealed that globally, the accessible regions in the resting and regenerating myofibers corresponded to genes involved in similar processes. GO term analysis on the genes associated with the nearest peaks from uninjured myofibers and from the injured myofibers as well as the genes associated with the nearest peaks which are common between uninjured and injured myofibers revealed biological processes involved in striated muscle cell development, actomyosin structure, and sarcomere organization, which are important for myofiber structural formation and for the proper function of myofibers (Figure 4A, 4C, Figure 4Figure Supplement 1D). On the other hand, genes associated with the nearest unique peaks from the injured myofibers mostly belong to processes involved in structural components of the myofiber while the genes associated with the nearest unique peaks from uninjured myofibers correspond to genes involved in ion transport and metabolism (Figure 4- Figure Supplement 1E-F).

Furthermore, we analyzed the enrichment of transcription factor binding motifs in the sequences under peaks common between the injured and uninjured myofibers overlapping the promoters (+/- $5 \mathrm{~kb}$ of TSS)

(Figure 4- Figure Supplement 2A) as well as in the peaks that are unique to injured and uninjured myofibers overlapping the promoters (+/- 5kb of TSS) (Figure 4- Figure Supplement 2B). The top motifs that were enriched in the sequences under peaks common to injured and uninjured myofibers include binding site for Mef2a (Figure 4- Figure Supplement 2A). On the other hand, the top motifs that were enriched in the sequences under peaks unique to injured myofibers included binding sites for JUN and Stat3 (Figure 4- Figure Supplement 2B). However, due to the low number of unique peaks in the uninjured myofibers (Figure 3F), there was no significant motif that enriched in that peak set.

\section{Identification of cell type specific pathways by global analysis of chromatin accessibility}

To further understand the functional differences in chromatin accessibility between different cell types, we investigated the cell-type-specific pathways. To accomplish this, Gene Set Enrichment Analysis (GSEA) was performed on genes associated with differentially accessible peaks between the conditions. Importantly, the GSEA between uninjured and injured myofibers revealed that inflammatory response and I12-Stat5 signaling, and injury related pathways are still operational even after seven days of CTXmediated injury to muscle (71) (Figure 5A). 
Sahinyan et al. 2021 manuscript

313 The GSEA between uninjured myofibers and MuSCs revealed that one of the significantly enriched

314 pathways is myogenesis, where we can observe that genes associated with differentiation and myofiber

315 function such as $M y f 6, C \mathrm{~km}$ and Tropomyosin 2 (Tpm2) (14,53,72,73) have higher accessibility in the

316 myofiber compared to MuSCs (Figure 5B-5D). On the other hand, genes associated with quiescence and

317 MuSCs such as alpha 7 integrin (Itga7) and Gpx3 are more accessible in MuSCs compared to the

318 myofiber, as expected (74,75) (Figure 5C, 5E).

Moreover, differential chromatin accessibility between the MuSCs and their myofiber derivatives show differences in pathways that are known to be important for muscle, such as Notch and TGF $\beta$ signalling $(67,68,76,77)$ (Figure 5- Figure Supplement 1). For example, increased accessibility of Notchl is seen in MuSCs while increased accessibility of Jagged-2 (Jag2) is observed in the myofibers regardless of whether they are regenerating or homeostatic as seen by the height of the peaks at their promoters (Figure 5- Figure Supplement 1A-C). On the other hand, for TGF $\beta$ signalling, Noggin (Nog) shows more accessibility in the myofibers while bone morphogenetic protein-4 (Bmp4) has increased accessibility in MuSCs (Figure 5- Figure Supplement 1D-F). Taken together, this data shows that smfATAC-Seq is an effective method to analyze chromatin accessibility and to identify active cis-regulatory elements in a single muscle fiber as well as to compare muscle fibers under different physiological conditions.

\section{Comparative analysis of the chromatin state between WT and MDX myofibers}

332 To demonstrate the applicability of our method to a disease condition, we performed smfATAC-Seq on 333 myofibers isolated from mdx mice, a model for Duchenne's muscular dystrophy (DMD), and their wild 334 type (WT) C57BL/10ScSn counterparts. In order to solely assess the effect of the loss of dystrophin 335 without the effect of regeneration, myofibers that were not actively regenerating were selected for 336 processing from both conditions. As was performed in the previous cohort, we began by confirming the 337 similarity between biological replicates by visualization of ATAC-Seq peaks for $\mathrm{Ckm}$ and housekeeping 338 gene Rps2, as well by the Pearson correlation analysis between the biological replicates (Figure 6- Figure

339 Supplement 1). After consistency of the samples within the conditions was established, the biological

340 replicates from the same condition were pooled for further analysis. First, enrichment of the ATAC-Seq 341 reads around the TSS from both mdx and WT myofibers was confirmed (Figure 6A). Peak annotation 342 analysis revealed that most of the peaks were in the promoter and enhancer regions for both data sets 343 (Figure 6B, Table 4). To further show that smf-ATAC-Seq can successfully assess the chromatin 344 accessibility in a single myofiber of an mdx and WT EDL muscle, we looked at the presence of ATAC345 seq peaks in the promoter regions of Ckm, Actal, Myh4, and the housekeeping genes Gapdh and Rps2, 
Sahinyan et al. 2021 manuscript

whereas Pou5fl was used as a negative control (Figure 6- Figure Supplement 2A-F). We also confirmed that the myofibers from mdx and WT conditions were fast type (Figure 6- Figure Supplement 2G-H) and that they exclusively represent the myonuclei, without the presence of confounding cell types in the muscle (Figure 6- Figure Supplement 2I-N).

To investigate the differences in the chromatin state between $\mathrm{mdx}$ and WT, we first performed heatmap clustering of Pearson correlation coefficients and PCA (Figure 6C, 6D). These analyses showed that, mdx and WT myofibers cluster separately and the biological replicates for each condition generally cluster together (Figure 6C,6D). We then performed differential analysis of ATAC-Seq peaks followed by pileup analysis for the less accessible (LFC < 0.5) and more accessible (LFC> 2) peaks between WT and mdx myofibers (Figure 6E). The results clearly showed that mdx and WT myofibers exhibit extensive differences in their chromatin states (Figure 6E). Peak annotation analysis on the differential peaks between mdx and WT myofibers revealed that more than half of the differential peaks were found in enhancer regions, indicating that they differ mostly at the level of distal regulatory elements (Figure 6F, Table 5). In addition, occupancy analysis revealed that there are 22,967 overlapping peaks between mdx and WT myofibers, and that mdx myofibers possess 7599 unique peaks, while WT myofibers contain 2895 unique peaks (Figure 6G).

Furthermore, to understand the functional differences in chromatin accessibility, we performed Gene Ontology (GO Biological Process) analysis on the genes associated with the nearest peak for all peaks in the mdx and WT myofibers, as well as on the genes associated with the nearest common peaks between them (Figure 6- Figure Supplement 3). These analyses revealed processes involved in mitochondrial transport, myofibril assembly, sarcomere organization and striated muscle cell development which are important for myofiber structure, organization, and function (Figure 6- Figure Supplement 3). However, GO term analysis on the unique peaks of each condition revealed that different processes are affected between mdx and WT. GO term analysis on the genes associated with the nearest unique peaks from mdx myofibers revealed processes that are important for myofiber structure and organization such as actin cytoskeleton organization and striated muscle cell differentiation (Figure 6H). On the other hand, GO term analysis on the genes associated with the nearest unique peaks from WT myofibers revealed processes mostly involved in metabolism (Figure 6I). Since the observed differential biological processes between WT and mdx myofibers were similar to those seen between injured vs uninjured myofibers, we then compared the overall differences in chromatin accessibility between mdx, WT, and injured myofibers. We performed heatmap clustering of Pearson correlation and PCA analysis between WT, mdx and injured myofibers (Figure 6- Figure Supplement 4). These analyses have revealed that injured myofibers were more similar to the mdx than they are to the WT C57BL/10ScSn myofibers. However, as 
Sahinyan et al. 2021 manuscript

expected due to the different backgrounds of the mice between injured and the WT and mdx mice, WT and mdx myofibers were more similar to each other than they are to the injured C57BL/6 myofibers (Figure 6- Figure Supplement 4).

Finally, we determined the top motifs that are enriched in the sequences under the peaks that are common between the mdx and WT myofibers overlapping the promoters (+/- $5 \mathrm{~kb}$ of TSS) (Figure 6- Figure Supplement 5A) as well as the sequences under peaks that are unique to mdx and WT overlapping the promoters (+/- $5 \mathrm{~kb}$ of TSS) (Figure 6- Figure Supplement 5B-C). The top significantly enriched motifs in the peaks common between mdx and WT included Mef2a and JUN (Figure 6- Figure Supplement 5A) while the top motifs enriched in the peaks unique to $\mathrm{mdx}$ included transcription factors such as Foxo1 (Figure 6- Figure Supplement 5B).

Overall, this data shows that smfATAC-Seq can be reliably used to study myofibers in disease conditions, revealing that there are substantial differences in the chromatin accessibility of myofibers in the mdx mice compared to their WT counterparts.

\section{DISCUSSION}

Analysis of the myofiber-specific chromatin state and gene expression profile is very limited due to the heterogenous nature of muscle with the presence of numerous non-myogenic cells in the tissue. Whole muscle or muscle biopsies represents a pooled result of numerous cell types which are present in the muscle tissue. To overcome this limitation, single nucleus RNA-Seq (snRNA-Seq) and ATAC-Seq (snATAC-Seq) have been developed and performed on myonuclei to allow for the computational removal of other cell types (19-21). However, these methods still sequence all myonuclei present in the muscle and cannot distinguish between different myofibers within a muscle. Although snATAC-Seq provides high dimensionality, it is limited in sequencing depth due to the generation of sparse reads. Although computational pseudo bulking of snATAC-Seq can increase read numbers for comparative analysis between samples and conditions, the pooled reads represent the average of all myonuclei within the sample.

In this study we have introduced a highly effective protocol based on adaption of OMNI ATAC-Seq (30) to quantify chromatin accessibility of a single EDL myofiber with high resolution and sequencing depth. This method allows for comparative analysis of chromatin accessibility within and between muscle types with a potential for wide-spread use in future studies to investigate myofiber-specific epigenetic alterations in skeletal muscle. 
Sahinyan et al. 2021 manuscript

413 The smfATAC-Seq protocol that we introduce in this study investigates the open chromatin state of

414 myofibers at a single myofiber resolution, and with a high sequencing depth that allows for peak calling

415 and differential peak analysis. Using this method, we have demonstrated that accessible chromatin

416 regions of myonuclei contained within a single EDL myofiber can be tagmented and that high-quality

417 sequencing ready libraries can be generated from these fragments. Sequencing of these libraries allow for

418 sufficient depth and peak calling that can be used for genome-wide analysis of chromatin accessibility

419 between myofibers. In this study, we have also demonstrated that smfATAC-Seq strictly investigates a

420 single myofiber without the confounding presence of muscle resident non-myogenic cell types.

421 Additionally, application of trypsin to the isolated myofibers effectively removes MuSCs that are

422 associated with myofibers (44) and was confirmed by the absence of peaks at the promoters of known

423 genes associated with muscle stem and niche cells. Although all of the smfATAC-Seq samples sequenced

424 in this study were fast type myofibers, this protocol can be used to distinguish between different fiber

425 types which could be applied to study myofiber heterogeneity under various physiological conditions.

426 smfATAC-Seq peaks associated with genes involved in muscle structure and function such as Actal, Ckm

427 and the myosin heavy chain cluster, indicates that our smfATAC-Seq is a robust technique to investigate

428 genome-wide chromatin accessibility of a single myofiber.

430 A key implication of this technique is its applicability to the study of changes in chromatin accessibility

431 between myofibers in different contexts. As a demonstration of this, we have performed smfATAC-Seq

432 on uninjured myofibers as well as injured myofibers isolated seven days post-injury to investigate the

433 changes in chromatin accessibility that occurs during regeneration. Through Pearson correlations and

434 PCA analysis, we showed resting and injured myofibers cluster separately, indicating the power of

435 smfATAC-Seq to determine chromatin signature from even the minute starting material of a single

436 myofiber. In addition, through occupancy analysis, we showed that there is a large difference in the

437 number of unique peaks present in the injured myofibers compared to uninjured myofibers. This indicates

438 that there are major modifications to chromatin accessibility in the context of regeneration. However, GO

439 term analysis of genes associated with the accessible chromatin regions in both injured and uninjured

440 fibers are very similar in the biological processes and pathways such as striated muscle cell development,

441 actomyosin structure and sarcomere organization, which are key factors for the proper structure and

442 function of muscle, are enriched. Despite these similarities, there are certain trends in which uninjured

443 myofibers have increased accessibility in genes involved in energy metabolism, while injured myofibers

444 have greater accessibility in genes involved in myogenesis and inflammatory response which is what

445 would be expected in the case of an injury and regeneration (78). Despite the increase in chromatin 
Sahinyan et al. 2021 manuscript

accessibility during injury, the accessible chromatin regions in both injured and uninjured fibers are associated with genes involved in similar biological processes. This similarity at the gene network despite differences in chromatin profile may suggest activation of multiple enhancers on core muscle structural genes in the case of injury. Another possible reason could be the length of the recovery time where at seven days post injury, a number of genes activated early in the regeneration process may have returned to levels seen in the steady state. Previously, a study investigating the changes in the transcriptional profile of MuSCs and various muscle resident cells throughout different time points of muscle injury using single cell RNA-Seq, revealed that after seven days of regeneration most cell types returned to a state that was similar to homeostasis (41). Therefore, it is possible that harvesting the injured EDL myofibers seven days post injury allowed these myofibers to return to a state reminiscent of homeostatic myofibers. Further, our analyses of the myofibers in this study indicates that this technique can effectively compare samples between conditions and could see future use in the study of chromatin accessibility of myofibers under different biologically relevant conditions.

We have also used smfATAC-Seq to compare changes in chromatin accessibility between MuSCs and myofibers. Our data shows that the regions of open chromatin in the myofibers correspond to genes involved in structural components of the muscle, such as the z-disc, which are important for the proper functioning of the muscle. On the other hand, open regions of chromatin in the MuSCs mostly correspond to genes involved in membrane permeability, adherens junction organization and signalling pathways implicated in the regulation of MuSC function (79). The analysis also revealed that chromatin regions that are accessible in both MuSCs and myofibers correspond to genes that are crucial for the general function of cells such as those involved in mitochondrial transport, regulation of transcription and regulation of metabolites and energy.

Lastly, we performed smfATAC-Seq on non-regenerating myofibers isolated from the mouse model of DMD (40) and their WT counterparts. DMD is a type of muscular dystrophy caused by a loss of function mutation in the Dystrophin $(D M D)$ gene that encodes for a protein that has a crucial role in muscle structure (80). Lack of functional dystrophin in DMD leads to unstable and fragile myofibers that continuously need to be regenerated, in turn leading to progressive muscle degeneration (80). We have not only shown that smfATAC-Seq can reliably investigate the chromatin accessibility from a single myofiber of mdx and WT EDL muscle, but through Pearson correlations, PCA and differential peak analysis we have also shown that DMD is associated with substantial alterations in chromatin accessibility in myonuclei. Through occupancy and GO term analyses, we have shown that a great proportion of peaks that are common between the mdx and WT myofibers are mostly associated with 
Sahinyan et al. 2021 manuscript

processes involved in muscle structure and organization. However, we have shown that mdx myofibers have more unique peaks compared to the WT, suggesting that chromatin accessibility of myonuclei is increased in the mdx disease model. Our analyses have shown that the unique peaks in mdx are associated with biological processes involved in myofiber structure and organization. On the other hand, unique peaks in the WT myofibers are associated with processes mostly involved in energy and metabolism. It is possible that the progressive muscle degeneration due to loss of muscle fiber integrity and stability causes mdx myofibers to compensate by increasing the activity of processes involved in muscle structure and organization while WT myofibers retain their activity in metabolism. It should be noted that the differences in chromatin accessibility that we have observed between resting and regenerating myofibers and between the mdx and WT myofibers are similar, which could be explained by the degeneration and continuous round of regeneration in the mdx myofibers.

Studies in the future could utilize smfATAC-Seq to further investigate the changes in chromatin accessibility in the mdx myofibers and investigate the changes associated with DMD at the level of chromatin to potentially investigate therapeutic avenues for this disease, as well as other muscle wasting diseases.

Overall, smfATAC-Seq is a robust molecular tool that can be used to analyze genome-wide chromatin accessibility of a single myofiber. The sequencing depth from this approach, allows for in-depth analysis, peak calling, quantitative analysis of chromatin accessibility and to identify active enhancers and promoters in a single muscle fiber. smfATAC-Seq can be used to study the epigenetic alterations that occur in muscle fibers during development, diseases, and in response to exercise.

(1)

(1) 
Sahinyan et al. 2021 manuscript

\section{Tables}

515

516 Table 1: Sequencing read information for smfATAC-Seq and MuSCs ATAC-Seq libraries

\begin{tabular}{|c|c|c|c|c|c|c|c|c|c|}
\hline Library & $\begin{array}{l}\text { Number } \\
\text { of Raw } \\
\text { Reads }\end{array}$ & $\begin{array}{l}\text { Number } \\
\text { of } \\
\text { Surviving } \\
\text { Reads }\end{array}$ & $\begin{array}{l}\text { Aligned } \\
\text { Filtered } \\
\text { Reads } \\
\text { (mm10 } \\
\text { reference } \\
\text { ) }\end{array}$ & $\begin{array}{l}\text { Duplicat } \\
\text { e Reads }\end{array}$ & $\begin{array}{l}\text { Mitochondri } \\
\text { al Reads }\end{array}$ & $\begin{array}{l}\text { Percentage } \\
\text { of } \\
\text { Mitochondri } \\
\text { al Reads } \\
(\%)\end{array}$ & $\begin{array}{l}\text { Final } \\
\text { Reads } \\
\text { Aligned }\end{array}$ & $\begin{array}{l}\text { Numb } \\
\text { er of } \\
\text { Peaks }\end{array}$ & $\begin{array}{l}\text { Fractio } \\
\mathrm{n} \text { in } \\
\text { peaks } \\
\text { (FrIP) }\end{array}$ \\
\hline $\begin{array}{l}\text { Muscle } \\
\text { Stem } \\
\text { Cells_1 }\end{array}$ & $\begin{array}{l}1759247 \\
34\end{array}$ & $\begin{array}{l}1139384 \\
36\end{array}$ & $\begin{array}{l}1031301 \\
86\end{array}$ & $\begin{array}{l}4762383 \\
6\end{array}$ & 529967 & 0.51 & $\begin{array}{l}5497638 \\
3\end{array}$ & 65568 & 0.3642 \\
\hline $\begin{array}{l}\text { Muscle } \\
\text { Stem } \\
\text { Cells_2 }\end{array}$ & $\begin{array}{l}1749659 \\
36\end{array}$ & $\begin{array}{l}1173572 \\
12\end{array}$ & $\begin{array}{l}1035700 \\
09\end{array}$ & $\begin{array}{l}4367248 \\
4\end{array}$ & 374176 & 0.36 & $\begin{array}{l}5952334 \\
9\end{array}$ & 68658 & 0.1971 \\
\hline $\begin{array}{l}\text { Muscle } \\
\text { Stem } \\
\text { Cells_3 }\end{array}$ & $\begin{array}{l}1319903 \\
80\end{array}$ & $\begin{array}{l}9126158 \\
4\end{array}$ & $\begin{array}{l}7994412 \\
1\end{array}$ & $\begin{array}{l}3129945 \\
6\end{array}$ & 223540 & 0.28 & $\begin{array}{l}4842112 \\
5\end{array}$ & 69573 & 0.1296 \\
\hline Injured_1 & $\begin{array}{l}2299354 \\
26\end{array}$ & $\begin{array}{l}1172126 \\
78\end{array}$ & $\begin{array}{l}9004000 \\
2\end{array}$ & $\begin{array}{l}8102492 \\
6\end{array}$ & 830215 & 0.92 & 8184861 & 32853 & 0.2885 \\
\hline Injured_2 & $\begin{array}{l}1945638 \\
70\end{array}$ & $\begin{array}{l}1299349 \\
72\end{array}$ & $\begin{array}{l}9875215 \\
7\end{array}$ & $\begin{array}{l}8854932 \\
9\end{array}$ & 1300615 & 1.32 & 8902213 & 28351 & 0.2863 \\
\hline Injured_3 & $\begin{array}{l}1424115 \\
36\end{array}$ & $\begin{array}{l}6288855 \\
2 \\
\end{array}$ & $\begin{array}{l}5213245 \\
5\end{array}$ & $\begin{array}{l}4227107 \\
9\end{array}$ & 868808 & 1.67 & 8992568 & 25002 & 0.2325 \\
\hline $\begin{array}{l}\text { Uninjured } \\
-1\end{array}$ & $\begin{array}{l}1454654 \\
10\end{array}$ & $\begin{array}{l}7578145 \\
6\end{array}$ & $\begin{array}{l}6103456 \\
9\end{array}$ & $\begin{array}{l}5258831 \\
5\end{array}$ & 1274332 & 2.09 & 7171922 & 12276 & 0.2181 \\
\hline $\begin{array}{l}\text { Uninjured } \\
2\end{array}$ & $\begin{array}{l}1510158 \\
52\end{array}$ & $\begin{array}{l}6419270 \\
6\end{array}$ & $\begin{array}{l}5012028 \\
2\end{array}$ & $\begin{array}{l}4591484 \\
1\end{array}$ & 965037 & 1.93 & 3240404 & 14742 & 0.3208 \\
\hline MDX_1 & $\begin{array}{l}1075407 \\
62\end{array}$ & $\begin{array}{l}5097973 \\
2\end{array}$ & $\begin{array}{l}4048580 \\
3\end{array}$ & $\begin{array}{l}3620590 \\
8\end{array}$ & 802561 & 1.98 & 3477334 & 40833 & 0.7256 \\
\hline MDX_2 & $\begin{array}{l}1031307 \\
26\end{array}$ & $\begin{array}{l}5420972 \\
2\end{array}$ & $\begin{array}{l}4645547 \\
2\end{array}$ & $\begin{array}{l}3729153 \\
1\end{array}$ & 1099747 & 2.37 & 8064194 & 39254 & 0.4932 \\
\hline MDX_3 & $\begin{array}{l}1081306 \\
62\end{array}$ & $\begin{array}{l}4892090 \\
4\end{array}$ & $\begin{array}{l}4067735 \\
9\end{array}$ & $\begin{array}{l}3448400 \\
3\end{array}$ & 1171316 & 2.88 & 5022040 & 35691 & 0.5589 \\
\hline WT_1 & $\begin{array}{l}1042195 \\
78\end{array}$ & $\begin{array}{l}4391490 \\
2\end{array}$ & $\begin{array}{l}3416214 \\
2\end{array}$ & $\begin{array}{l}2860049 \\
8\end{array}$ & 1651199 & 4.83 & 3910445 & 26873 & 0.7283 \\
\hline WT_2 & $\begin{array}{l}1101086 \\
92\end{array}$ & $\begin{array}{l}3741193 \\
6\end{array}$ & $\begin{array}{l}3129922 \\
2\end{array}$ & $\begin{array}{l}2534532 \\
1\end{array}$ & 1143317 & 3.65 & 4810584 & 28430 & 0.64 \\
\hline WT_3 & $\begin{array}{l}1835835 \\
06\end{array}$ & $\begin{array}{l}7248935 \\
4\end{array}$ & $\begin{array}{l}5698363 \\
7 \\
\end{array}$ & $\begin{array}{l}4931092 \\
3\end{array}$ & 1840265 & 3.23 & 5832449 & 39178 & 0.7611 \\
\hline WT_4 & $\begin{array}{l}8653384 \\
0\end{array}$ & $\begin{array}{l}3670870 \\
6\end{array}$ & $\begin{array}{l}2871489 \\
3\end{array}$ & $\begin{array}{l}2515796 \\
5\end{array}$ & 1404712 & 4.89 & 2152216 & 21252 & 0.752 \\
\hline
\end{tabular}


Sahinyan et al. 2021 manuscript

522

523

524

525

526

527

528

529

530

531

532

533 
Sahinyan et al. 2021 manuscript

*Whole EDL muscle ATAC-Seq was retrieved from "Dynamic enhancers control skeletal muscle identity and reprogramming, PLoS Biology, Ramachandran, et al. 2019." This data is accessible through the GEO accession number GSM3981673.

Table 4: Percentage of total peaks found in each genomic feature

\begin{tabular}{|l|l|l|l|l|l|}
\hline & $\begin{array}{l}\text { Muscle Stem } \\
\text { Cells }(\%)\end{array}$ & $\begin{array}{l}\text { Injured } \\
\text { Myofiber }(\%\end{array}$ & $\begin{array}{l}\text { Uninjured } \\
\text { Myofiber (\%) }\end{array}$ & $\begin{array}{l}\text { MDX } \\
\text { Myofiber (\%) }\end{array}$ & WT Myofiber (\%) \\
\hline Promoter (+/- 1kb TSS) & 20.66 & 31.61 & 56.54 & 35.58 & 35.15 \\
\hline $\begin{array}{l}\text { Promoter (+/- 1kb } \\
\text { and/or +/- 2kb TSS) }\end{array}$ & 4.81 & 4.84 & 3.45 & 3.78 & 4.53 \\
\hline $\begin{array}{l}\text { Promoter ((+/- 2kb } \\
\text { and/or +/- 3kb TSS) }\end{array}$ & 4.37 & 3.92 & 3.01 & 4.14 & 4.30 \\
\hline 5'UTR & 0.34 & 0.27 & 0.23 & 0.46 & 0.39 \\
\hline 3'UTR & 2.50 & 1.82 & 1.15 & 2.86 & 2.58 \\
\hline 1 st Exon & 1.83 & 1.47 & 1.53 & 1.94 & 1.78 \\
\hline Other Exon & 4.75 & 3.42 & 2.19 & 4.74 & 4.25 \\
\hline 1 st Intron & 11.85 & 10.87 & 7.35 & 10.93 & 10.56 \\
\hline Other Intron & 20.80 & 18.84 & 10.35 & 18.81 & 18.30 \\
\hline Downstream $(<=300 \mathrm{~kb})$ & 1.16 & 1.01 & 0.69 & 1.02 & 0.99 \\
\hline Distal Intergenic & 26.95 & 21.93 & 13.51 & 15.74 & 17.15 \\
\hline
\end{tabular}

Table 3: Percentage of overlapping peaks between smfATAC-Seq from uninjured myofibers and whole EDL muscle ATAC-Seq

\begin{tabular}{|l|r|}
\hline & \multicolumn{1}{|c|}{$\begin{array}{c}\text { Percent } \\
\text { Overlap (\%) }\end{array}$} \\
\hline $\begin{array}{l}\text { smfATAC-Seq peaks that overlap } \\
\text { with EDL-ATAC-Seq by at least 1 } \\
\text { bp }\end{array}$ & 65.9510759 \\
\hline $\begin{array}{l}\text { smfATAC-Seq peaks that overlap } \\
\text { with EDL-ATAC-Seq by at least } \\
20 \%\end{array}$ & 61.4951279 \\
\hline $\begin{array}{l}\text { smfATAC-Seq peaks that overlap } \\
\text { with EDL-ATAC-Seq by at least } \\
\text { 40\% }\end{array}$ & 52.6136825 \\
\hline $\begin{array}{l}\text { smfATAC-Seq peaks that overlap } \\
\text { with EDL-ATAC-Seq by at least } \\
60 \%\end{array}$ & 42.1082014 \\
\hline $\begin{array}{l}\text { smfATAC-Seq peaks that overlap } \\
\text { with EDL-ATAC-Seq by at least } \\
\text { 90\% }\end{array}$ & 24.3453106 \\
\hline
\end{tabular}


Sahinyan et al. 2021 manuscript

Table 5: Percentage of differential peaks in each genomic feature

\begin{tabular}{|l|l|l|l|}
\hline & $\begin{array}{l}\text { Uninjured Myofiber } \\
\text { vs. MuSCs (\%) }\end{array}$ & $\begin{array}{l}\text { Uninjured vs Injured } \\
\text { Myofiber (\%) }\end{array}$ & WT vs MDX Myofiber (\%) \\
\hline Promoter (+/- 1kb TSS) & 43.07 & 25 & 29.92 \\
\hline $\begin{array}{l}\text { Promoter (+/- 1kb and/or +/- 2kb } \\
\text { TSS) }\end{array}$ & 3.36 & 7.81 & 3.68 \\
\hline $\begin{array}{l}\text { Promoter (+/- 2kb and/or +/- 3kb } \\
\text { TSS) }\end{array}$ & 3.39 & 3.12 & 4.49 \\
\hline 5'UTR & 0.37 & 0.78 & 0.46 \\
\hline 3'UTR & 1.95 & 3.12 & 2.99 \\
\hline 1 st Exon & 2.29 & 3.91 & 1.84 \\
\hline Other Exon & 3.85 & 7.81 & 4.49 \\
\hline 1 st Intron & 9.16 & 13.28 & 14.84 \\
\hline Other Intron & 13.34 & 18.75 & 24.86 \\
\hline Downstream $(<=300 \mathrm{~kb})$ & 0.83 & 0.78 & 0.12 \\
\hline Distal Intergenic & 18.39 & 15.62 & 12.31 \\
\hline & & & \\
\hline
\end{tabular}

\section{Figure Legends}

\section{Figure 1: Schematic of ATAC-seq performed on a single myofiber}

Schematic of the steps and reactions involved in the preparation of sequencing ready libraries of single myofiber DNA for ATAC-Seq. Briefly, myofibers were isolated from the EDL muscle and an individual myofiber was transferred to a $0.2 \mathrm{~mL}$ microtube. The myofiber was then lysed with $\mathrm{ddH}_{2} \mathrm{O}$ and the myonuclei were permeabilized with $0.5 \%$ Triton X-100. Then, open chromatin regions were tagmented with hyperactive Tn5 transposase and the DNA fragments were purified through column purification. The tagmented DNA was then amplified by PCR and Nextera adapters were incorporated. Finally, size selection and purification were performed using $0.85 \mathrm{X}$ AMPure beads, resulting in sequencing ready libraries. Figure was made using BioRender

\section{Figure 2: smfATAC-Seq can effectively identify the accessible regions on a single myofiber}

(A) Representative picture of an isolated WT C57BL/6J uninjured myofiber stained for DAPI showing the presence and location of myonuclei. Scale bar $=50 \mu \mathrm{m}$. (B) Representative picture of an isolated WT C57BL/6J injured myofiber (7 days post cardiotoxin induced injury) stained for DAPI showing centrally located myonuclei as a marker of a regenerating fiber. Scale bar $=50 \mu \mathrm{m}$. (C-E) Peak annotation pie charts for ATAC-Seq peaks of MuSCs, injured myofibers and uninjured myofibers, respectively. (F-H) 
Sahinyan et al. 2021 manuscript

615 Heatmaps showing enrichment at transcription start site (TSS) for the ATAC-Seq libraries of MuSCs,

616 injured myofibers and uninjured myofibers, respectively. (I-P) IGV snapshots of known genes expressed

617 in muscle fiber and/or MuSCs displaying accessibility on their respective TSS. (I) The muscle creatine

618 kinase $(\mathrm{Ckm})$. (J) Actin alpha 1 (Actal). (K) Part of the myosin heavy chain (Myh) gene cluster. (L)

619 Myogenic factor 6 (Myfo). (M) Paired box 7 (Pax7). (N) Myogenic factor 5 (Myf5). (O) Housekeeping

620 gene Gapdh. (P) POU Class 5 homeobox 1 (Pou5f1) as a negative control.

621 *ATAC-Seq was performed in biological replicates; ( $n=3$ MuSCs, $n=3$ injured myofibers, $n=2$ uninjured 622 myofibers)

624 Figure 3: Uninjured and injured myofibers and MuSCs display distinct chromatin states

625 (A)Heatmap clustering of Pearson correlation coefficients showing the correlation between the replicates

626 of the conditions in the regions defined by the union peakset (merged peaks of all replicates/samples). (B)

627 Projection of samples along the first two principal components found by PCA showing the separate

628 clustering of different samples and the clustering of each replicate of the same condition together. (C)

629 Heatmap clustering of Pearson correlation coefficients indicating the correlation between the replicates in

630 the regions defined by the consensus peakset derived from the uninjured myofibers, injured myofibers

631 and MuSCs. (D) Pile up analysis of differentially accessible peaks between uninjured myofibers and

632 MuSCs and between injured myofibers and uninjured myofibers. Less accessible regions: FDR $<0.05$

633 and LFC < 0.5. More accessible peaks: FDR < 0.05 and LFC >2. (E) Peak annotation pie charts for the

634 differentially accessible peaks between uninjured myofibers vs MuSCs and uninjured myofibers vs

635 injured myofibers. (F) Venn diagram of the number of ATAC-Seq peaks that are unique or overlapping

636 between uninjured myofibers vs MuSCs and uninjured myofibers vs injured myofibers.

637 *ATAC-Seq was performed in biological replicates; ( $n=3$ MuSCs, $n=3$ injured myofibers, $n=2$ uninjured 638 myofibers)

640 Figure 4: Comparative analysis of chromatin state between uninjured myofibers and MuSCs and 641 between uninjured myofibers and injured myofibers

642 (A-C) Gene Ontology (GO Biological Process) analysis of genes associated with ATAC-Seq peaks based 643 on association by proximity using Genomic Regions Enrichment of Annotations Tool (GREAT) (81) for

644 all peaks present in the uninjured myofibers, MuSCs and injured myofibers respectively. (D) Volcano

645 plot of differentially accessible regions/peaks identified by FDR $<0.05$ and LFC $>=1$ between uninjured

646 myofibers and MuSCs. Each dot represents a differentially accessible region/peak and the distance to the

647 nearest gene is annotated. (E) Volcano plot of differentially accessible regions/peaks identified by FDR < 
Sahinyan et al. 2021 manuscript

6480.05 and LFC >= 1 between uninjured myofibers and injured myofibers. Each coloured dot represents a

649 differentially accessible region/peak and the distance to the nearest gene is annotated.

650 *ATAC-Seq was performed in biological replicates; ( $n=3$ MuSCs, $n=3$ injured myofibers, $n=2$ uninjured 651 myofibers)

652

653 Figure 5: Identification of cell type specific pathways by global analysis of chromatin accessibility

654 (A) Gene Set Enrichment Analysis performed on genes nearest to the differentially accessible

655 regions/peaks for uninjured myofibers compared to injured myofibers. Top 10 enriched pathways are

656 shown although do not reach significance. (B) Gene Set Enrichment Analysis performed on genes nearest

657 to the differentially accessible regions/peaks for uninjured fibers compared to MuSCs. Top 10

658 significantly enriched pathways are shown (FDR < 0.01). (C) Heatmap for genes involved in myogenesis

659 based on read counts of MuSCs and uninjured fibers $+/-1 \mathrm{~kb}$ of the TSS of each gene in the myogenic

660 pathway. (D) IGV snapshot of Tropomyosin 2 (Tpm2). (E) IGV snapshot of Glutathione Peroxidase 3

661 (Gpx3).

662 *ATAC-Seq was performed in biological replicates; ( $n=3$ MuSCs, $n=3$ injured myofibers, $n=2$ uninjured 663 myofibers)

664

665 Figure 6: Comparative analysis of chromatin state between MDX and WT myofibers

666 (A) Heatmaps showing enrichment at transcription start site (TSS) for the ATAC-Seq libraries of MDX 667 and WT myofibers respectively. (B) Peak annotation pie charts for ATAC-Seq peaks of MDX and WT

668 myofibers respectively. (C) Heatmap clustering of Pearson correlation coefficients showing the 669 correlation between the replicates of the conditions in the regions defined by the union peakset (merged 670 peaks of all replicates/samples). (D) Projection of samples along first two principal components found by 671 PCA showing the separate clustering of different samples and the clustering of each replicate of the same 672 condition together. (E) Pile up analysis of differentially accessible peaks between WT and MDX

673 myofibers. Less accessible regions: FDR $<0.05$ and LFC $<0.5$. More accessible peaks: FDR $<0.05$ and 674 LFC >2. (F) Peak annotation pie charts for the differentially accessible peaks between WT and MDX 675 myofibers. (G) Venn diagram of the number of ATAC-Seq peaks that are unique or overlapping between 676 WT and MDX myofibers. (H) Gene Ontology (GO Biological Process) analysis of genes associated with 677 unique peaks present in the MDX myofiber compared to WT myofibers, based on the proximity of the 
Sahinyan et al. 2021 manuscript

678 peaks to the genes. (I) Gene Ontology (GO Biological Process) analysis of genes associated with unique

679 peaks present in the WT myofiber compared to MDX.

680 *ATAC-Seq on the myofibers were performed in biological replicates; ( $n=3$ MDX myofibers, $n=4$ WT

681 myofibers)

Figure 1 - Figure Supplement 1: Quality control of ATAC-Seq libraries

683 (A) Bioanalyzer profile of an ATAC-Seq library prepared from 5000 MuSCs. (B) Example bioanalyzer

684 profile of ATAC-Seq library prepared from a single myofiber. (C) Representative picture of a ready to

685 sequence MuSC ATAC-seq library after size selection, visualized on an agarose gel. (D) Fold enrichment

686 for the TSS of MyoD compared to negative control region of Chromosome 17 qE5 between MuSC

687 ATAC-Seq libraries and untagmented DNA as seen by qPCR ( $\mathrm{n}=5,3$ biological replicates and 2 technical

688 duplicates), two-tailed t-test, error bars $= \pm$ SD. (E) Representative picture of a ready to sequence ATAC-

689 seq library from a single myofiber after size selection, visualized on an agarose gel. (F) qPCR for the TSS

690 of $M y o D$ compared with a negative control region of Chromosome $17 \mathrm{qE} 5$ for the ATAC-Seq libraries

691 prepared from single myofibers ( $n=3$, biological triplicates), two-tailed t-test, error bars $= \pm$ SD.

692

693 Figure 2 - Figure Supplement 1. IGV snapshots of non-myogenic genes

(A) Platelet and Endothelial Cell Adhesion Molecule 1 (Pecam1) expressed in endothelial cells. (B)

696 Resistin (Retn) as a marker of adipocytes. (C) CD45 expressed in hematopoietic cells. (D) CD90 (Thyl)

697 expressed in fibroblasts. (E) Lymphocyte antigen 6a (Ly6a) expressed in fibro/adipogenic progenitors

698 (FAPs). (F) Adhesion G protein-coupled receptor E1 (ADGRE1) gene expressed in macrophages. (G) The

699 housekeeping gene RPS2 used as a positive control. (H) Housekeeping gene TATA-Box Binding protein

$700 \quad(T b p)$ used as a positive control.

701 *ATAC-Seq was performed in biological replicates; ( $n=3$ MuSCs, $n=3$ injured myofibers, $n=2$ uninjured 702 myofibers)

703

704 Figure 2 - Figure Supplement 2: Correlation analysis between biological replicates of each condition

706 (A-C) IGV snapshots of genes expressed in myofibers and MuSCs for all the replicates of each condition 707 that were pooled together for further analysis. DNase-Seq track added to demonstrate correlation of the 708 myofiber ATAC-Seq with previously performed DNase-Seq on skeletal muscle. (A) Housekeeping gene 709 Gapdh. (B) The muscle creatine kinase (Ckm). (C) Myogenic Factor 5 (Myf5). (D-J) Scatter plot showing 
Sahinyan et al. 2021 manuscript

the Pearson correlation between the replicates. (K) Venn diagram of the number of smfATAC-Seq peaks that are unique or overlapping with the peaks from ChIP-Seq of H3K27ac performed on EDL muscle. *ATAC-Seq was performed in biological replicates; $(n=3$ MuSCs, $n=3$ injured myofibers, $n=2$ uninjured myofibers)

** The ChIP-Seq data was retrieved from "Dynamic enhancers control skeletal muscle identity and reprogramming, PLoS Biology, Ramachandran, et al. 2019.” (43). This data is accessible through the GEO accession numbers GSM3515022 and GSM3515023.

\section{Figure 2 - Figure Supplement 3: IGV snapshots of Myogenic Regulatory Factors (MRFs)}

(A)Myogenic Factor 5 (Myf5). (B) MyoD. (C) Myogenin (Myog). (D) Myogenic factor 6 (Myf6).

*ATAC-Seq was performed in biological replicates $(n=3$ MuSCs, $n=3$ injured myofibers, $n=2$ uninjured myofibers)

\section{Figure 3 - Figure Supplement 1: Correlation analysis between uninjured and injured myofibers only}

(A)Heatmap clustering of Pearson correlation coefficients showing the correlation between the replicates of the injured and uninjured conditions in the regions defined by the union peakset (merged peaks of all replicates/samples). (B) Projection of the myofiber samples along first two principal components found by PCA showing the separate clustering of injured and uninjured myofibers.

*ATAC-Seq was performed in biological replicates; ( $n=3$ MuSCs, $n=3$ injured myofibers, $n=2$ uninjured myofibers)

Figure 3 - Figure Supplement 2. IGV snapshots of genes expressed in fast and slow muscle fiber types

(A) Troponin I2 (Tnni2) expressed in fast skeletal muscle fiber. (B) Troponin T3 (Tnnt3) expressed in fast skeletal muscle fiber. (C) Troponin T1 (Tnnt1) expressed in slow skeletal muscle fibers. (D) Myosin heavy chain $7(M y h 7)$ expressed in slow skeletal muscle fibers

*ATAC-Seq was performed in biological replicates; ( $n=3$ MuSCs, $n=3$ injured myofibers, $n=2$ uninjured myofibers)

Figure 3 - Figure Supplement 3: Unique peaks between different conditions indicate a distinct chromatin state for each cell type 
Sahinyan et al. 2021 manuscript

(A Peak score distribution (calculated by MACS2 peak calling algorithm) for each of the different conditions. Peak score $=-\log 10$ (FDR). (B) Heatmap showing the read count $+/-500 \mathrm{bp}$ of the center of

743 unique peaks to MuSCs compared to uninjured myofibers. (C) Heatmap showing the read count $+/-500$

744 bp of the center of unique peaks to injured myofibers compared to uninjured myofibers.

745 *ATAC-Seq was performed in biological replicates; $(n=3$ MuSCs, $n=3$ injured myofibers, $n=2$ uninjured

746 myofibers)

Figure 4 - Figure Supplement 1: Gene Ontology analysis of unique and common peaks between conditions

(A) Gene Ontology (GO Biological Process) analysis of genes associated with unique peaks present in the uninjured myofiber compared to MuSCs, based on the proximity of the peaks to the genes. (B) GO term analysis of genes associated with unique peaks in MuSCs compared to uninjured myofibers. (C) GO term GO term analysis of genes associated with peaks that are common between injured and uninjured myofibers. (E) GO term analysis of genes associated with unique peaks in injured myofibers compared to uninjured myofibers. (F) GO term analysis of genes associated with unique peaks in uninjured myofibers compared to injured myofibers.

*ATAC-Seq was performed in biological replicates; ( $n=3$ MuSCs, $n=3$ injured myofibers, $n=2$ uninjured myofibers)

Figure 4 - Figure Supplement 2: Top enriched motifs in the ATAC-Seq peaks of uninjured and injured myofibers

(A)Top 10 significantly enriched motifs in the peaks that are common between uninjured and injured myofibers overlapping the promoters $(+/-5 \mathrm{~kb})$. (B) Top 10 significantly enriched motifs in the peaks that are unique to injured myofibers overlapping the promoters $(+/-5 \mathrm{~kb})$. Figure 5 - Figure Supplement 1: Analysis of Notch and TGF $\beta$ signalling pathways reveal differential

(A)Heatmap showing genes involved in the Notch signalling pathway based on read counts of MuSCs,

773 uninjured fibers and injured fibers, $+/-1 \mathrm{~kb}$ of the TSS of each gene in the pathway. (B) IGV snapshot of

774 Notch homolog 1 (Notch1). (C) IGV snapshot of Protein jagged 2 (Jag2). (D) Heatmap showing genes

775 involved in the TGF $\beta$ signalling pathway based on read counts of MuSCs, uninjured fibers and injured 
Sahinyan et al. 2021 manuscript

fibers, +/- $1 \mathrm{~kb}$ of the TSS of each gene in the pathway. (E) IGV snapshot of Noggin (Nog). (F) IGV snapshot of Bone morphogenetic protein (Bmp4).

778 *ATAC-Seq was performed in biological replicates; $(n=3$ MuSCs, $n=3$ injured myofibers, $n=2$ uninjured

779 myofibers)

Figure 6 - Figure Supplement 1: Correlation analysis between biological replicates of mdx and WT myofiber ATAC-Seq samples

(A-I) Scatter plot showing the Pearson correlation between the replicates. (J,K) IGV snapshots of muscle creatine kinase $(\mathrm{Ckm})$ and housekeeping gene $\mathrm{Rps} 2$ for all the replicates of each condition that were pooled together for further analysis.

*ATAC-Seq on the myofibers were performed in biological replicates; ( $n=3$ mdx myofibers, $n=4$ WT myofibers)

Figure 6 - Figure Supplement 2: IGV snapshots of myogenic and non-myogenic genes for the quality control of mdx and WT smfATAC-Seq

(A-F) IGV snapshots of genes known to expressed in muscle fiber displaying accessibility on their respective TSS. (A) The muscle creatine kinase (Ckm). (B) Actin alpha 1 (Actal). (C) Part of the myosin heavy chain (Myh) gene cluster. (D) Housekeeping gene Gapdh. (E) Housekeeping gene Rps2. (F) POU Class 5 homeobox 1 (Pou5f1) as a negative control. (G-H) IGV snapshots of marker genes for fast and slow type myofibers. (G) Troponin I2 (Tnni2) expressed in fast skeletal muscle fiber. (H) Troponin T1 (Tnnt1) expressed in slow skeletal muscle fibers. (I-N) IGV snapshots of non-myogenic genes (I) Adhesion G protein-coupled receptor E1 (ADGRE1) gene expressed in macrophages. (J) Resistin (Retn) as a marker of adipocytes. (K) CD45 expressed in hematopoietic cells. (L) Lymphocyte antigen 6a ( $L y 6 a)$ expressed in fibro/adipogenic progenitors (FAPs). (M) Platelet and Endothelial Cell Adhesion Molecule 1 (Pecam1) expressed in endothelial cells. (N) CD90 (Thyl) expressed in fibroblasts.

*ATAC-Seq on the myofibers were performed in biological replicates; ( $n=3$ mdx myofibers, $n=4$ WT myofibers)

Figure 6 - Figure Supplement 3: Gene Ontology analysis of total mdx and WT peaks mdx myofiber, based on the proximity of the peaks to the genes. (B) GO term analysis of genes associated 
Sahinyan et al. 2021 manuscript

810 with all peaks present in the WT myofiber. (C) GO term analysis of genes associated with peaks that are

811 common between mdx and WT myofibers.

812 *ATAC-Seq on the myofibers were performed in biological replicates; ( $n=3$ mdx myofibers, $n=4$ WT

813 myofibers)

814

815 Figure 6 - Figure Supplement 4: Correlation analysis between injured, mdx and WT myofibers

816 (A)Heatmap clustering of Pearson correlation coefficients showing the correlation between the replicates

817 of the conditions in the regions defined by the union peakset (merged peaks of all replicates/samples). (B)

818 Projection of injured myofibers as well as the mdx and WT myofibers along the first two principal

819 components found by PCA.

820 *ATAC-Seq was performed in biological replicates; ( $n=3$ injured myofibers, $n=3$ mdx myofibers, $n=4$

821 WT myofibers)

822

823 Figure 6 - Figure Supplement 5: Top enriched motifs in the ATAC-Seq peaks of mdx and WT 824 myofibers

825

(A)Top 10 significantly enriched motifs in the peaks that are common between mdx and WT myofibers overlapping the promoters $(+/-5 \mathrm{~kb})$. (B) Top 10 significantly enriched motifs in the peaks that are unique to mdx myofibers overlapping the promoters (+/- $5 \mathrm{~kb}$ ). (C) Top 10 significantly enriched motifs in the peaks that are unique to WT myofibers overlapping the promoters $(+/-5 \mathrm{~kb})$.

\section{Source Data:}

832 (A) Unlabeled agarose gel (1.25\%) of MuSC ATAC-Seq sequence ready libraries. (B) Unlabeled 833 agarose gel (1.25\%) of uninjured myofiber ATAC-Seq sequence ready library. (C) Labeled 834 agarose gel (1.25\%) image of MuSC and uninjured myofiber ATAC-Seq sequence ready 835 libraries. (D) Raw file of bioanalyzer results from single myofiber sequence ready ATAC-Seq 836 libraries. 
Sahinyan et al. 2021 manuscript

845 MATERIALS AND METHODS

\begin{tabular}{|c|c|c|c|c|}
\hline \multicolumn{5}{|c|}{ Key Resources Table } \\
\hline $\begin{array}{l}\text { Reagent type } \\
\text { (species) or } \\
\text { resource }\end{array}$ & Designation & $\begin{array}{l}\text { Source or } \\
\text { reference }\end{array}$ & Identifiers & $\begin{array}{l}\text { Additional } \\
\text { information }\end{array}$ \\
\hline $\begin{array}{l}\text { Genetic reagent (M. } \\
\text { Musculus) }\end{array}$ & C57BL/6J & $\begin{array}{l}\text { The Jackson } \\
\text { Laboratory }\end{array}$ & Stock \#: 000664 & \\
\hline $\begin{array}{l}\text { Genetic reagent (M. } \\
\text { Musculus) }\end{array}$ & C57BL/10ScSnJ & $\begin{array}{l}\text { The Jackson } \\
\text { Laboratory }\end{array}$ & Stock \#: 000476 & \\
\hline $\begin{array}{l}\text { Genetic reagent ( } M \text {. } \\
\text { Musculus) }\end{array}$ & $\begin{array}{l}\text { C57BL/10ScSn- } \\
D m d^{\mathrm{mdx} / J}\end{array}$ & $\begin{array}{l}\text { The Jackson } \\
\text { Laboratory }\end{array}$ & Stock \#: 001801 & \\
\hline $\begin{array}{l}\text { Genetic reagent ( } M . \\
\text { Musculus) }\end{array}$ & $\begin{array}{l}\text { Tg(Pax7- } \\
\text { EGFE)\#Tagb } \\
\text { (Pax7-nGFP) }\end{array}$ & $\begin{array}{l}\text { Sambasivan, R. } \\
\text { et al. Distinct } \\
\text { Regulatory } \\
\text { Cascades } \\
\text { Govern } \\
\text { Extraocular and } \\
\text { Pharyngeal Arch } \\
\text { Muscle } \\
\text { Progenitor Cell } \\
\text { Fates. } \\
\text { Developmental } \\
\text { Cell, (2009). } \\
\text { (82) }\end{array}$ & PMID: 19531352 & $\begin{array}{l}\text { Dr. Shahragim } \\
\text { Tajbakhsh } \\
\text { (Institut Pasteur) }\end{array}$ \\
\hline $\begin{array}{l}\text { Commercial kit or } \\
\text { assay }\end{array}$ & Tn5 transposase & Illumina & Cat \#: 20034197 & \\
\hline $\begin{array}{l}\text { Commercial kit or } \\
\text { assay }\end{array}$ & $\begin{array}{l}\text { Nextera XT } \\
\text { adaptors }\end{array}$ & Illumina & Cat \#: FC-131-1001 & \\
\hline
\end{tabular}


Sahinyan et al. 2021 manuscript

\begin{tabular}{|c|c|c|c|c|}
\hline $\begin{array}{l}\text { Commercial kit or } \\
\text { assay }\end{array}$ & $\begin{array}{l}\text { QIAquick PCR } \\
\text { purification kit }\end{array}$ & Qiagen & Cat \#: 28104 & \\
\hline $\begin{array}{l}\text { Chemical } \\
\text { compound, drug }\end{array}$ & Triton $X-100$ & Sigma-Aldrich & Cat \#: T9284 & \\
\hline $\begin{array}{l}\text { Chemical } \\
\text { compound, drug }\end{array}$ & Tween-20 & Sigma-Aldrich & Cat \#: P1379-1L & \\
\hline $\begin{array}{l}\text { Chemical } \\
\text { compound, drug }\end{array}$ & Digitonin & Promega & Cat \#: G9441 & \\
\hline $\begin{array}{l}\text { Chemical } \\
\text { compound, drug }\end{array}$ & Collagenase D & Roche & Cat \#: 11088882001 & $2.4 \mathrm{U} / \mathrm{mL}$ \\
\hline $\begin{array}{l}\text { Chemical } \\
\text { compound, drug }\end{array}$ & Collagenase & Sigma-Aldrich & Cat \#: C0130 & $1000 \mathrm{U} / \mathrm{mL}$ \\
\hline $\begin{array}{l}\text { Chemical } \\
\text { compound, drug }\end{array}$ & Dispase II & Roche & Cat \#: 39307800 & $12 \mathrm{U} / \mathrm{mL}$ \\
\hline $\begin{array}{l}\text { Chemical } \\
\text { compound, drug }\end{array}$ & Cardiotoxin & Sigma Aldrich & Cat \#: 11061-96-4 & \\
\hline $\begin{array}{l}\text { Sequenced-based } \\
\text { reagent }\end{array}$ & MyoD_L & This paper & PCR primers & $\begin{array}{l}\text { TGCTCCTTTG } \\
\text { AGACAGCAG } \\
\text { A }\end{array}$ \\
\hline $\begin{array}{l}\text { Sequenced-based } \\
\text { reagent }\end{array}$ & MyoD_R & This paper & PCR primers & $\begin{array}{l}\text { AGTAGGGAA } \\
\text { GTGTGCGTGC } \\
\mathrm{T}\end{array}$ \\
\hline other & $\begin{array}{l}\text { Q5 High Fidelity } \\
\text { DNA polymerase }\end{array}$ & $\begin{array}{l}\text { New England } \\
\text { Biolabs }\end{array}$ & Cat \#: M0491S & $\begin{array}{l}\text { For } \\
\text { amplification } \\
\text { of DNA post } \\
\text { Tn5 } \\
\text { tagmentation } \\
\text { (see Library } \\
\text { Preparation) }\end{array}$ \\
\hline $\begin{array}{l}\text { Chemical } \\
\text { compound }\end{array}$ & DAPI stain & Invitrogen & Cat \#: D3671 & $(5 \mathrm{mg} / \mathrm{mL})$ \\
\hline other & Ampure XP beads & Beckman & Cat \#: A63880 & $\begin{array}{l}\text { For library size } \\
\text { selection at a } \\
\text { concentration of } \\
0.85 x \text { (see } \\
\text { Library }\end{array}$ \\
\hline
\end{tabular}


Sahinyan et al. 2021 manuscript

\begin{tabular}{|l|l|l|l|l|}
\hline & & & & Preparation) \\
\hline $\begin{array}{l}\text { Chemical } \\
\text { compound }\end{array}$ & Hoechst & $\begin{array}{l}\text { Molecular } \\
\text { Probes }\end{array}$ & Cat \#: H1399 & $(5 \mathrm{mg} / \mathrm{mL})$ \\
\hline
\end{tabular}

ATAC-Seq on a Single Myofiber

Isolation of Extensor Digitorum Longus (EDL) from cardiotoxin-induced injured muscle

The Extensor Digitorum Longus (EDL) muscle was injured by intramuscular injection of $50 \mu \mathrm{L}$ of $5 \mu \mathrm{M}$ cardiotoxin (CTX) (Sigma, 11061-96-4). Mice were treated with carprofen 20 minutes prior to CTX injection and were injected with CTX under anesthesia by isoflurane. Mice were sacrificed seven days post injury and the EDL was collected from the hind limb of each mouse with the contra lateral EDL being used for the isolation of uninjured myofibers.

\section{Dissection of EDL muscle}

The EDL muscle was dissected as previously described (44). Briefly, the skin of the hindlimb was removed and the tibialis anterior (TA) muscle was excised with a pair of dissection scissors. The tendons of the EDL were exposed and the EDL was cut from tendon to tendon with scissors.

\section{Isolation of a single EDL myofiber}

861 Individual myofibers were isolated from the EDL muscle as previously described (44). Briefly, the intact

862 EDL muscle was placed in a $1.5 \mathrm{~mL}$ eppendorf tube with $800 \mu \mathrm{L}$ of myofiber digestion buffer containing $8631000 \mathrm{U} / \mathrm{mL}$ of collagenase from Clostridium histolyticum (Sigma, C0130) in un-supplemented DMEM

864 (Gibco, 11995-065) for 1 hour. Trypsin was added to the myofiber digestion buffer at a final

865 concentration of $0.25 \%$ to remove the myofiber associated muscle stem cells. The EDL myofibers were

866 then transferred into $2 \mathrm{~mL}$ of $1 \mathrm{X}$ PBS (Wisent, 311-425-CL) in a 6 well-plate that had previously been

867 coated with DMEM supplemented with 10\% horse serum (HS) (Wisent, 065250). The EDL was then

868 gently pipetted up and down with a large-bore glass pipette to disassociate the myofibers.

\section{Selection of injured and uninjured myofibers}

871 Live myofibers in the 6-well plate were stained with $2 \mu \mathrm{L}$ of $5 \mathrm{mg} / \mathrm{mL}$ of Hoechst (Molecular Probes, $872 \mathrm{H} 1399)$ in $2 \mathrm{~mL}$ of $1 \mathrm{X}$ PBS for 5 minutes in a $37^{\circ} \mathrm{C}$ with $5 \% \mathrm{CO}_{2}$ incubator. The myofibers were then 
Sahinyan et al. 2021 manuscript

873 visualized under a microscope and selected based on the myonuclei location, where myofibers with a

874 pattern of centrally located nuclei were determined to be regenerating and picked for the injury condition.

875 Individual myofibers were then transferred to $0.2 \mathrm{~mL}$ microtubes using a small-bore glass pipette coated

876 with HS.

877

878 Lysis and permeabilization of the myofiber

879 Residual media was removed with a pipette under a microscope. Individual myofibers in $0.2 \mathrm{~mL}$

880 microtubes were put in $10 \mu \mathrm{L}$ of $\mathrm{ddH}_{2} \mathrm{O}$ for 5 minutes on ice. The $\mathrm{ddH}_{2} \mathrm{O}$ was removed with a pipette

881 under a microscope, ensuring that the myofiber remained in the tube. The myofiber was then

882 permeabilized with $20 \mu \mathrm{L}$ of $0.5 \%$ Triton X-100 (Sigma, T9284) in PBS for 15 minutes at room

883 temperature (RT). The permeabilization buffer was removed with a pipette under a microscope and the

884 myofiber was washed twice with $200 \mu \mathrm{L}$ of $1 \mathrm{X}$ PBS.

885

\section{Tagmentation of the myofiber by Tn5 transposase}

Transposition and ATAC-seq library preparation for a single myofiber was adapted from previously described OMNI ATAC-Seq protocol (30). The permeabilized myonuclei were tagmented with tagmentation mixture optimized for use on a myofiber (20 $\mu \mathrm{L}$ Tagment DNA Buffer (TD Buffer) (Illumina, 20034197), 13.3 $\mu$ L PBS, 0.2\% Tween-20 (Sigma, P1379-1L), 0.02\% Digitonin (Promega,

$8926 \mu \mathrm{L}$ of the tagmentation mixture at $37^{\circ} \mathrm{C}$ for 56 minutes with periodic shaking of the tubes every $5-7$

893 minutes. Following the transposition with Tn5, DNA was purified using a QIAquick PCR Purification Kit 894 (Qiagen, 28104) according to the manufacturer's guidelines.

\section{Library preparation}

897 The purified DNA was PCR amplified for 15 cycles using Q5 High Fidelity DNA polymerase (New

898 England Biolabs, M0491S) with the incorporation of Illumina Nextera XT adaptors (Illumina, FC-131-

899 1001). The libraries were then size selected with AmpureXP Beads (Beckman, Cat\# A63880) at a 1: 0.85

900 ratio $(\mathrm{v} / \mathrm{v})$. The size selected libraries were verified for quality control by bioanalyzer as well as

901 verification of the library size via visualization on an agarose gel stained with GelGreen dye (Biotium,

902 41005). Libraries were then sequenced on NovaSeq6000 Sprime Paired End (PE) 150 bp.

\section{ATAC-Seq on MuSCs}

904 Isolation of MuSCs by fluorescence-activated cell sorting (FACS) for ATAC-Seq 
Sahinyan et al. 2021 manuscript

905

906

907

908

909

910

911

912

913

914

915

916

917

918

919

920

921

922

923

924

925

926

927

928

929

930

931

932

933

934

935

936

937

MuSCs were isolated by Fluorescence Activated Cell Sorting (FACS) as previously described (83).

Briefly, hindlimb muscles from Pax $7 / \mathrm{GFP}^{+}$mice were dissected and chopped. The minced muscles were then transferred into a $15 \mathrm{~mL}$ Falcon tube and digested in un-supplemented F10 media (Gibco, 11550043) with 2.4 U/mL Collagenase D (Roche, 11088882001), 12 U/mL Dispase II (Roche, 39307800) and $0.5 \mathrm{mM} \mathrm{CaCl}_{2}$. Digestion was performed on a shaker in an incubator at $37{ }^{\circ} \mathrm{C}$ with $5 \% \mathrm{CO}_{2}$ for 30 minutes. Following the first digestion, digested muscles were centrifuged at $600 \mathrm{~g}$ for 20 seconds and the supernatant was transferred to a $50 \mathrm{~mL}$ Falcon tube with $9 \mathrm{~mL}$ FBS (Wisent, 080450) and was kept on ice. The remaining pellet was triturated and was digested for another 15 minutes with additional digestion buffer added. After the final digestion, the digested muscle mixture was transferred to the $50 \mathrm{ml}$ Falcon tube containing the previously digested mixture. The digested muscle mixture was then filtered through a $40 \mu \mathrm{m}$ cell strainer (Falcon, C352340) and was centrifuged at $600 \mathrm{~g}$ for 18 minutes at $4^{\circ} \mathrm{C}$. The pelleted cells were then resuspended in $800 \mu \mathrm{L}$ FACS buffer that is composed of $2 \%$ FBS/ PBS (v:v), $0.5 \mathrm{mM}$ EDTA (Invitrogen, AM9261) and with $0.5 \mu \mathrm{L}$ DAPI (5mg/mL) (Invitrogen, D3671). Resuspended cells were then filtered through $40 \mu \mathrm{m}$ cell strainer and were transferred into polypropylene round-bottom FACS compatible tubes (Falcon, 352063). MuSCs were sorted with a FACSAria Fusion cytometer (BD Biosciences) based on negative selection for DAPI and positive selection for GFP.

\section{Lysis and transposition of MuSCs}

ATAC-Seq on MuSCs was performed based on the previously established OMNI-ATAC-Seq protocol (30). Briefly, five thousand MuSCs were sorted by FACS into $30 \mu \mathrm{L}$ of the ATAC lysis buffer containing 10 mM Tris-HCl (pH 7.5), 10 mM NaCl (Bioshop, 7647-14-5), 3 mM MgCl2 (Sigma, 7786-30-3), 0.1\% Tween-20 (Sigma, P1379-1L), 0.1\% NP-40 (Sigma, 74385), and 0.01\% Digitonin (Promega, G9441) in a $0.2 \mathrm{~mL}$ microtube. Cells were incubated in the lysis buffer for 5 minutes on ice and then 3 minutes at room temperature (RT). Cells were then washed with $100 \mu \mathrm{L}$ of wash buffer composed of $10 \mathrm{mM}$ Tris$\mathrm{HCl}(\mathrm{pH} 7.5), 10 \mathrm{Mm} \mathrm{NaCl}, 3 \mathrm{mM} \mathrm{MgCl} 2$ and $0.1 \%$ Tween-20, and were centrifuged at $800 \mathrm{~g}$ for 10 minutes. The pellet was resuspended in $10 \mu \mathrm{L}$ of transposition mixture $(5 \mu \mathrm{L}$ TD buffer, $3.2 \mu \mathrm{L}$ PBS, 0.89 $\mu \mathrm{L}$ Tn5 (Illumina, 20034197), 0.1\% Tween-20, 0.01\% Digitonin and $0.75 \mu \mathrm{L}$ nuclease free water).

Transposition was performed for 20 minutes at $37^{\circ} \mathrm{C}$ while shaking the tubes every 5-7 minutes. The DNA was then purified using a QIAquick PCR Purification Kit according to the manufacturer's guidelines.

\section{Library preparation for MuSCs ATAC-Seq}

The eluted tagmented DNA was PCR amplified for 12 cycles with the incorporation of Illumina Nextera XT adapters using Q5 High Fidelity DNA polymerase. The libraries were then size selected with 
Sahinyan et al. 2021 manuscript

938 AmpureXP Beads at a 1: 0.85 ratio (v/v). The libraries were then verified by bioanalyzer and agarose gel

939 visualization. Finally, the samples were sequenced on NovaSeq6000 Sprime Paired End (PE) 150 bp.

$940 \quad$ ATAC-Seq data processing

941 The sequencing data was processed using the GenPipes pipeline v.3.1.5 (84). The raw reads were

942 trimmed using Trimmomatic v.0.36 (85) and aligned to the mm10 genome assembly using the Burrows-

943 Wheeler Aligner v.0.7.12 (86). Reads were filtered to keep only high quality alignments (MAPQ score >

944 20) and duplicates were removed using SAMtools v.1.3.1 (87). Peak calling was performed with MACS2

945 v.2.1.1 (88) using piling up of paired-end fragment mode (--format BAMPE). The peak files (bed) were

946 filtered by removing the ENCODE black listed regions

947 (https://www.encodeproject.org/files/ENCFF547MET) using BEDTools v2.29.1 (89). Mitochondrial

948 reads were also removed before the analysis.

\section{Correlation analysis between the biological replicates and clustering}

950 In order to perform a quantitative comparison of the read counts within accessible regions, the

951 overlapping peaks of all replicates were merged using BEDTools v2.29.1 (89). This set of merged peaks

952 and the BAM alignment files were used as input for the featureCounts function of Rsubread v.2.2.6 (90)

953 to generate a raw-count matrix. The raw counts were normalized by rlog transformation using DESeq2

954 (91) with respect to library size. Pearson correlation coefficients were calculated based on the normalized

955 counts for each pairwise comparison. Principal component analysis (PCA) and hierarchical clustering

956 were also performed to evaluate the similarity between the replicates.

957 Peak annotation analysis

958 For each condition, the BAM alignment files of the replicates were merged and peak calling was

959 performed with MACS2 v.2.1.1 (88). Peak sets for each condition were annotated using the ChIPseaker

960 v.1.24.0 (92) annotatePeak function, and the UCSC Genome Browser knownGene (mm10) table.

961

962 Obtaining coverage tracks

963 The BAM alignment files were converted to bigWig format and normalized by scaling factor (--

964 scaleFactor) with the deepTools v.2.5.0.1 (93) bamCoverage function.

965

966 Enrichment of genomic signal around TSS 
Sahinyan et al. 2021 manuscript

967 The bigWig files and the TSS coordinates obtained from the UCSC Genome Browser knownGene

968 (mm10) table were used as input for the computeMatrix function of deepTools v.2.5.0.1 (93). This matrix

969 was used for plotHeatmap function to generate the heatmap.

970

971 Identification of overlapping/unique accessible regions

972 For each comparison between the conditions, overlapping and unique accessible regions were identified

973 with DiffBind v.2.16.2(94) based on the measure of confidence in the peak call by MACS2 v.2.1.1 (88).

974

\section{Analysis of differentially accessible regions}

977 The identification of differentially accessible regions (DARs) between the conditions was done using 978 DiffBind v.2.16.2 (94) and edgeR v.3.30.1 (95). Log fold changes were calculated, and their associated p979 values were corrected for multiple hypothesis testing via the Benjamini-Hochberg procedure to obtain 980 adjusted p-values. The DARs were annotated by their nearest gene using the annotatePeaks.pl function of 981 Homer v.4.11(96).

982

\section{Gene set enrichment analysis}

Genes nearby the DARs were ranked based on the log-fold change calculated with edgeR v.3.30.1 (95).

This ranked list of genes was used as input to perform gene set enrichment analysis with the

fgseaMultilevel function of the R package fgsea v.1.14.0 (97). The FGSEA-multilevel method is based on an adaptive multi-level split Monte Carlo scheme, which allows the estimation of very low p-values. The Hallmark gene sets collection from the Molecular Signatures Database (MSigDB) (97) was used as a reference to identify the biological processes that were significantly enriched.

995 was done using the findMotifsGenome.pl function from HOMER v.4.9.1 (96). The -size parameter was set 996 to given to use the exact peak region as target sequence. Following the screening of HOMER's reliable 997 motifs library against the target sequences, the motifs enriched with a p-value less than 0.05 are returned.

\section{Animal care}

1000 All procedures that were performed on animals were approved by the McGill University Animal Care 1001 Committee (UACC), protocol \#7512.

\section{Data availability}


Sahinyan et al. 2021 manuscript

1003 The data discussed in this study have been deposited in NCBI's Gene Expression Omnibus and are

1004 accessible through GEO Series accession numbers GSE173676 and GSE171534, tokens

1005 edunwycudrgbfmj and insxmyaydpezjcz respectively.

1006

1007

1008 Impact Statement

1009 Single myofiber ATAC-Seq provides assessment of chromatin accessibility of a single myofiber without

1010 the confounding effects of other cell types present in skeletal muscle.

1011

\section{Acknowledgments}

1013 We thank Christian Young at the Lady Davis Institute for Medical Research-Jewish General Hospital-

1014 core facility for his help with fluorescence-activated cell sorting (FACS) of muscle stem cells. We thank

1015 Dr. Michael Witcher at McGill University Department of Oncology for his critical comments and careful 1016 review of an early draft of this manuscript.

1017

1018 Funding

1019 This work was supported by a discovery grant from Natural Sciences and Engineering Research Council

1020 (NSERC) to VDS.

1021

1022 Declaration of Interests

1023 The authors declare no competing interests.

1024

1025

1026

1027

1028

1029

1030

1031

1032

1033

1034

1035

1036

1037

1038

1039

\section{References}

1. Buckingham, M., Bajard, L., Chang, T., Daubas, P., Hadchouel, J., Meilhac, S., Montarras, D., Rocancourt, D., and Relaix, F. (2003) The formation of skeletal muscle: from somite to limb. J Anat 202, 59-68

2. Huxley, H., and Hanson, J. (1954) Changes in the cross-striations of muscle during contraction and stretch and their structural interpretation. Nature 173, 973-976

3. Zierath, J. R., and Hawley, J. A. (2004) Skeletal muscle fiber type: influence on contractile and metabolic properties. PLoS Biol 2, e348 
Sahinyan et al. 2021 manuscript

10404 4. Bohe, J., Low, J. F., Wolfe, R. R., and Rennie, M. J. (2001) Latency and duration of

5. Mittendorfer, B., Andersen, J. L., Plomgaard, P., Saltin, B., Babraj, J. A., Smith, K., and

10. Deschenes, M. R. (2004) Effects of aging on muscle fibre type and size. Sports Med 34, 1059

1060

1061

1062

1063

1064

1065

1066

1067

1068

1069

1070

1071

1072

1073

1074

1075

1076

1077

1078

1079

1080

1081 Rennie, M. J. (2005) Protein synthesis rates in human muscles: neither anatomical location nor fibre-type composition are major determinants. J Physiol 563, 203-211

6. Quiat, D., Voelker, K. A., Pei, J., Grishin, N. V., Grange, R. W., Bassel-Duby, R., and Olson, E. N. (2011) Concerted regulation of myofiber-specific gene expression and muscle performance by the transcriptional repressor Sox6. Proc Natl Acad Sci U S A 108, 1019610201

7. Weskamp, K., Olwin, B. B., and Parker, R. (2021) Post-Transcriptional Regulation in Skeletal Muscle Development, Repair, and Disease. Trends Mol Med 27, 469-481

8. Wilson, J. M., Loenneke, J. P., Jo, E., Wilson, G. J., Zourdos, M. C., and Kim, J. S. (2012) The effects of endurance, strength, and power training on muscle fiber type shifting. $J$ Strength Cond Res 26, 1724-1729

9. Dons, B., Bollerup, K., Bonde-Petersen, F., and Hancke, S. (1979) The effect of weightlifting exercise related to muscle fiber composition and muscle cross-sectional area in humans. Eur J Appl Physiol Occup Physiol 40, 95-106 809-824

11. Thompson, L. V. (2002) Skeletal muscle adaptations with age, inactivity, and therapeutic exercise. J Orthop Sports Phys Ther 32, 44-57

12. Nilwik, R., Snijders, T., Leenders, M., Groen, B. B., van Kranenburg, J., Verdijk, L. B., and van Loon, L. J. (2013) The decline in skeletal muscle mass with aging is mainly attributed to a reduction in type II muscle fiber size. Exp Gerontol 48, 492-498

13. Roberts, B. M., Frye, G. S., Ahn, B., Ferreira, L. F., and Judge, A. R. (2013) Cancer cachexia decreases specific force and accelerates fatigue in limb muscle. Biochem Biophys Res Commun 435, 488-492

14. Lazure, F., Blackburn, D. M., Corchado, A. H., Sahinyan, K., Karam, N., Sharanek, A., Nguyen, D., Lepper, C., Najafabadi, H. S., Perkins, T. J., Jahani-Asl, A., and Soleimani, V. D. (2020) Myf6/MRF4 is a myogenic niche regulator required for the maintenance of the muscle stem cell pool. EMBO Rep 21, e49499

15. Snow, M. H. (1977) Myogenic cell formation in regenerating rat skeletal muscle injured by mincing. II. An autoradiographic study. Anat Rec 188, 201-217

16. Shadrach, J. L., and Wagers, A. J. (2011) Stem cells for skeletal muscle repair. Philos Trans $R$ Soc Lond B Biol Sci 366, 2297-2306

17. Dumont, N. A., Bentzinger, C. F., Sincennes, M. C., and Rudnicki, M. A. (2015) Satellite Cells and Skeletal Muscle Regeneration. Compr Physiol 5, 1027-1059

18. Giordani, L., He, G. J., Negroni, E., Sakai, H., Law, J. Y. C., Siu, M. M., Wan, R., Corneau, A., Tajbakhsh, S., Cheung, T. H., and Le Grand, F. (2019) High-Dimensional Single-Cell Cartography Reveals Novel Skeletal Muscle-Resident Cell Populations. Mol Cell 74, 609621 e606 
Sahinyan et al. 2021 manuscript

1082

1083

1084

1085

1086

1087

1088

1089

1090

1091

1092

1093

1094

1095

1096

1097

1098

1099

1100

1101

1102

1103

1104

1105

1106

1107

1108

1109

1110

1111

1112

1113

1114

1115

1116

1117

1118

1119

1120

1121

1122

1123

1124

1125

19. Petrany, M. J., Swoboda, C. O., Sun, C., Chetal, K., Chen, X., Weirauch, M. T., Salomonis, N., and Millay, D. P. (2020) Single-nucleus RNA-seq identifies transcriptional heterogeneity in multinucleated skeletal myofibers. Nat Commun 11, 6374

20. Dos Santos, M., Backer, S., Saintpierre, B., Izac, B., Andrieu, M., Letourneur, F., Relaix, F., Sotiropoulos, A., and Maire, P. (2020) Single-nucleus RNA-seq and FISH identify coordinated transcriptional activity in mammalian myofibers. Nat Commun 11, 5102

21. Kim, M., Franke, V., Brandt, B., Lowenstein, E. D., Schowel, V., Spuler, S., Akalin, A., and Birchmeier, C. (2020) Single-nucleus transcriptomics reveals functional compartmentalization in syncytial skeletal muscle cells. Nat Commun 11, 6375

22. Zhu, F., Farnung, L., Kaasinen, E., Sahu, B., Yin, Y., Wei, B., Dodonova, S. O., Nitta, K. R., Morgunova, E., Taipale, M., Cramer, P., and Taipale, J. (2018) The interaction landscape between transcription factors and the nucleosome. Nature 562, 76-81

23. Thurman, R. E., Rynes, E., Humbert, R., Vierstra, J., Maurano, M. T., Haugen, E., Sheffield, N. C., Stergachis, A. B., Wang, H., Vernot, B., Garg, K., John, S., Sandstrom, R., Bates, D., Boatman, L., Canfield, T. K., Diegel, M., Dunn, D., Ebersol, A. K., Frum, T., Giste, E., Johnson, A. K., Johnson, E. M., Kutyavin, T., Lajoie, B., Lee, B. K., Lee, K., London, D., Lotakis, D., Neph, S., Neri, F., Nguyen, E. D., Qu, H., Reynolds, A. P., Roach, V., Safi, A., Sanchez, M. E., Sanyal, A., Shafer, A., Simon, J. M., Song, L., Vong, S., Weaver, M., Yan, Y., Zhang, Z., Zhang, Z., Lenhard, B., Tewari, M., Dorschner, M. O., Hansen, R. S., Navas, P. A., Stamatoyannopoulos, G., Iyer, V. R., Lieb, J. D., Sunyaev, S. R., Akey, J. M., Sabo, P. J., Kaul, R., Furey, T. S., Dekker, J., Crawford, G. E., and Stamatoyannopoulos, J. A. (2012) The accessible chromatin landscape of the human genome. Nature 489, 75-82

24. Liu, L., Leng, L., Liu, C., Lu, C., Yuan, Y., Wu, L., Gong, F., Zhang, S., Wei, X., Wang, M., Zhao, L., Hu, L., Wang, J., Yang, H., Zhu, S., Chen, F., Lu, G., Shang, Z., and Lin, G. (2019) An integrated chromatin accessibility and transcriptome landscape of human preimplantation embryos. Nat Commun 10, 364

25. Trevino, A. E., Sinnott-Armstrong, N., Andersen, J., Yoon, S. J., Huber, N., Pritchard, J. K., Chang, H. Y., Greenleaf, W. J., and Pasca, S. P. (2020) Chromatin accessibility dynamics in a model of human forebrain development. Science 367

26. Corces, M. R., Granja, J. M., Shams, S., Louie, B. H., Seoane, J. A., Zhou, W., Silva, T. C., Groeneveld, C., Wong, C. K., Cho, S. W., Satpathy, A. T., Mumbach, M. R., Hoadley, K. A., Robertson, A. G., Sheffield, N. C., Felau, I., Castro, M. A. A., Berman, B. P., Staudt, L. M., Zenklusen, J. C., Laird, P. W., Curtis, C., Cancer Genome Atlas Analysis, N., Greenleaf, W. J., and Chang, H. Y. (2018) The chromatin accessibility landscape of primary human cancers. Science 362

27. Rendeiro, A. F., Schmidl, C., Strefford, J. C., Walewska, R., Davis, Z., Farlik, M., Oscier, D., and Bock, C. (2016) Chromatin accessibility maps of chronic lymphocytic leukaemia identify subtype-specific epigenome signatures and transcription regulatory networks. Nat Commun 7, 11938

28. Wang, Y., Zhang, X., Song, Q., Hou, Y., Liu, J., Sun, Y., and Wang, P. (2020) Characterization of the chromatin accessibility in an Alzheimer's disease (AD) mouse model. Alzheimers Res Ther 12, 29

29. Bastle, R. M., and Maze, I. (2019) Chromatin Regulation in Complex Brain Disorders. Curr Opin Behav Sci 25, 57-65 
Sahinyan et al. 2021 manuscript

1126

1127

1128

1129

1130

1131

1132

1133

1134

1135

1136

1137

1138

1139

1140

1141

1142

1143

1144

1145

1146

1147

1148

1149

1150

1151

1152

1153

1154

1155

1156

1157

1158

1159

1160

1161

1162

1163

1164

1165

1166

1167

1168

30. Corces, M. R., Trevino, A. E., Hamilton, E. G., Greenside, P. G., Sinnott-Armstrong, N. A., Vesuna, S., Satpathy, A. T., Rubin, A. J., Montine, K. S., Wu, B., Kathiria, A., Cho, S. W., Mumbach, M. R., Carter, A. C., Kasowski, M., Orloff, L. A., Risca, V. I., Kundaje, A., Khavari, P. A., Montine, T. J., Greenleaf, W. J., and Chang, H. Y. (2017) An improved ATAC-seq protocol reduces background and enables interrogation of frozen tissues. Nat Methods 14, 959-962

31. Buenrostro, J. D., Wu, B., Chang, H. Y., and Greenleaf, W. J. (2015) ATAC-seq: A Method for Assaying Chromatin Accessibility Genome-Wide. Curr Protoc Mol Biol 109, 212921 212929

32. Liu, C., Wang, M., Wei, X., Wu, L., Xu, J., Dai, X., Xia, J., Cheng, M., Yuan, Y., Zhang, P., Li, J., Feng, T., Chen, A., Zhang, W., Chen, F., Shang, Z., Zhang, X., Peters, B. A., and Liu, L. (2019) An ATAC-seq atlas of chromatin accessibility in mouse tissues. Sci Data 6, 65

33. Yan, F., Powell, D. R., Curtis, D. J., and Wong, N. C. (2020) From reads to insight: a hitchhiker's guide to ATAC-seq data analysis. Genome Biol 21, 22

34. Rocks, D., Jaric, I., Tesfa, L., Greally, J. M., Suzuki, M., and Kundakovic, M. (2021) Cell type-specific chromatin accessibility analysis in the mouse and human brain.

Epigenetics, 1-18

35. Janssen, I., Heymsfield, S. B., Wang, Z. M., and Ross, R. (2000) Skeletal muscle mass and distribution in 468 men and women aged 18-88 yr. J Appl Physiol (1985) 89, 81-88

36. Ortenblad, N., Nielsen, J., Boushel, R., Soderlund, K., Saltin, B., and Holmberg, H. C. (2018) The Muscle Fiber Profiles, Mitochondrial Content, and Enzyme Activities of the Exceptionally Well-Trained Arm and Leg Muscles of Elite Cross-Country Skiers. Front Physiol 9, 1031

37. Mishra, P., Varuzhanyan, G., Pham, A. H., and Chan, D. C. (2015) Mitochondrial Dynamics is a Distinguishing Feature of Skeletal Muscle Fiber Types and Regulates Organellar Compartmentalization. Cell Metab 22, 1033-1044

38. Neal, A., Boldrin, L., and Morgan, J. E. (2012) The satellite cell in male and female, developing and adult mouse muscle: distinct stem cells for growth and regeneration. PLoS One 7, e37950

39. Cramer, A. A. W., Prasad, V., Eftestol, E., Song, T., Hansson, K. A., Dugdale, H. F., Sadayappan, S., Ochala, J., Gundersen, K., and Millay, D. P. (2020) Nuclear numbers in syncytial muscle fibers promote size but limit the development of larger myonuclear domains. Nat Commun 11, 6287

40. McGreevy, J. W., Hakim, C. H., Mclntosh, M. A., and Duan, D. (2015) Animal models of Duchenne muscular dystrophy: from basic mechanisms to gene therapy. Dis Model Mech 8, 195-213

41. De Micheli, A. J., Laurilliard, E. J., Heinke, C. L., Ravichandran, H., Fraczek, P., SoueidBaumgarten, S., De Vlaminck, I., Elemento, O., and Cosgrove, B. D. (2020) Single-Cell Analysis of the Muscle Stem Cell Hierarchy Identifies Heterotypic Communication Signals Involved in Skeletal Muscle Regeneration. Cell Rep 30, 3583-3595 e3585

42. De Micheli, A. J., Spector, J. A., Elemento, O., and Cosgrove, B. D. (2020) A reference single-cell transcriptomic atlas of human skeletal muscle tissue reveals bifurcated muscle stem cell populations. Skelet Muscle 10, 19 
Sahinyan et al. 2021 manuscript

43. Ramachandran, K., Senagolage, M. D., Sommars, M. A., Futtner, C. R., Omura, Y., Allred, A. L., and Barish, G. D. (2019) Dynamic enhancers control skeletal muscle identity and reprogramming. PLoS Biol 17, e3000467

44. Blackburn, D. M., Lazure, F., Corchado, A. H., Perkins, T. J., Najafabadi, H. S., and Soleimani, V. D. (2019) High-resolution genome-wide expression analysis of single myofibers using SMART-Seq. J Biol Chem 294, 20097-20108

45. Khan, S. S., Solomon, M. A., and McCoy, J. P., Jr. (2005) Detection of circulating endothelial cells and endothelial progenitor cells by flow cytometry. Cytometry B Clin Cytom 64, 1-8

46. Steppan, C. M., Bailey, S. T., Bhat, S., Brown, E. J., Banerjee, R. R., Wright, C. M., Patel, H. R., Ahima, R. S., and Lazar, M. A. (2001) The hormone resistin links obesity to diabetes. Nature 409, 307-312

47. McKinney-Freeman, S. L., Naveiras, O., Yates, F., Loewer, S., Philitas, M., Curran, M., Park, P. J., and Daley, G. Q. (2009) Surface antigen phenotypes of hematopoietic stem cells from embryos and murine embryonic stem cells. Blood 114, 268-278

48. Agorku, D. J., Langhammer, A., Heider, U., Wild, S., Bosio, A., and Hardt, O. (2019) CD49b, CD87, and CD95 Are Markers for Activated Cancer-Associated Fibroblasts Whereas CD39 Marks Quiescent Normal Fibroblasts in Murine Tumor Models. Front Oncol 9, 716

49. Waddell, L. A., Lefevre, L., Bush, S. J., Raper, A., Young, R., Lisowski, Z. M., McCulloch, M. E. B., Muriuki, C., Sauter, K. A., Clark, E. L., Irvine, K. M., Pridans, C., Hope, J. C., and Hume, D. A. (2018) ADGRE1 (EMR1, F4/80) Is a Rapidly-Evolving Gene Expressed in Mammalian Monocyte-Macrophages. Front Immunol 9, 2246

50. Joe, A. W., Yi, L., Natarajan, A., Le Grand, F., So, L., Wang, J., Rudnicki, M. A., and Rossi, F. M. (2010) Muscle injury activates resident fibro/adipogenic progenitors that facilitate myogenesis. Nat Cell Biol 12, 153-163

51. Folker, E. S., and Baylies, M. K. (2013) Nuclear positioning in muscle development and disease. Front Physiol 4, 363

52. Roman, W., and Gomes, E. R. (2018) Nuclear positioning in skeletal muscle. Semin Cell Dev Biol 82, 51-56

53. Tai, P. W., Fisher-Aylor, K. I., Himeda, C. L., Smith, C. L., Mackenzie, A. P., Helterline, D. L., Angello, J. C., Welikson, R. E., Wold, B. J., and Hauschka, S. D. (2011) Differentiation and fiber type-specific activity of a muscle creatine kinase intronic enhancer. Skelet Muscle 1, 25

54. Rudnicki, M. A., Schnegelsberg, P. N., Stead, R. H., Braun, T., Arnold, H. H., and Jaenisch, R. (1993) MyoD or Myf-5 is required for the formation of skeletal muscle. Cell 75, 13511359

55. Zhang, T., Cooper, S., and Brockdorff, N. (2015) The interplay of histone modifications writers that read. EMBO Rep 16, 1467-1481

56. Berger, S. L. (2007) The complex language of chromatin regulation during transcription. Nature 447, 407-412

57. Barrera, L. O., Li, Z., Smith, A. D., Arden, K. C., Cavenee, W. K., Zhang, M. Q., Green, R. D., and Ren, B. (2008) Genome-wide mapping and analysis of active promoters in mouse embryonic stem cells and adult organs. Genome Res 18, 46-59 
Sahinyan et al. 2021 manuscript

1213

1214

1215

1216

1217

1218

1219

1220

1221

1222

1223

1224

1225

1226

1227

1228

1229

1230

1231

1232

1233

1234

1235

1236

1237

1238

1239

1240

1241

1242

1243

1244

1245

1246

1247

1248

1249

1250

1251

1252

1253

1254

1255

1256

58. Nowak, K. J., Wattanasirichaigoon, D., Goebel, H. H., Wilce, M., Pelin, K., Donner, K., Jacob, R. L., Hubner, C., Oexle, K., Anderson, J. R., Verity, C. M., North, K. N., Iannaccone, S. T., Muller, C. R., Nurnberg, P., Muntoni, F., Sewry, C., Hughes, I., Sutphen, R., Lacson, A. G., Swoboda, K. J., Vigneron, J., Wallgren-Pettersson, C., Beggs, A. H., and Laing, N. G. (1999) Mutations in the skeletal muscle alpha-actin gene in patients with actin myopathy and nemaline myopathy. Nat Genet 23, 208-212

59. Stuart, C. A., Stone, W. L., Howell, M. E., Brannon, M. F., Hall, H. K., Gibson, A. L., and Stone, M. H. (2016) Myosin content of individual human muscle fibers isolated by laser capture microdissection. Am J Physiol Cell Physiol 310, C381-389

60. Seale, P., Sabourin, L. A., Girgis-Gabardo, A., Mansouri, A., Gruss, P., and Rudnicki, M. A. (2000) Pax7 is required for the specification of myogenic satellite cells. Cell 102, 777-786

61. Hernandez-Hernandez, J. M., Garcia-Gonzalez, E. G., Brun, C. E., and Rudnicki, M. A. (2017) The myogenic regulatory factors, determinants of muscle development, cell identity and regeneration. Semin Cell Dev Biol 72, 10-18

62. Montarras, D., Chelly, J., Bober, E., Arnold, H., Ott, M. O., Gros, F., and Pinset, C. (1991) Developmental patterns in the expression of Myf5, MyoD, myogenin, and MRF4 during myogenesis. New Biol 3, 592-600

63. Asfour, H. A., Allouh, M. Z., and Said, R. S. (2018) Myogenic regulatory factors: The orchestrators of myogenesis after 30 years of discovery. Exp Biol Med (Maywood) 243, 118-128

64. Mullen, A. J., and Barton, P. J. (2000) Structural characterization of the human fast skeletal muscle troponin I gene (TNNI2). Gene 242, 313-320

65. Wei, B., and Jin, J. P. (2016) TNNT1, TNNT2, and TNNT3: Isoform genes, regulation, and structure-function relationships. Gene $\mathbf{5 8 2}, 1$-13

66. Meredith, C., Herrmann, R., Parry, C., Liyanage, K., Dye, D. E., Durling, H. J., Duff, R. M., Beckman, K., de Visser, M., van der Graaff, M. M., Hedera, P., Fink, J. K., Petty, E. M., Lamont, P., Fabian, V., Bridges, L., Voit, T., Mastaglia, F. L., and Laing, N. G. (2004) Mutations in the slow skeletal muscle fiber myosin heavy chain gene (MYH7) cause laing early-onset distal myopathy (MPD1). Am J Hum Genet 75, 703-708

67. Bjornson, C. R., Cheung, T. H., Liu, L., Tripathi, P. V., Steeper, K. M., and Rando, T. A. (2012) Notch signaling is necessary to maintain quiescence in adult muscle stem cells. Stem Cells 30, 232-242

68. Mourikis, P., and Tajbakhsh, S. (2014) Distinct contextual roles for Notch signalling in skeletal muscle stem cells. BMC Dev Biol 14, 2

69. Markert, C. D., Meaney, M. P., Voelker, K. A., Grange, R. W., Dalley, H. W., Cann, J. K., Ahmed, M., Bishwokarma, B., Walker, S. J., Yu, S. X., Brown, M., Lawlor, M. W., Beggs, A. H., and Childers, M. K. (2010) Functional muscle analysis of the Tcap knockout mouse. Hum Mol Genet 19, 2268-2283

70. Hackman, P., Vihola, A., Haravuori, H., Marchand, S., Sarparanta, J., De Seze, J., Labeit, S., Witt, C., Peltonen, L., Richard, I., and Udd, B. (2002) Tibial muscular dystrophy is a titinopathy caused by mutations in TTN, the gene encoding the giant skeletal-muscle protein titin. Am J Hum Genet 71, 492-500

71. Laurence, A., Tato, C. M., Davidson, T. S., Kanno, Y., Chen, Z., Yao, Z., Blank, R. B., Meylan, F., Siegel, R., Hennighausen, L., Shevach, E. M., and O'Shea J, J. (2007) 
Sahinyan et al. 2021 manuscript

Interleukin-2 signaling via STAT5 constrains T helper 17 cell generation. Immunity 26, 371-381

72. Buckingham, M. (1994) Muscle differentiation. Which myogenic factors make muscle? Curr Biol 4, 61-63

73. Jin, Y., Peng, Y., Lin, Z., Chen, Y. C., Wei, L., Hacker, T. A., Larsson, L., and Ge, Y. (2016) Comprehensive analysis of tropomyosin isoforms in skeletal muscles by top-down proteomics. J Muscle Res Cell Motil 37, 41-52

74. El Haddad, M., Jean, E., Turki, A., Hugon, G., Vernus, B., Bonnieu, A., Passerieux, E., Hamade, A., Mercier, J., Laoudj-Chenivesse, D., and Carnac, G. (2012) Glutathione peroxidase 3, a new retinoid target gene, is crucial for human skeletal muscle precursor cell survival. J Cell Sci 125, 6147-6156

75. Pasut, A., Oleynik, P., and Rudnicki, M. A. (2012) Isolation of muscle stem cells by fluorescence activated cell sorting cytometry. Methods Mol Biol 798, 53-64

76. Carlson, M. E., Conboy, M. J., Hsu, M., Barchas, L., Jeong, J., Agrawal, A., Mikels, A. J., Agrawal, S., Schaffer, D. V., and Conboy, I. M. (2009) Relative roles of TGF-beta1 and Wnt in the systemic regulation and aging of satellite cell responses. Aging Cell 8, 676689

77. Girardi, F., Taleb, A., Ebrahimi, M., Datye, A., Gamage, D. G., Peccate, C., Giordani, L., Millay, D. P., Gilbert, P. M., Cadot, B., and Le Grand, F. (2021) TGFbeta signaling curbs cell fusion and muscle regeneration. Nat Commun 12, 750

78. Tidball, J. G. (2011) Mechanisms of muscle injury, repair, and regeneration. Compr Physiol 1, 2029-2062

79. Relaix, F., Bencze, M., Borok, M. J., Der Vartanian, A., Gattazzo, F., Mademtzoglou, D., Perez-Diaz, S., Prola, A., Reyes-Fernandez, P. C., Rotini, A., and Taglietti, t. (2021) Perspectives on skeletal muscle stem cells. Nat Commun 12, 692

80. Gao, Q. Q., and McNally, E. M. (2015) The Dystrophin Complex: Structure, Function, and Implications for Therapy. Compr Physiol 5, 1223-1239

81. McLean, C. Y., Bristor, D., Hiller, M., Clarke, S. L., Schaar, B. T., Lowe, C. B., Wenger, A. M., and Bejerano, G. (2010) GREAT improves functional interpretation of cis-regulatory regions. Nat Biotechnol 28, 495-501

82. Sambasivan, R., Gayraud-Morel, B., Dumas, G., Cimper, C., Paisant, S., Kelly, R. G., and Tajbakhsh, S. (2009) Distinct regulatory cascades govern extraocular and pharyngeal arch muscle progenitor cell fates. Dev Cell 16, 810-821

83. Tichy, E. D., Sidibe, D. K., Greer, C. D., Oyster, N. M., Rompolas, P., Rosenthal, N. A., Blau, H. M., and Mourkioti, F. (2018) A robust Pax7EGFP mouse that enables the visualization of dynamic behaviors of muscle stem cells. Skelet Muscle 8, 27

84. Bourgey, M., Dali, R., Eveleigh, R., Chen, K. C., Letourneau, L., Fillon, J., Michaud, M., Caron, M., Sandoval, J., Lefebvre, F., Leveque, G., Mercier, E., Bujold, D., Marquis, P., Van, P. T., Anderson de Lima Morais, D., Tremblay, J., Shao, X., Henrion, E., Gonzalez, E., Quirion, P. O., Caron, B., and Bourque, G. (2019) GenPipes: an open-source framework for distributed and scalable genomic analyses. Gigascience 8

85. Bolger, A. M., Lohse, M., and Usadel, B. (2014) Trimmomatic: a flexible trimmer for Illumina sequence data. Bioinformatics 30, 2114-2120 
Sahinyan et al. 2021 manuscript

1300 86. Li, H., and Durbin, R. (2009) Fast and accurate short read alignment with BurrowsWheeler transform. Bioinformatics 25, 1754-1760

1302

87. Li, H., Handsaker, B., Wysoker, A., Fennell, T., Ruan, J., Homer, N., Marth, G., Abecasis,

1303 G., Durbin, R., and Genome Project Data Processing, S. (2009) The Sequence

1304

1305 Alignment/Map format and SAMtools. Bioinformatics 25, 2078-2079

88. Zhang, Y., Liu, T., Meyer, C. A., Eeckhoute, J., Johnson, D. S., Bernstein, B. E., Nusbaum, C., Myers, R. M., Brown, M., Li, W., and Liu, X. S. (2008) Model-based analysis of ChIPSeq (MACS). Genome Biol 9, R137

89. Quinlan, A. R. (2014) BEDTools: The Swiss-Army Tool for Genome Feature Analysis. Curr Protoc Bioinformatics 47, 1112 11-34

90. Liao, Y., Smyth, G. K., and Shi, W. (2014) featureCounts: an efficient general purpose program for assigning sequence reads to genomic features. Bioinformatics 30, 923-930

91. Love, M. I., Huber, W., and Anders, S. (2014) Moderated estimation of fold change and dispersion for RNA-seq data with DESeq2. Genome Biol 15, 550

92. Yu, G., Wang, L. G., and He, Q. Y. (2015) ChIPseeker: an R/Bioconductor package for ChIP peak annotation, comparison and visualization. Bioinformatics 31, 2382-2383

93. Ramirez, F., Dundar, F., Diehl, S., Gruning, B. A., and Manke, T. (2014) deepTools: a flexible platform for exploring deep-sequencing data. Nucleic Acids Res 42, W187-191

94. Stark R, B. G. (2011) DiffBind: differential binding analysis of ChIP-Seq peak data. in Bioconductor

95. Robinson, M. D., McCarthy, D. J., and Smyth, G. K. (2010) edgeR: a Bioconductor package for differential expression analysis of digital gene expression data.

Bioinformatics 26, 139-140

96. Heinz, S., Benner, C., Spann, N., Bertolino, E., Lin, Y. C., Laslo, P., Cheng, J. X., Murre, C., Singh, H., and Glass, C. K. (2010) Simple combinations of lineage-determining transcription factors prime cis-regulatory elements required for macrophage and $B$ cell identities. Mol Cell 38, 576-589

97. Korotkevich, G., Sukhov, V., Budin, N., Shpak, B., Artyomov, M. N., and Sergushichev, A. (2021) Fast gene set enrichment analysis. bioRxiv, 060012 
Figure 1

1. Myofiber

isolation

2. Individual myofiber transfered to tube, lysed with water and permeabilized with $0.5 \%$ Triton-X

3. DNA tagmentation and adaptor incorporation

4. Column purification
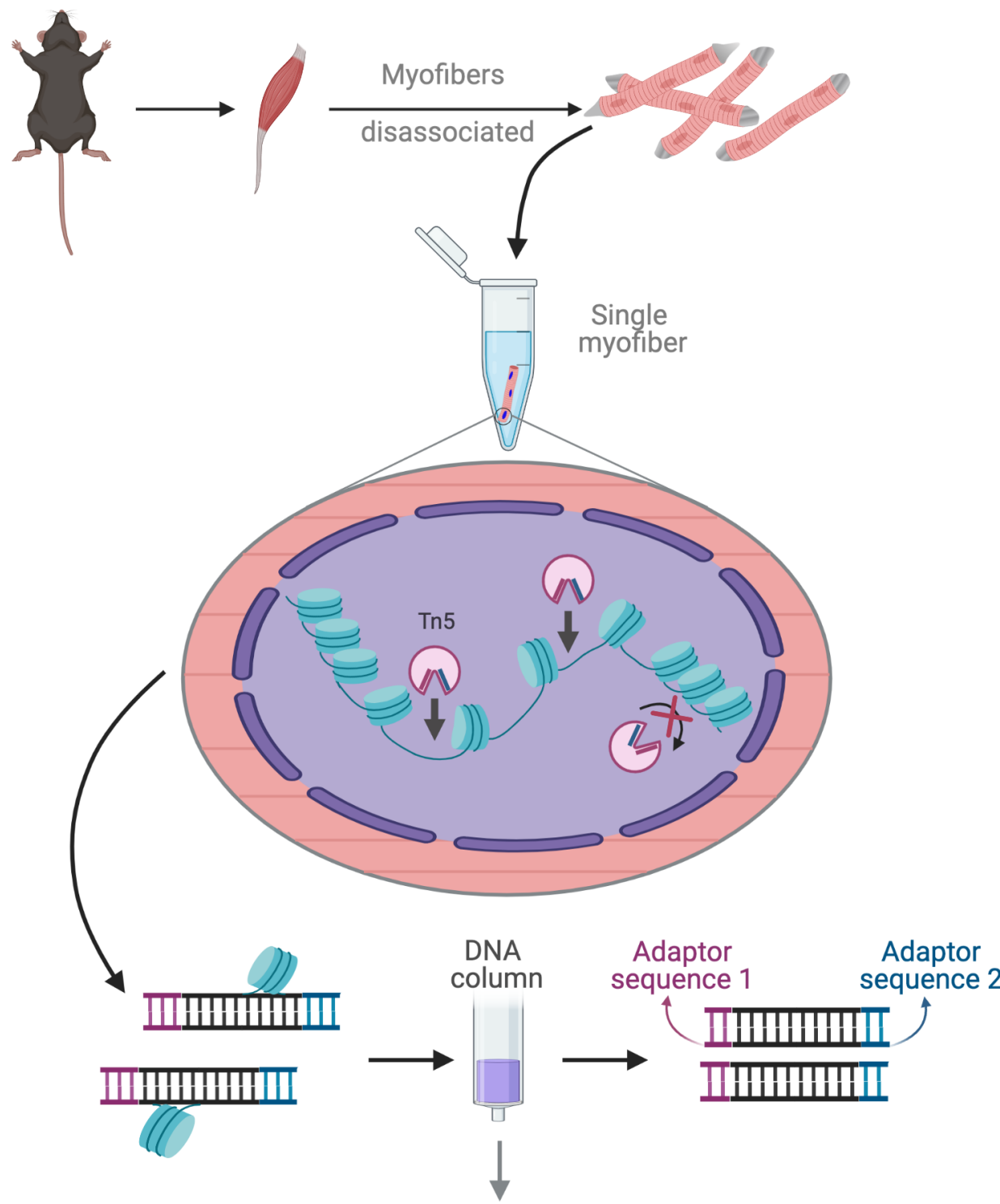

5. PCR amplification and library preperation

6. AMPure bead size selelction and purification

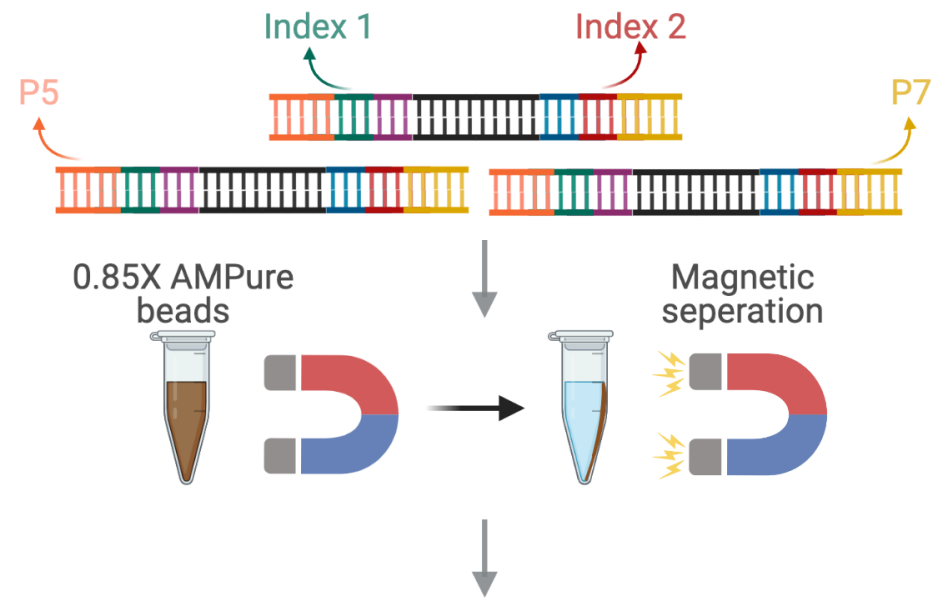

Sequence 
Figure 2

A Uninjured fiber

B

Injured fiber
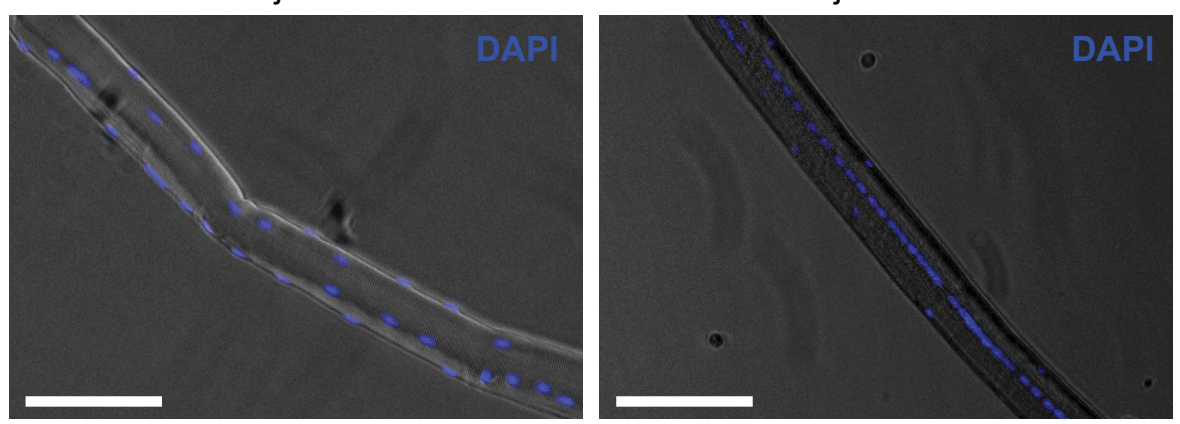

C MuSCs

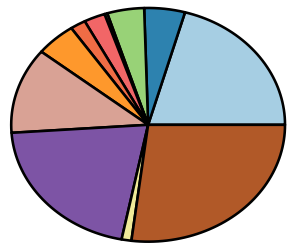

D Injured fibers

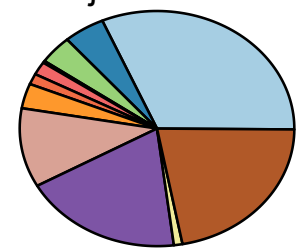

E Uninjured fibers

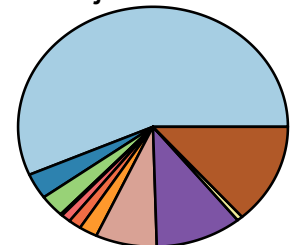

Promoter (+/- 1kb TSS)

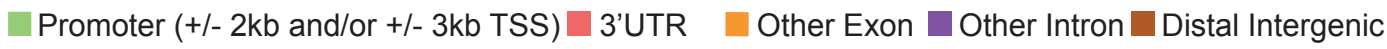

a Promoter (+/- 1kb and/or +/- 2kb TSS) ['UTR

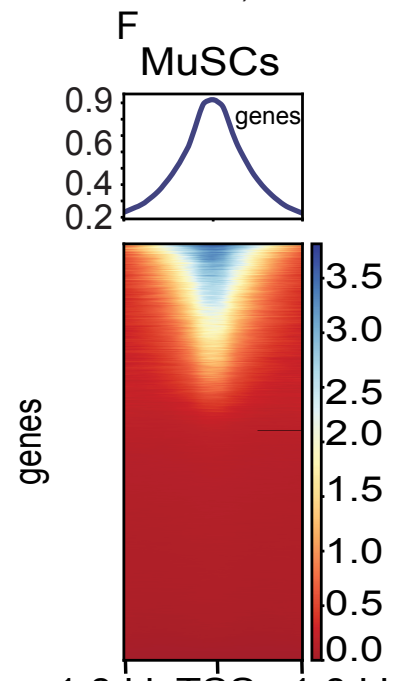

$-1.0 \mathrm{~kb}$ TSS $+1.0 \mathrm{~kb}$

15 Uninjured fib фqr

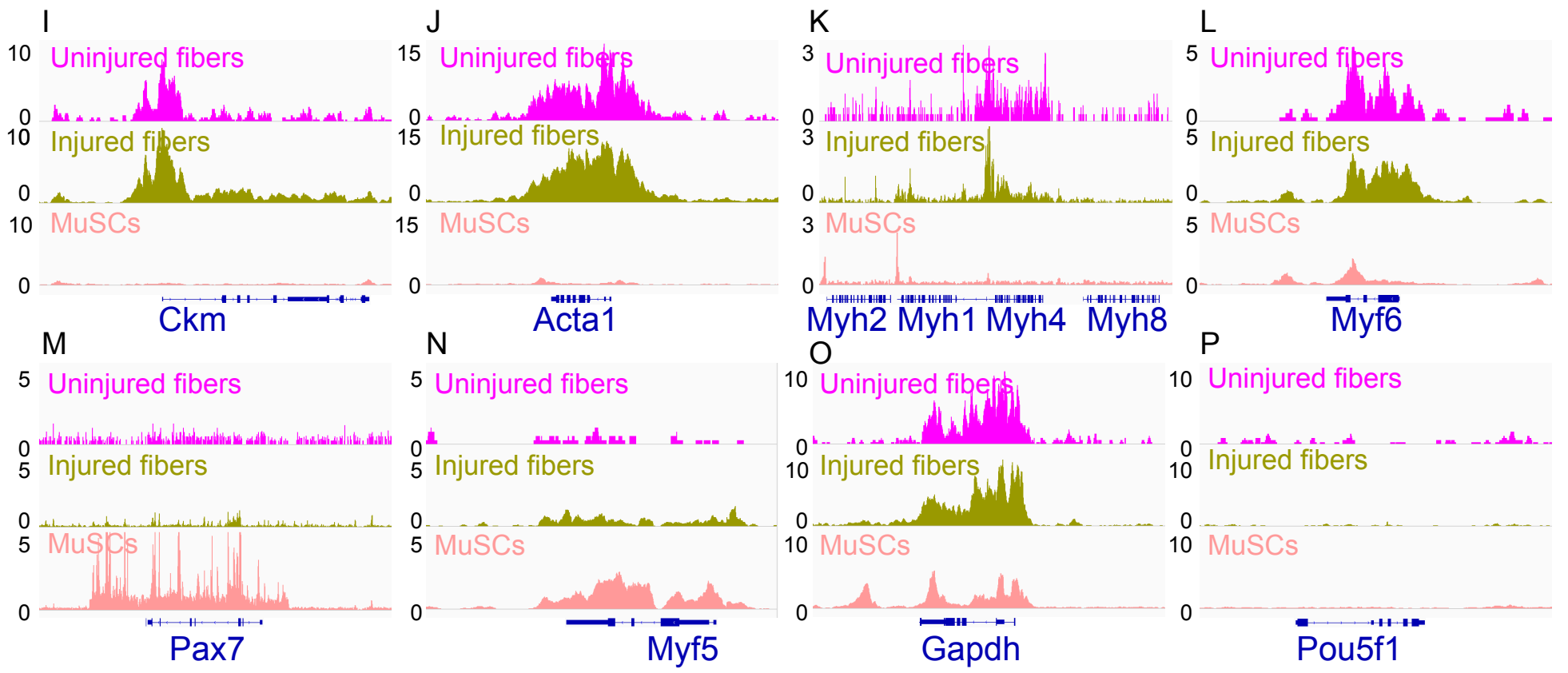

G

Injured fibers
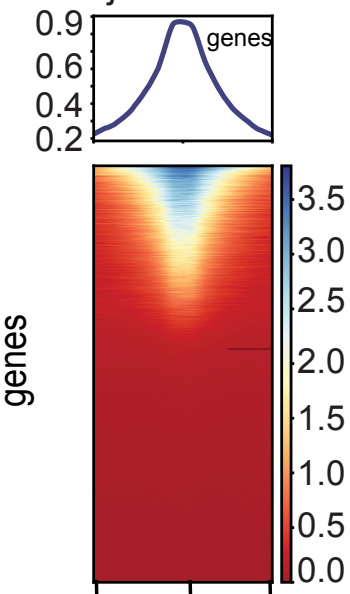

$-1.0 \mathrm{~kb}$ TSS +1.0 kb

1st Exon $\square$ 1st Intron Downstream (<=300 kb)

$\mathrm{H}$

Uninjured fibers
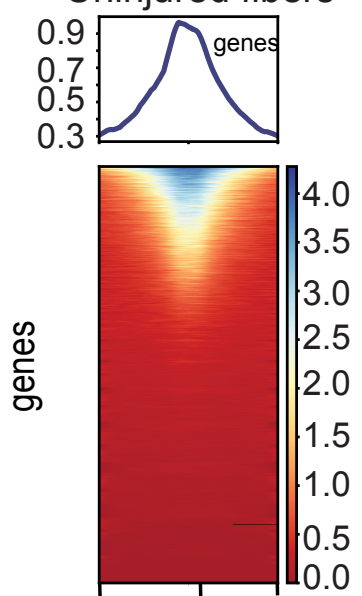

$-1.0 \mathrm{~kb}$ TSS $+1.0 \mathrm{~kb}$ 
Figure 3
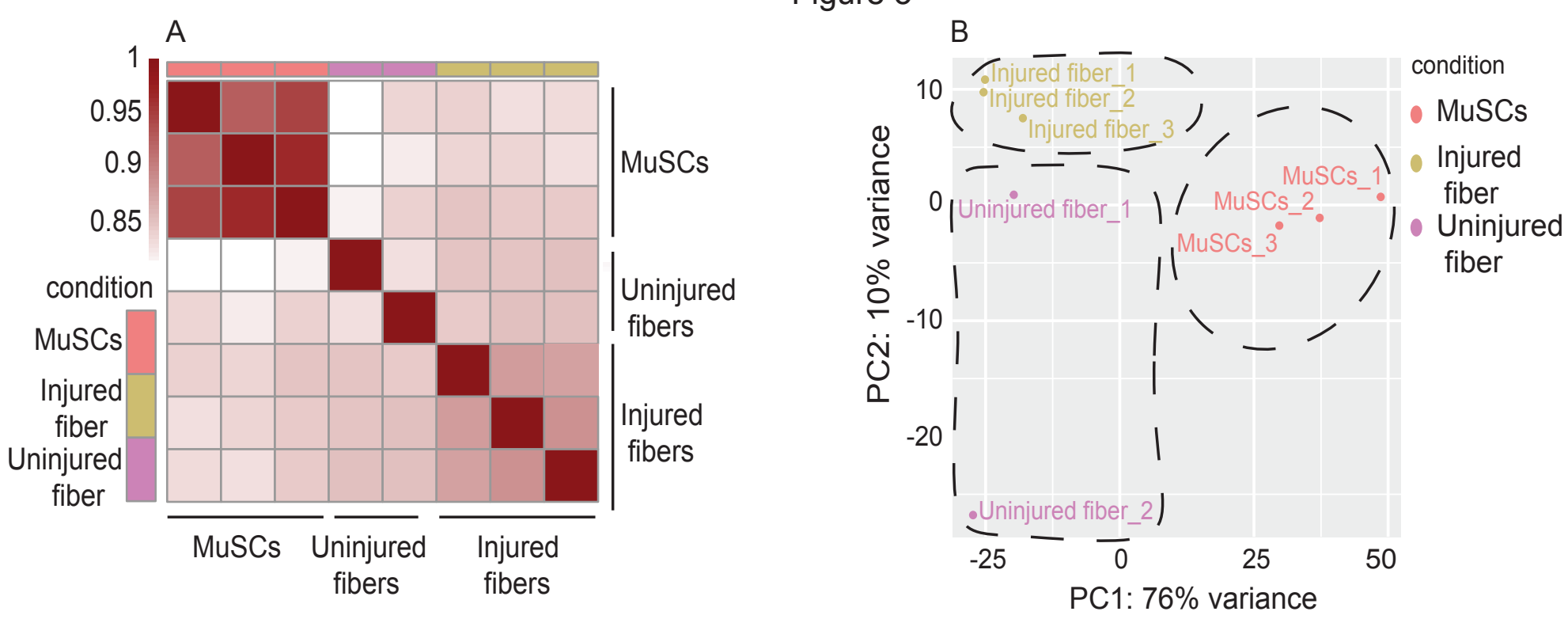

C

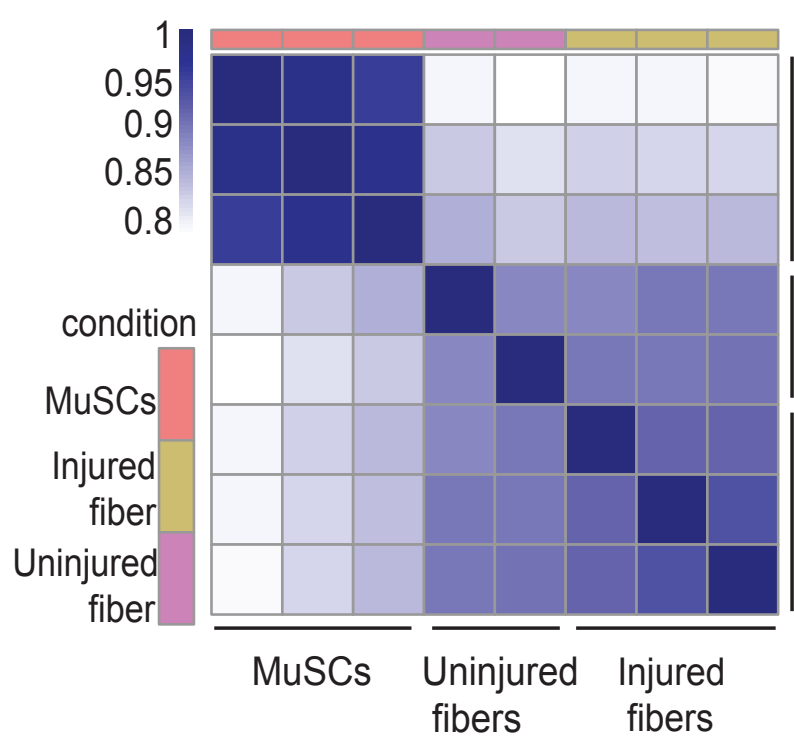

E

Differential peaks

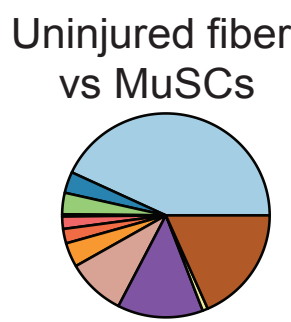

Promoter (+/- $1 \mathrm{~kb}$ TSS)
Uninjured fiber vs Injured fiber

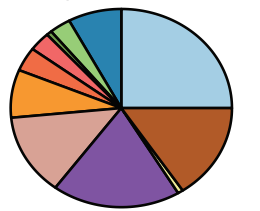

Promoter (+/- $2 \mathrm{~kb}$ and/or +/-3 kb TSS)

5'UTR

ather Intron

Downstream (<=300 kb)

1st Exon
D
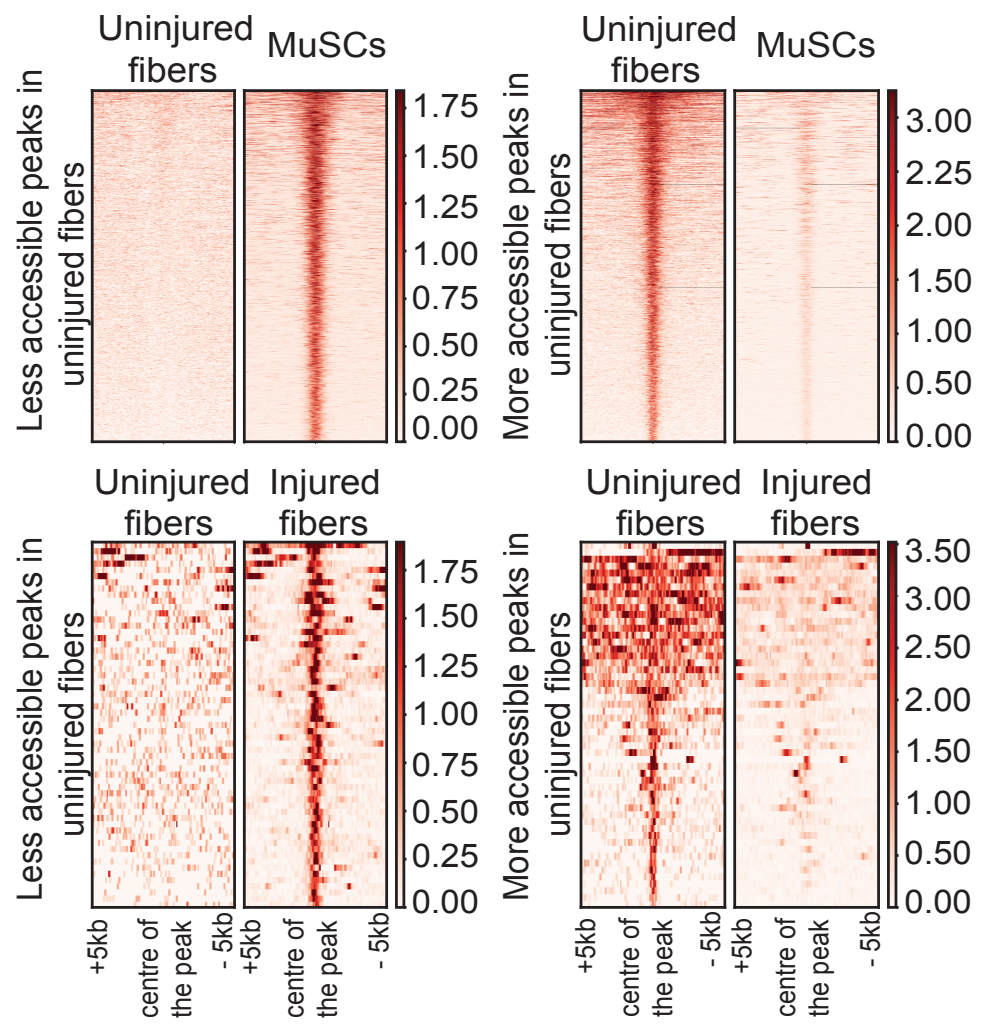

F Number of ATAC-Seq peaks

Uninjured MuSCs

Uninjured Injured fibers
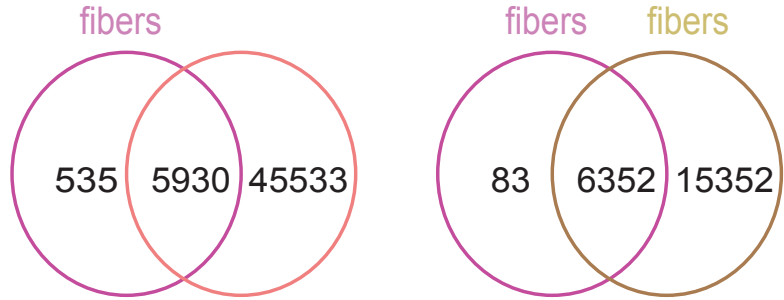

1st Intron

3'UTR

Distal Intergenic 


\section{Figure 4}

A

\section{Uninjured fibers \\ GO Biological Process \\ $-\log 10$ (Binomial $p$ value)}

$\begin{array}{llll}0 & 20 & 40 & 60\end{array}$

Striated muscle cell development Muscle cell development Actomyosin structure organization Component assembly in morphogenesis

Cardiac muscle cell development

Cardiac cell development

Regulation of metabolites and energy

Polll transcription in response to stress mRNA catabolic process

Transcription in response to stress

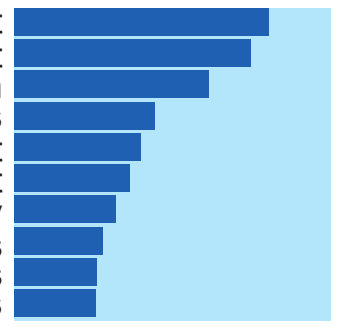

B

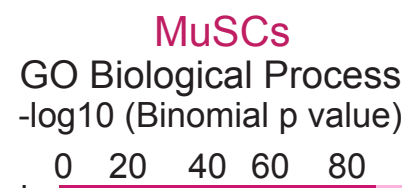

Response to incorrect protein

$\begin{array}{lllll}0 & 20 & 40 & 60 & 80\end{array}$

Mitochondrial membrane permeability
ER unfolded protein response

Cellular response to unfolded protein

Adherens junction organization

Regulation of membrane permeability

Regulation of TLR signalling pathway

Mitochondrial transmembrane transport

Execution phase of apoptosis

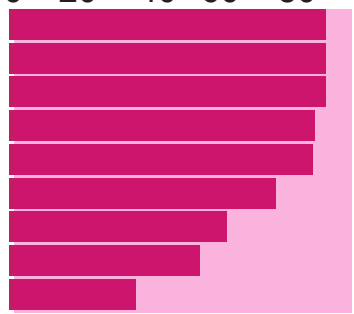

C

Injured fibers

GO Biological Process - $\log 10$ (Binomial $p$ value)

$\begin{array}{llll}0 & 40 & 80 & 120\end{array}$

Striated muscle cell development Myofibril assembly

Sarcomere organization

Muscle cell development

Actomyosin structure organization

Component assembly in morphogenesis Mitochondrial transport

Muscle fiber development

Regulation of metabolites and energy

Cardiac muscle cell development
D

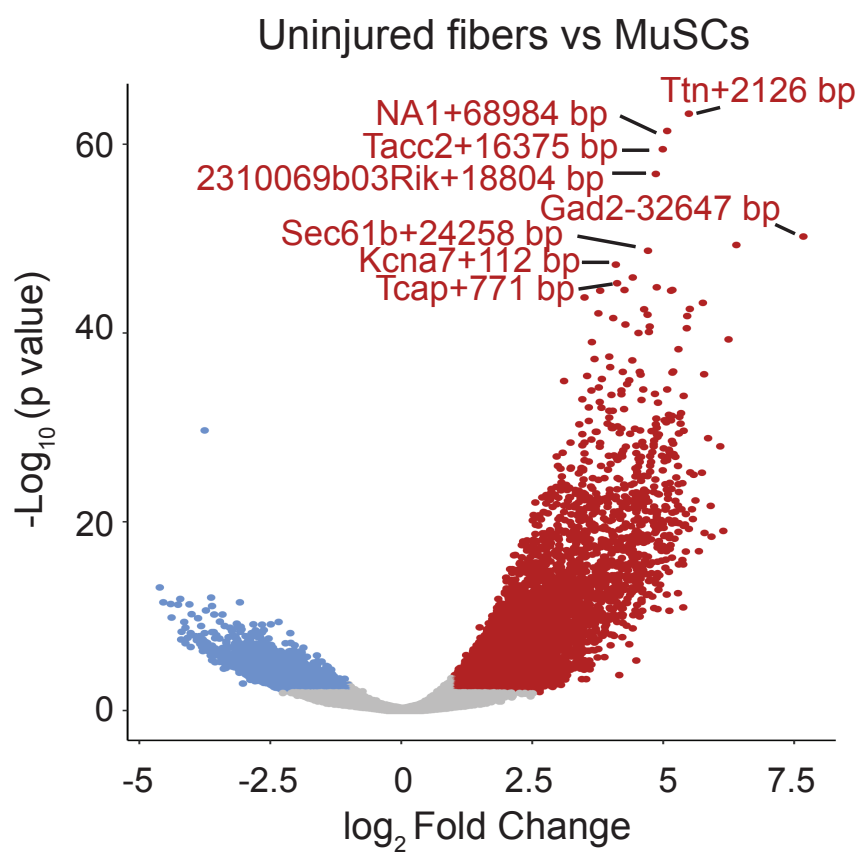

E

Injured fibers vs Uninjured fibers

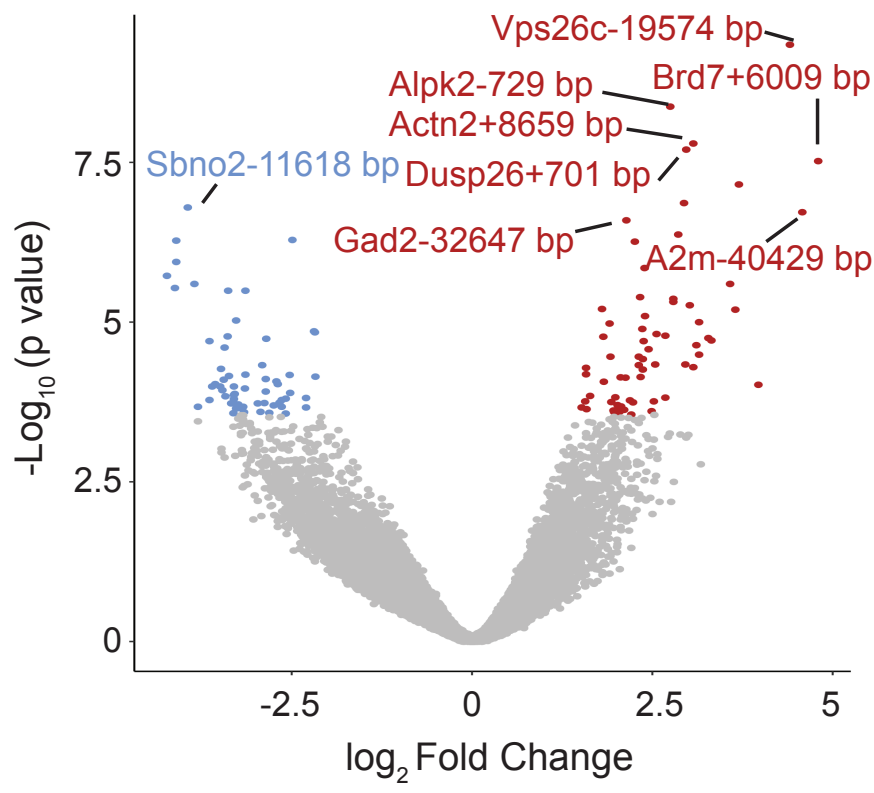


Figure 5

A

\section{Uninjured fibers vs Injured fibers}

Fatty acid metabolism Oxidative phosphorylation EMT IFNy response Myogenesis

Complement Early estrogen response

Coagulation Inflammatory response IL2-Stat5 signalling

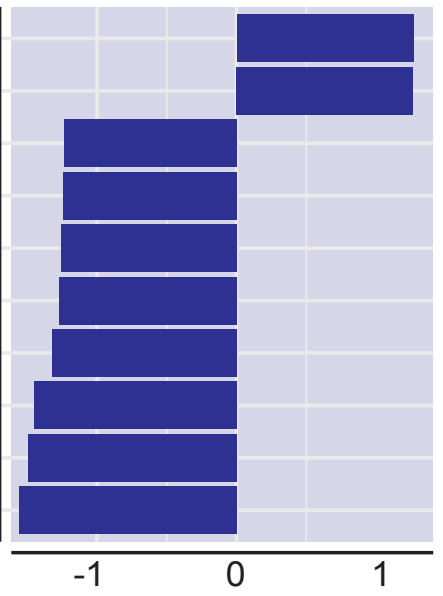

Normalized Enrichment Score

B

\section{Uninjured fibers vs MuSCs}

Oxidative phosphorylation

Myogenesis
sphorylation Peroxisome

Glycolysis

TNF- $\alpha$ signalling via NF-KB

Adipogenesis

Fatty acid metabolism

UV Response

EMT

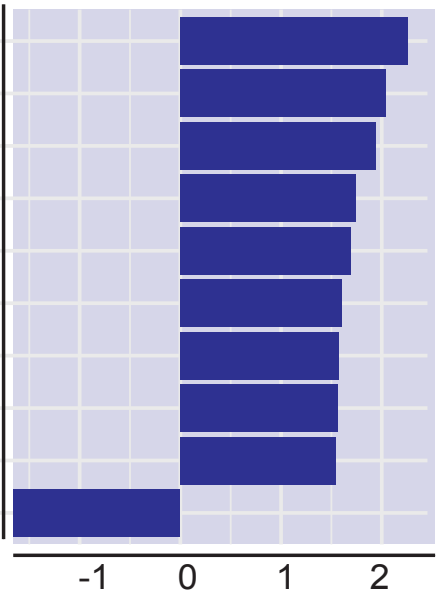

Normalized Enrichment Score
C
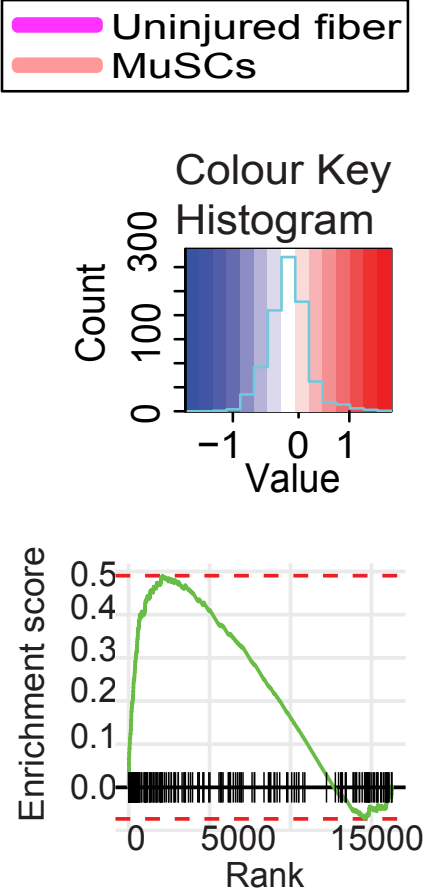

\section{D}

15 Uninjured fibers
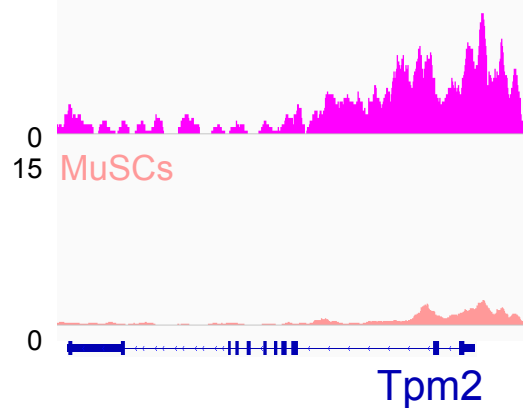

E

${ }^{8}$ Uninjured fibers
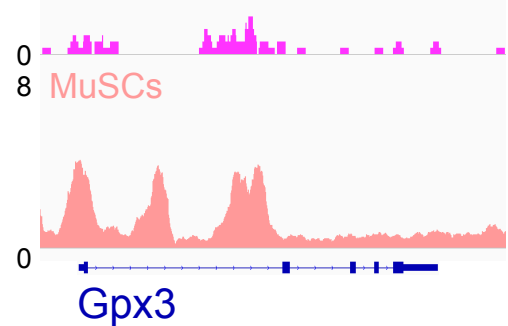

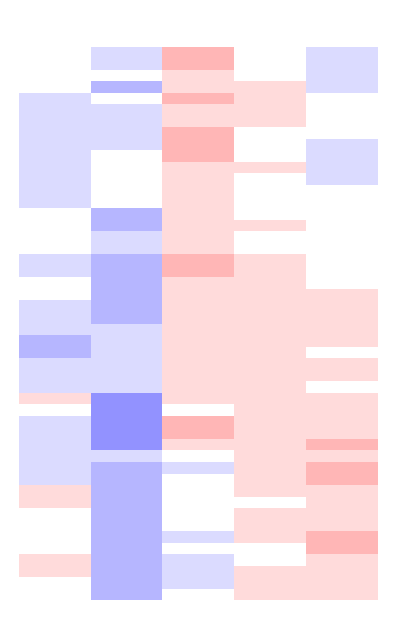

Camk2b

Ccel1

Chr

Bag1

Chrna1

Plxnb2

Fdps

Col6a2

Ablim

yl2

Mapre3 
A
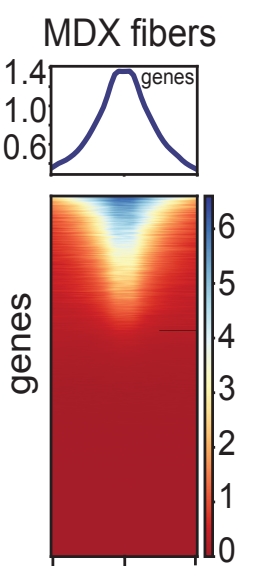

WT fibers

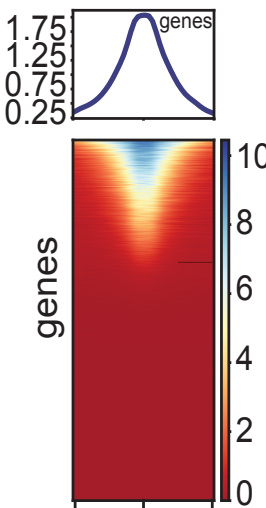

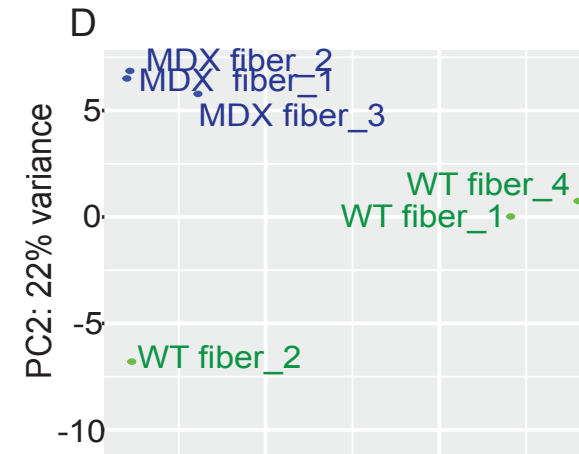

.WT fiber_3

$$
0 \quad 10
$$

PC1: $48 \%$ variance

E
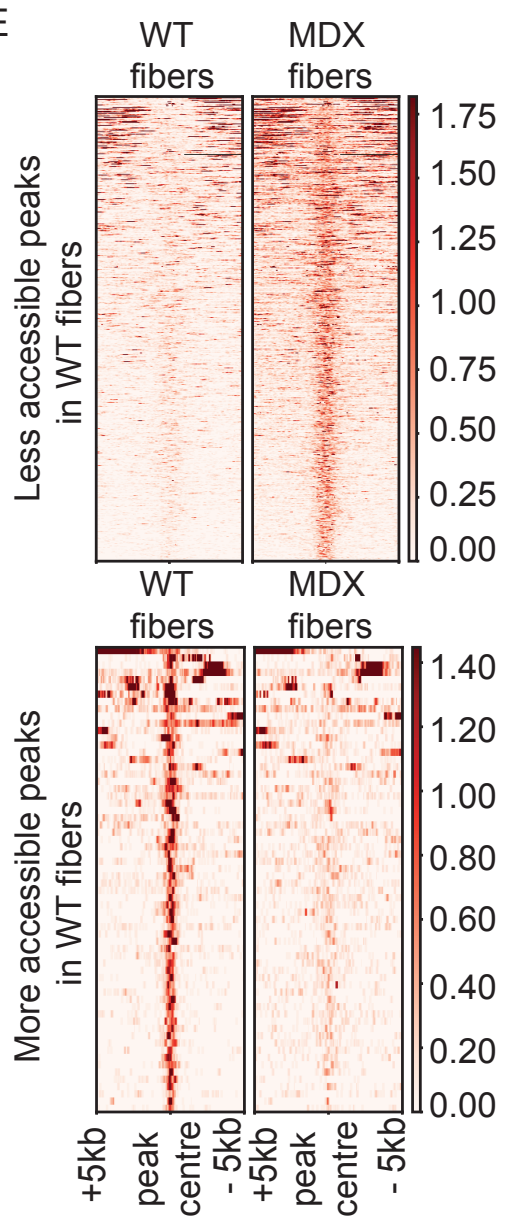

B

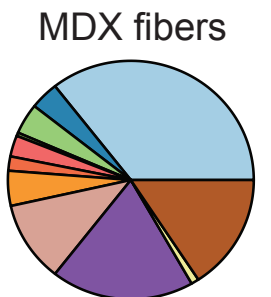

Promoter (+/- 1kb TSS)

Promoter (+/- 1kb and/or
3'UTR +/-2kbTSS)

1st Exon

Other Intron

Downstream (<=300 kb)

Figure 6

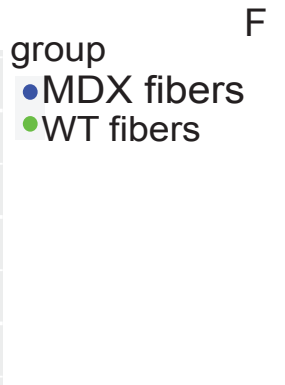

$\mathrm{F}$
Differential Peaks WT vs MDX fibers

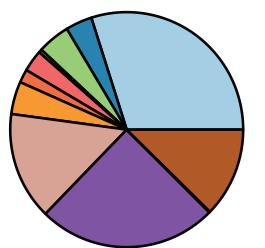

Promoter (+/- 1kb TSS) Promoter (+/- $2 \mathrm{~kb}$ and/or $+/-3 \mathrm{kbTSS})$

\section{Promoter (+/- $1 \mathrm{~kb}$ and/or \\ Other Exon $+/-2 \mathrm{kbTSS})$}

1st Intron

Other Intron

Downstream (<=300 kb)

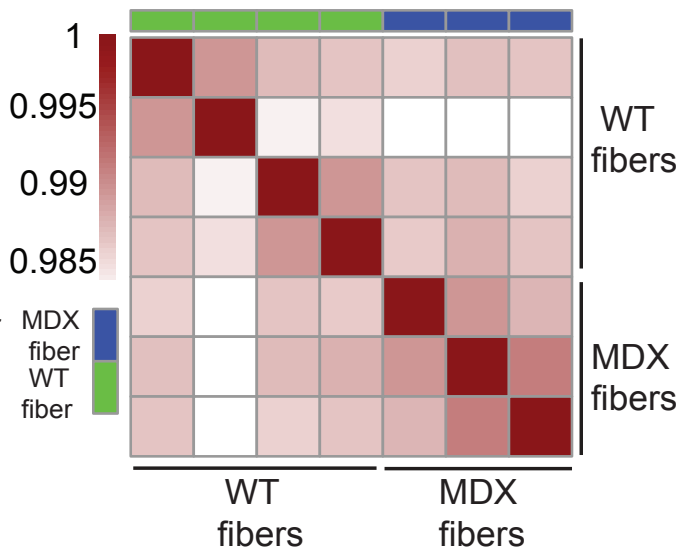

G

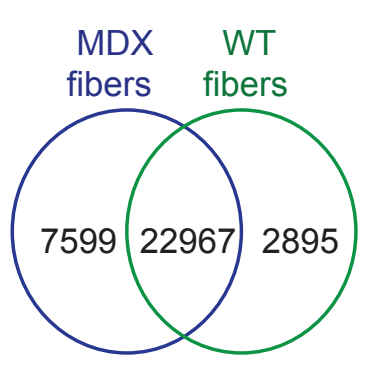

3'UTR

1st Exon

Distal Intergenic

Peaks unique to

H MDX fibers GO Biological Process $-\log 10$ (Binomial $p$ value)

actin cystoskeleton organization Striated muscle cell differentiation regulation of calcium-release channel activity posttranscriptional regulation of gene expression regulation of translation

regulation of cellular amide metabolic process regulation of striated muscle tissue development regulation of muscle organ development Striated muscle cell development regulation of muscle tissue development

\section{Peaks unique to WT fibers \\ GO Biological Process $-\log 10$ (Binomial $p$ value)}

cellular response to biotic stimulus $\begin{array}{llllll}0 & 4 & 8 & 12 & 16 & 20\end{array}$ response to molecule of bacterial origin regulation of metabolites and energy cellular response to lipopolysaccharide cellular carbohydrate metabolic process regulation of carbohydrate metabolic process striated muscle cell development muscle cell development regulation of fatty acid oxidation cellular hormone metabolic process 
Figure 1- Figure Supplement 1

A

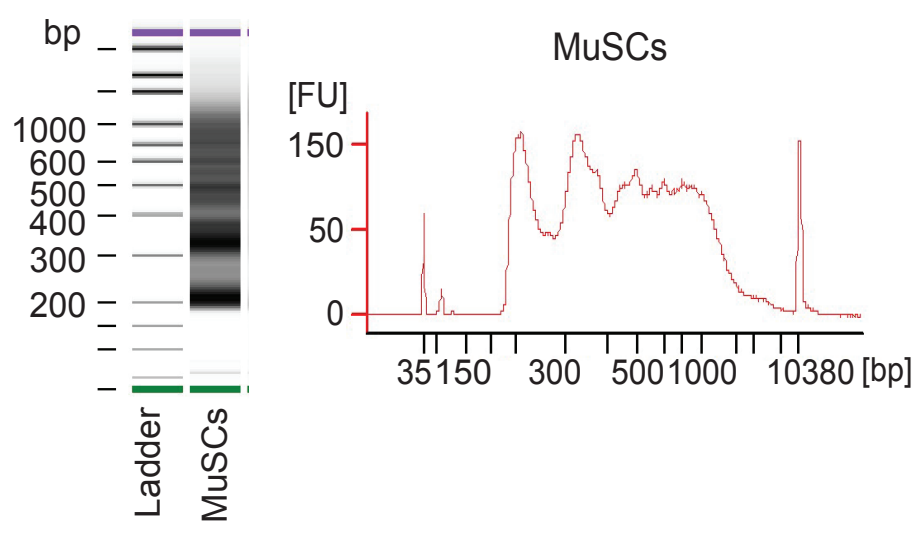

C

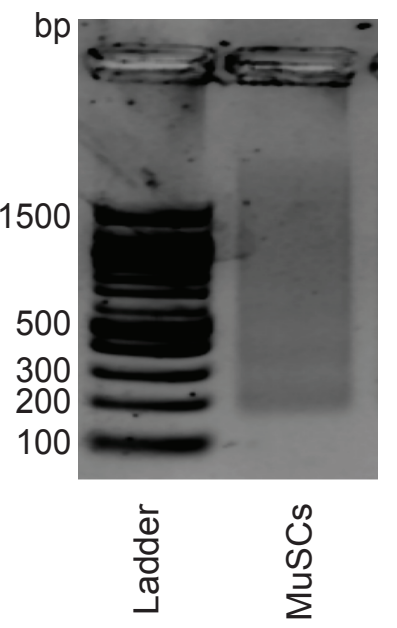

D

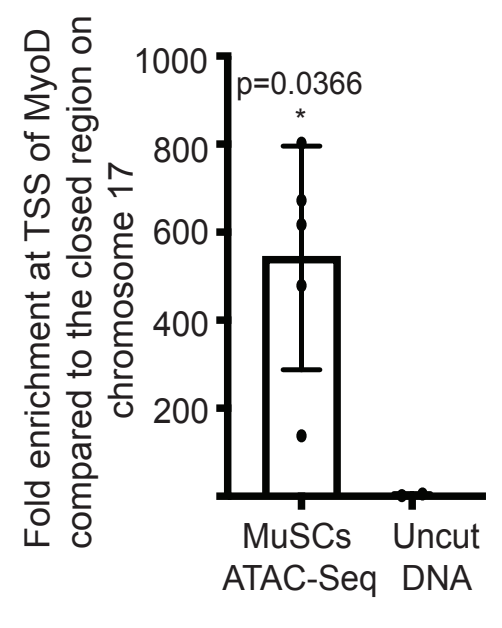

B

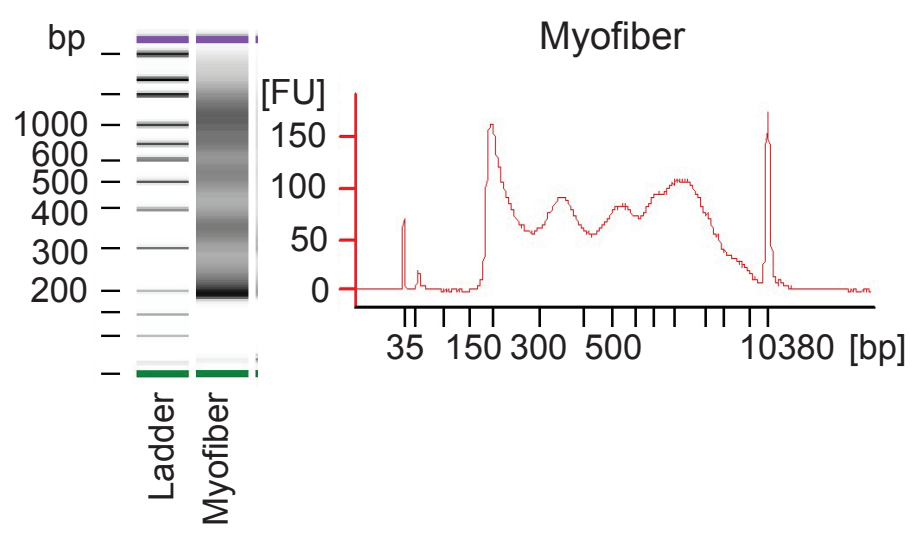

E

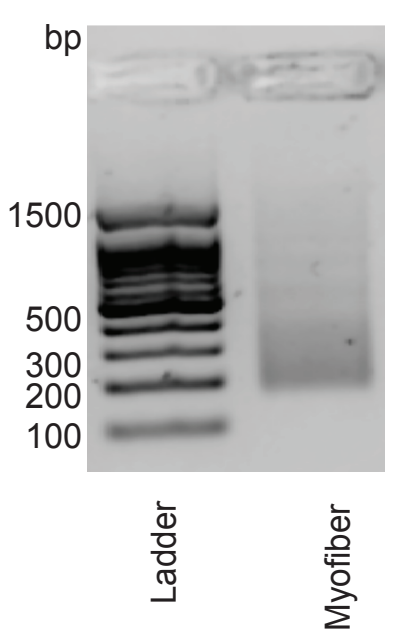

F

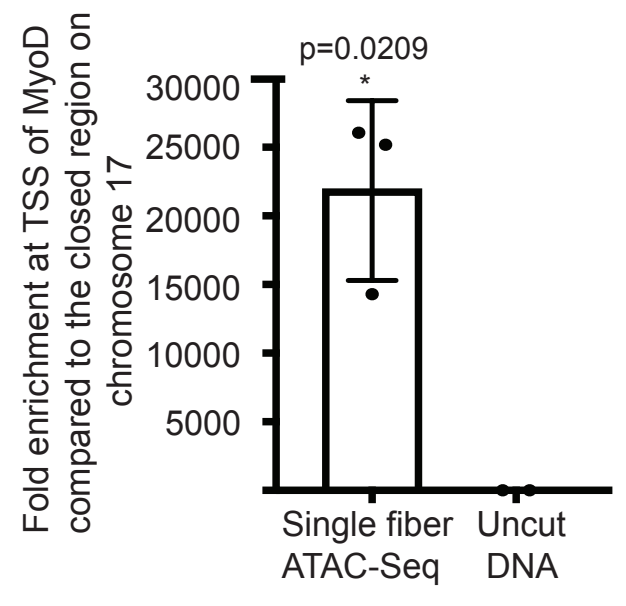


Figure 2- Figure Supplement 1

\section{A}

10 Uninjured fibers

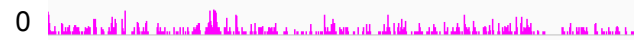

10 Injured fibers

10 MusCs

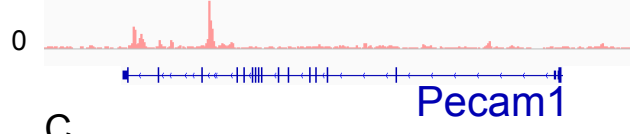

10 Uninjured fibers

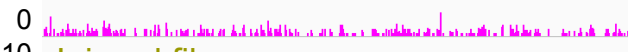

10 Injured fibers

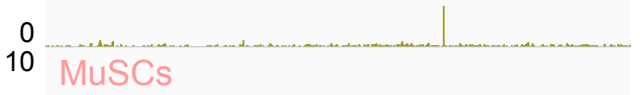

0

10 Uninjured fibers

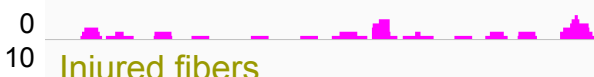

10 MuSCs

0

\section{G}

10 Uninjured fibers

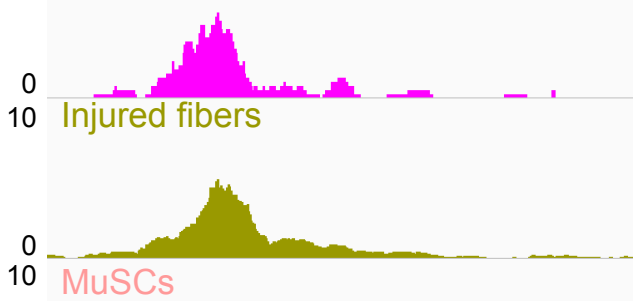

0

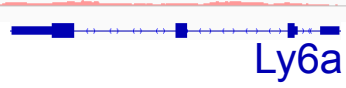

Ly6a

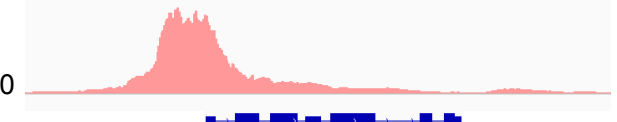

Rps2
B

10 Uninjured fibers

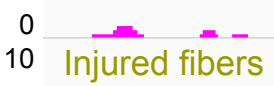

10 MuSCs

0

D

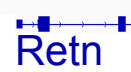

10 Uninjured fibers
10

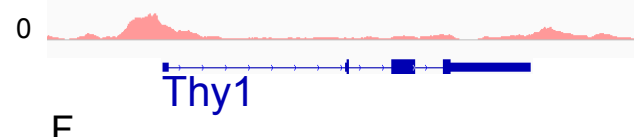

10 Uninjured fibers

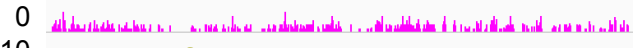

10 Injured fibers

10 MuSCs

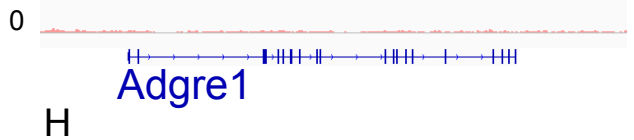

5 Uninjured fibers

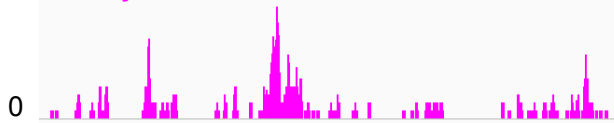
5 Injured fibers

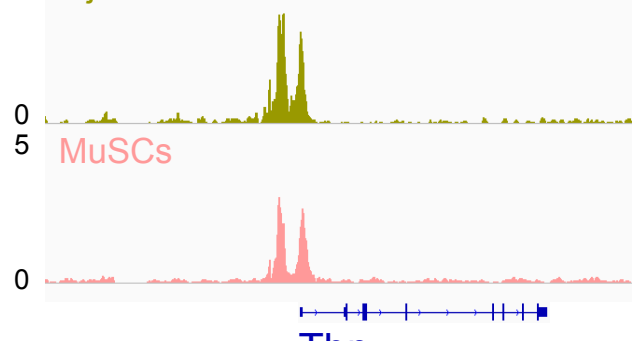

Tbp 
Figure 2- Figure Supplement 2

A

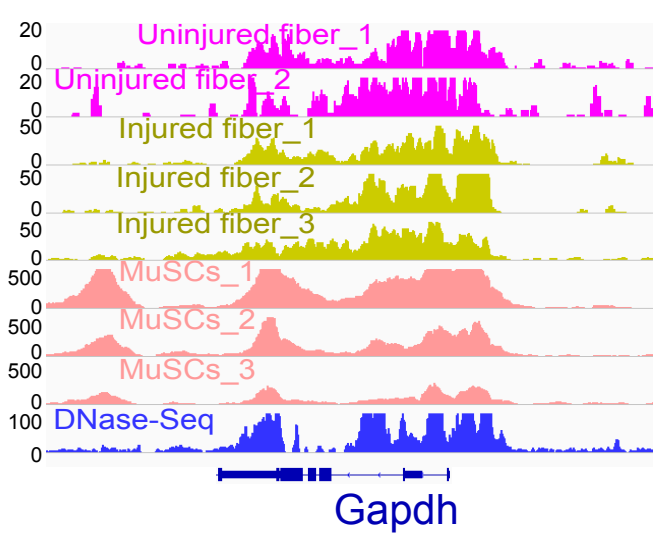

D

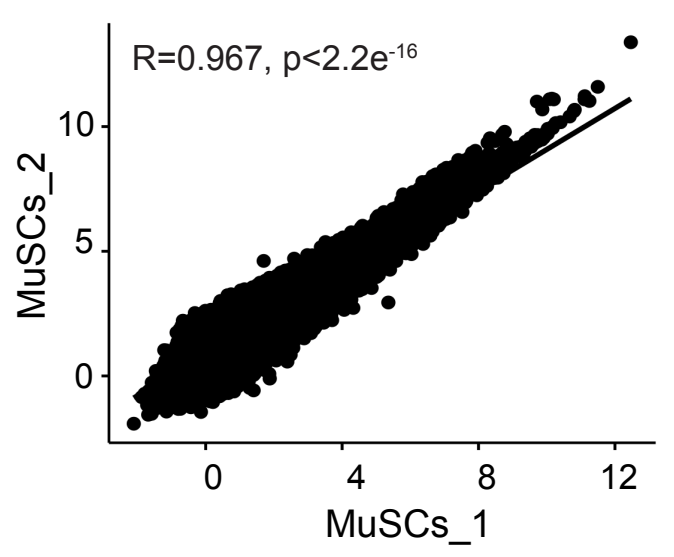

G

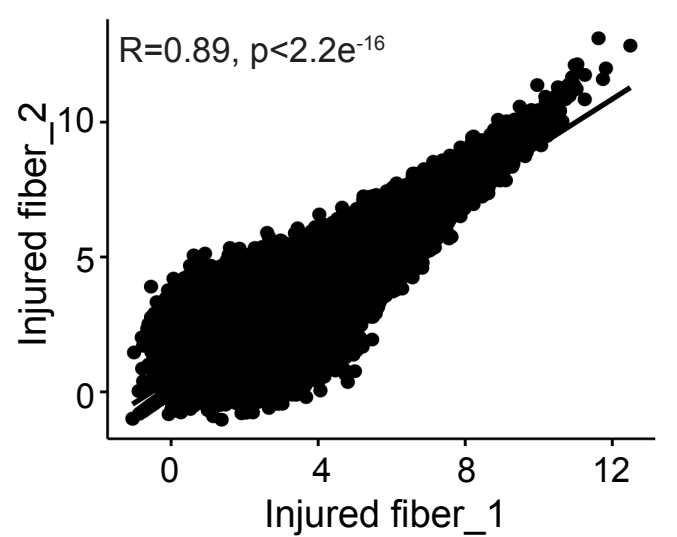

J

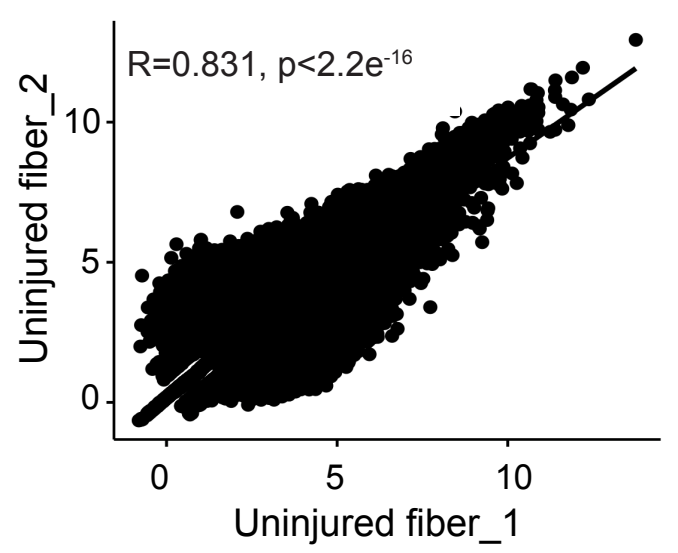

B

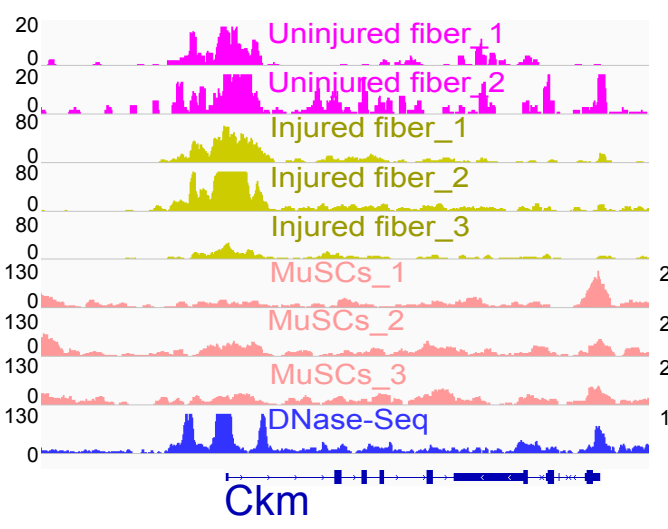

E

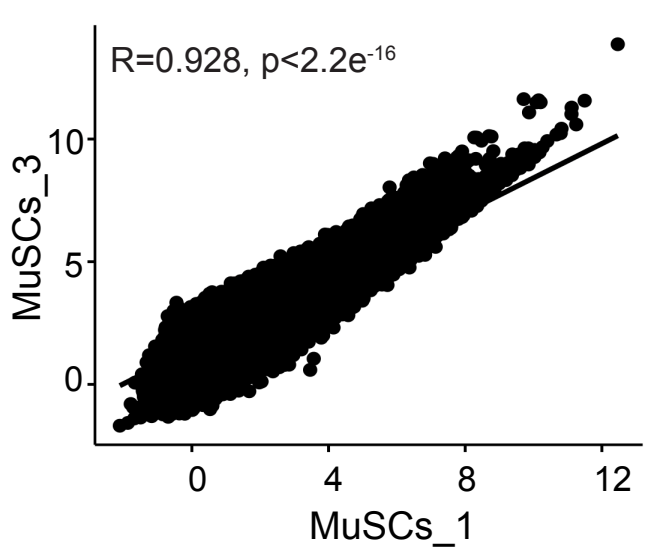

$\mathrm{H}$

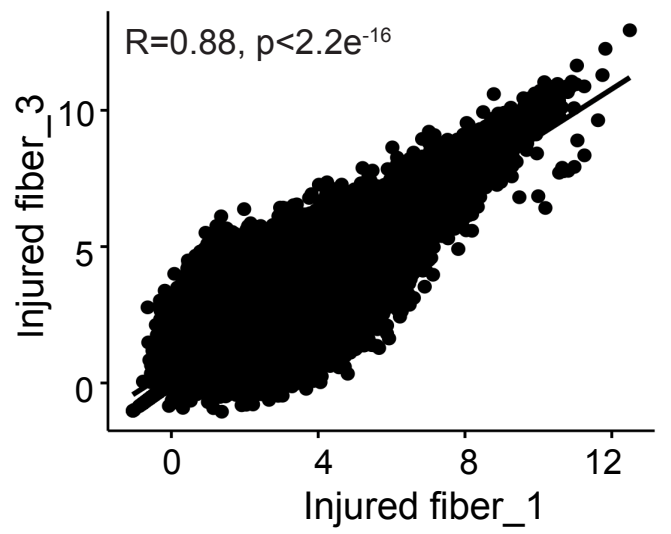

C

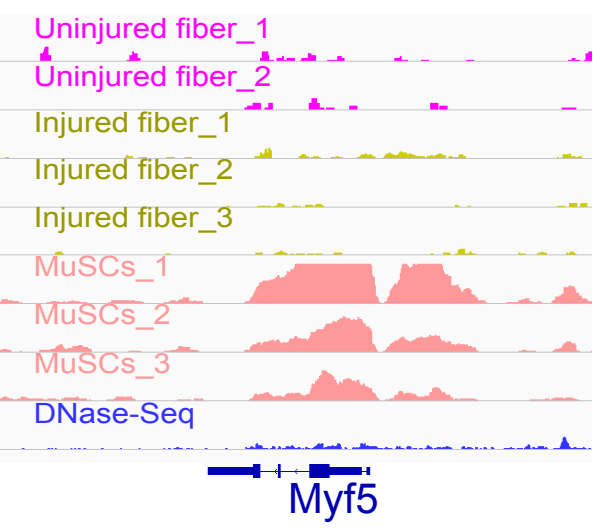

F

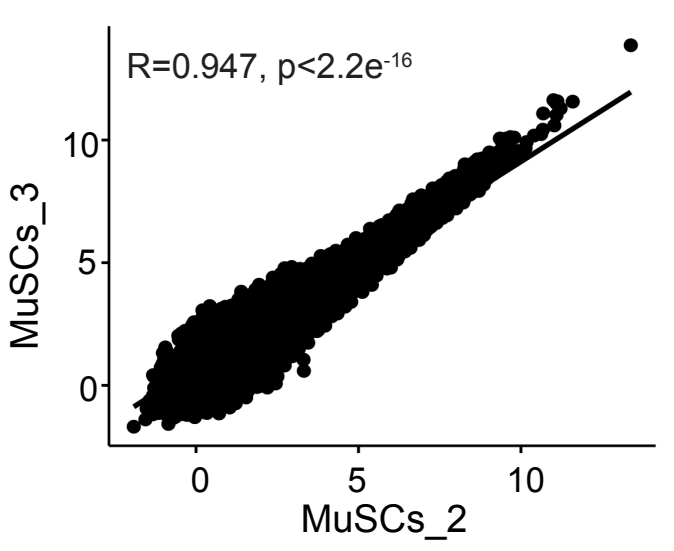

I

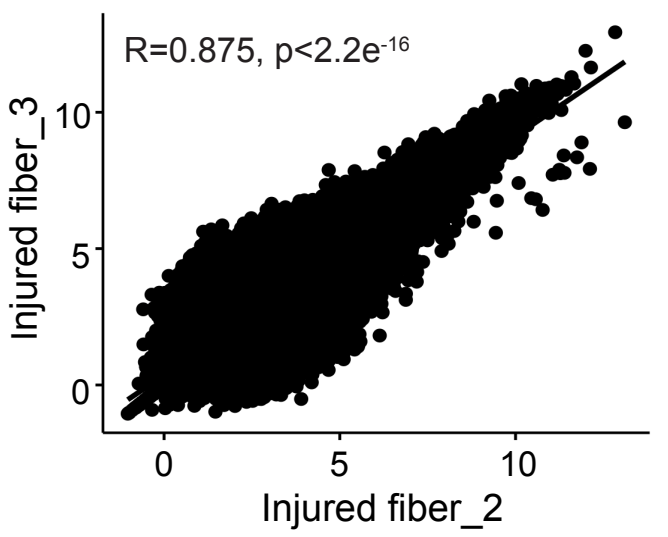

K

Number of Peaks

smfATAC-Seq EDL muscle

Uninjured EDL myofibers H3K27ac ChIP-Seq

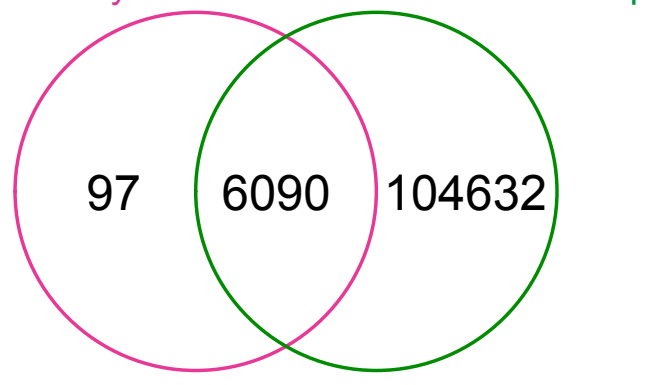


Figure 2- Figure Supplement 3

A

10 Uninjured Fibers

10 Injured Fibers

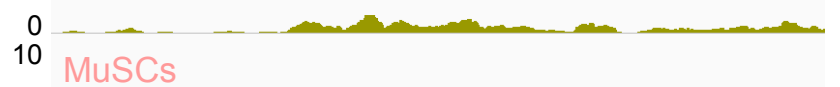

0

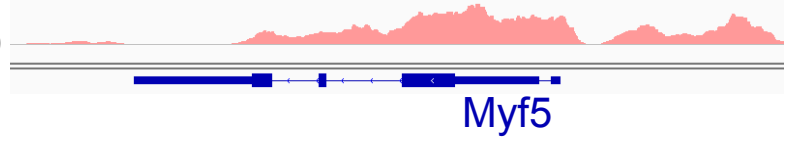

C

10 Uninjured Fibers

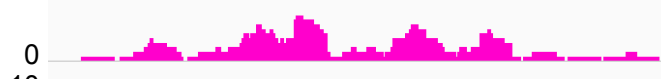

10 Injured Fibers

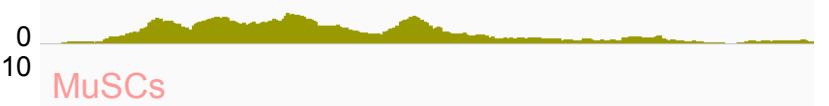

0

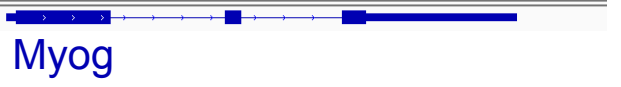

B

10 Uninjured Fibers
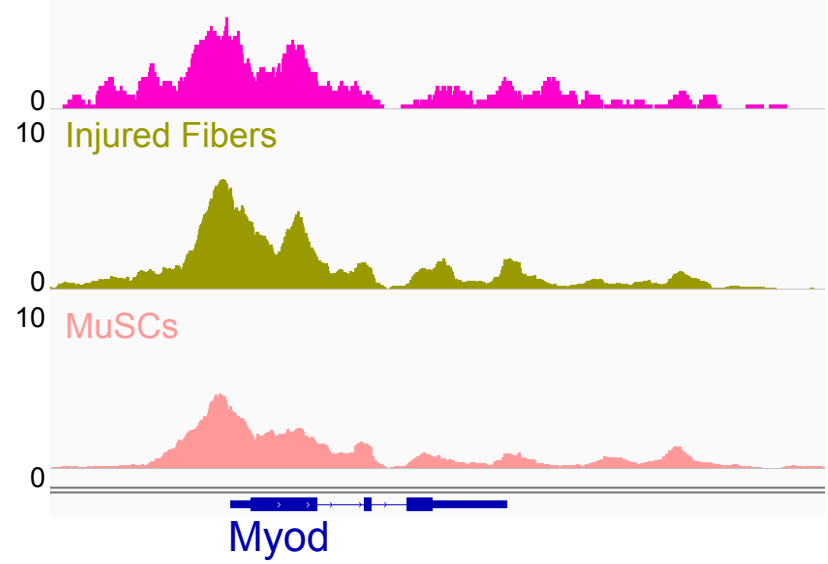

D

10 Uninjured Fibers
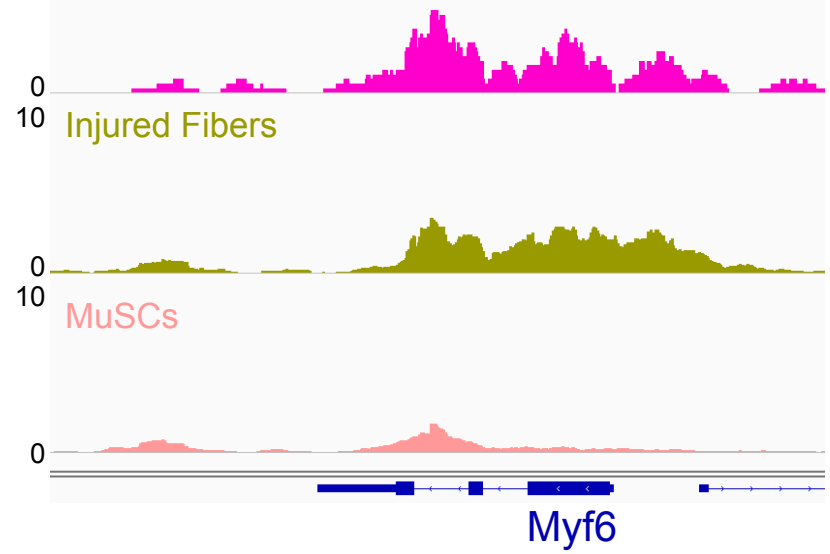
Figure 3 - Figure Supplement 1

A

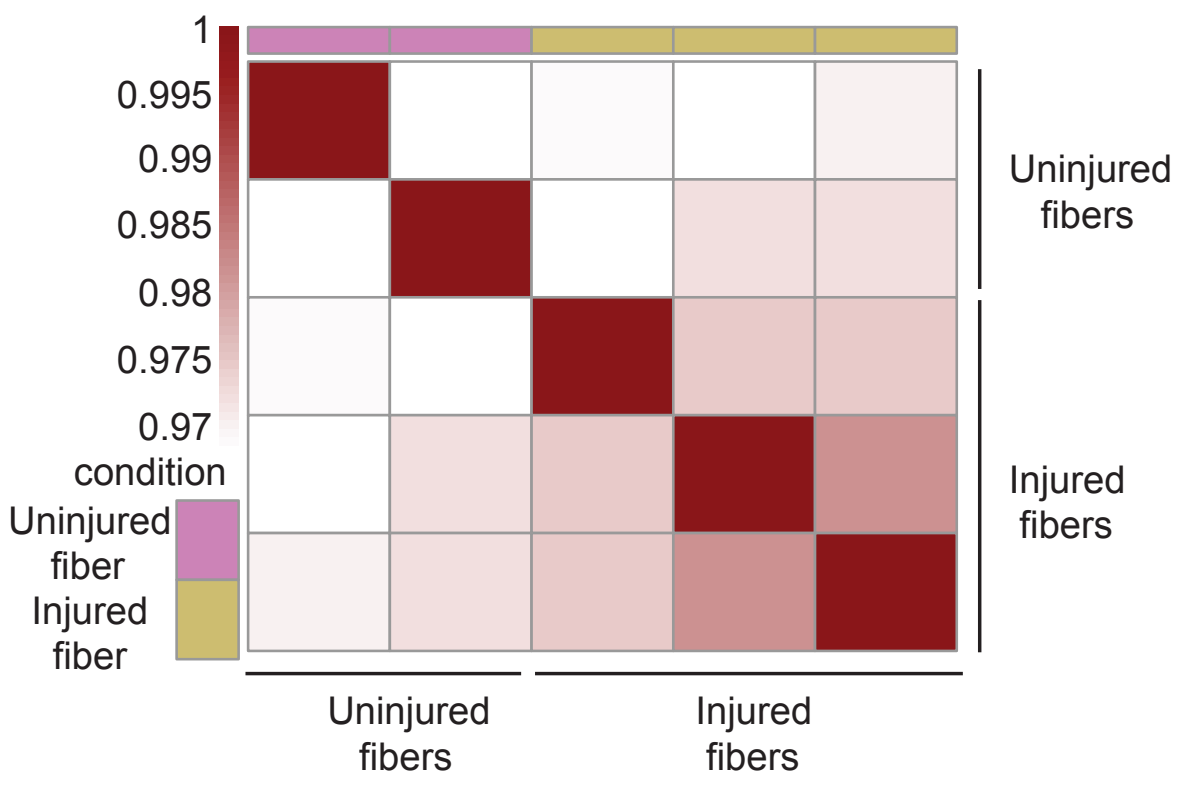

B

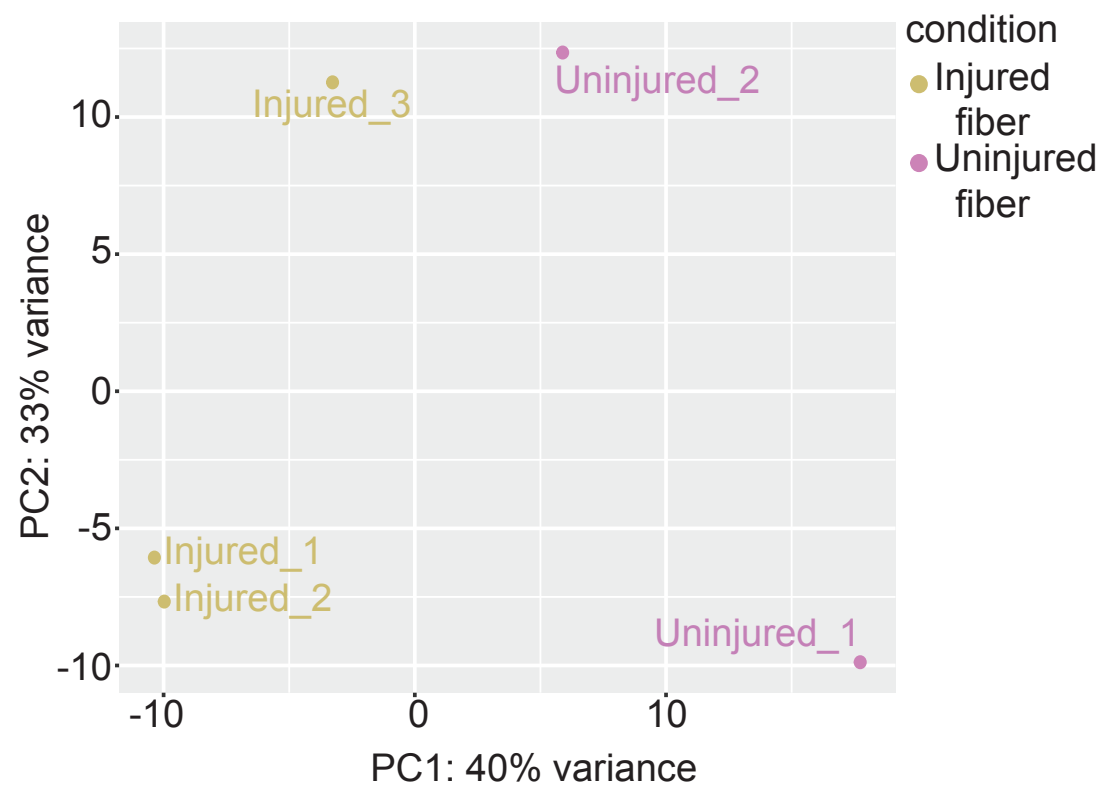


Figure 3- Figure Supplement 2

A

10

Uninjured fibers

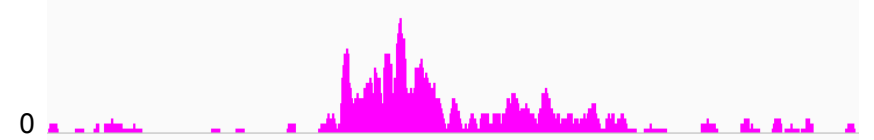

10 Injured fibers

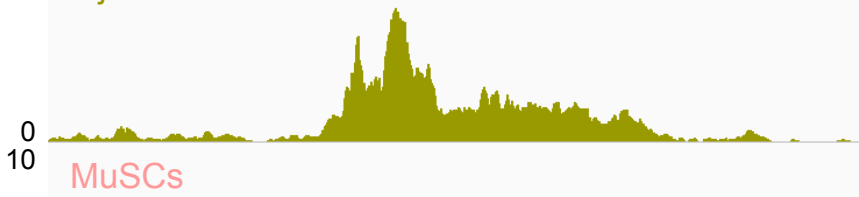

0

\section{-}

Tnni2

C

Uninjured fibers

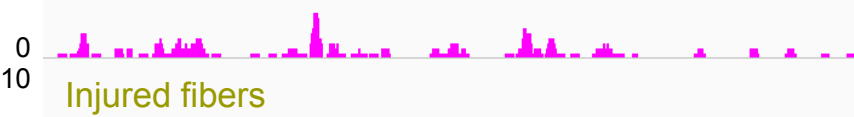

10

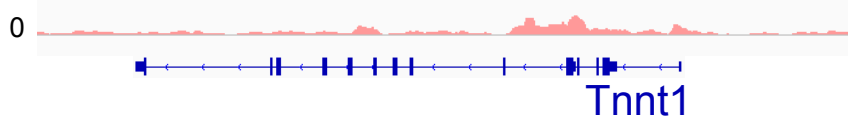

B

8

Uninjured fibers

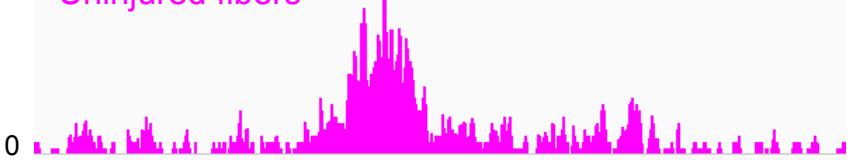

8 Injured fibers
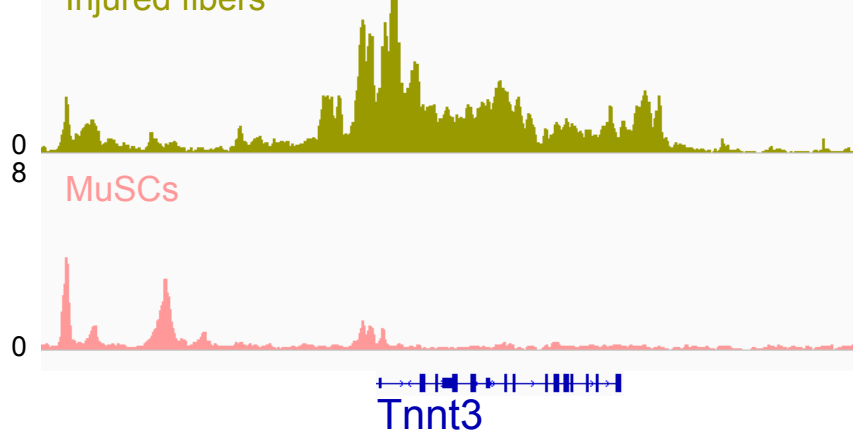

10

Uninjured fibers

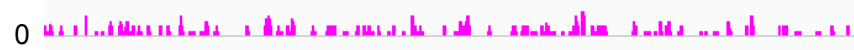
10 Injured fibers

10 MuSCs

H"W

Myh7 
Figure 3 - Figure Supplement 3
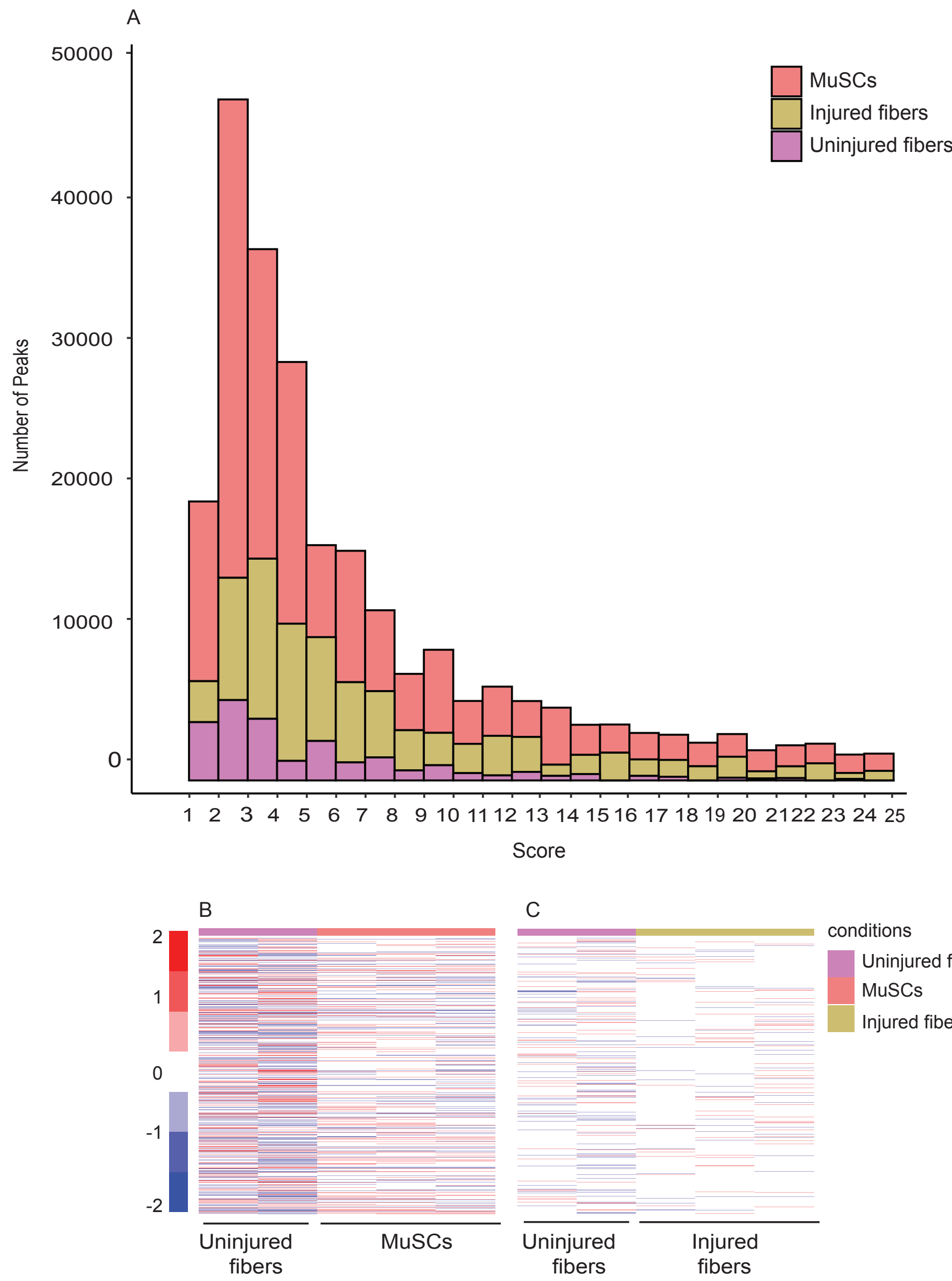

conditions

Uninjured fibers MuSCs Injured fibers 
A

Figure 4- Figure Supplement 1

Peaks unique to uninjured fibers compared to MuSCs

GO Biological Process $-\log 10$ (Binomial $p$ value)

Sarcomere organization Myofibril assembly

Striated muscle cell development

Muscle cell development Actomyosin structure organization

Cardiac muscle fiber development

Muscle thin filament assembly

Striated muscle cell differentiation

Assembly in morphogenesis

Muscle fiber development

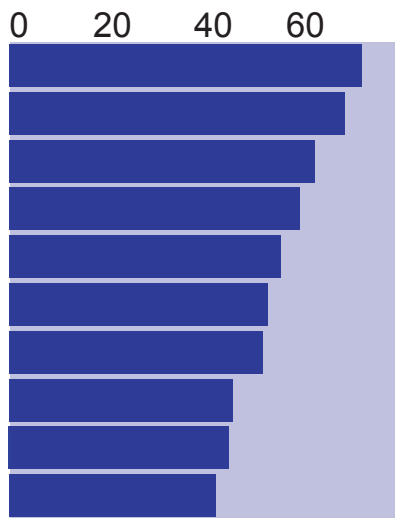

C

Peaks common to MuSCs

and uninjured fibers

GO Biological Process

- $\log 10$ (Binomial p value)

Mitochondrial transport

Pol II transcription in stress

Pol II transcription in ER stress

Pol II transcription regulation

Response to incorrect protein

Cell response to incorrect protein

Transcription in response to stress

Regulation of metabolites and energy

Mitochondrial membrane organization Protein folding

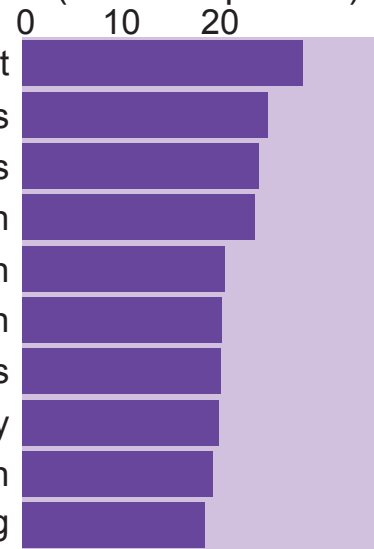

B

Peaks unique to MuSCs compared to uninjured fibers

GO Biological Process $-\log 10$ (Binomial $p$ value)

Adherens junction organization Regulation of myotube differentiation Mitochondrial membrane permeability Regulation of Notch signalling Regulation of membrane permeability Regulation of cytochrome c frelease Regulation of ERBB signalling pathway

Regulation of collagen metabolism Unsaturated fatty acid biosynthesis Regulation of collagen biosynthesis

\section{$\begin{array}{lllll}0 & 20 & 40 & 60 & 80\end{array}$}

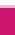

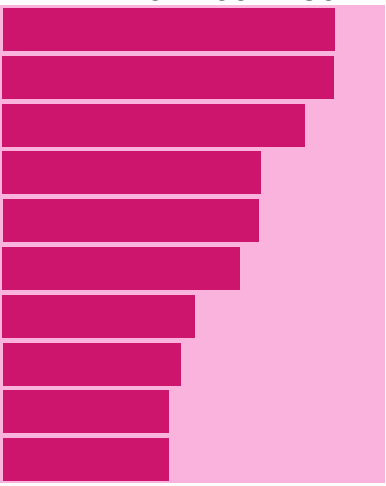

\title{
Peaks common to injured and uninjured fibers GO Biological Process - $\log 10$ (Binomial p value)
}

\author{
Myofibril assembly \\ Sarcomere organization \\ Striated muscle cell development \\ Actomyosin structure organization \\ Muscle cell development \\ Mitochondrial transport \\ Muscle fiber development \\ Assembly in morphogenesis \\ Nucleosome assembly \\ Nucleosome organization
}

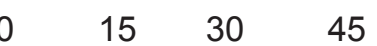

\section{E}

Peaks unique to injured fibers compared to uninjured fibers

GO Biological Process $-\log 10$ (Binomial $p$ value)

0

Striated muscle cell development

Myofibril assembly

Muscle cell development

Actomyosin structure organization

Sarcomere organization

Assembly in morphogenesis Regulation of metabolites and energy

Muscle fiber development

Cardiac cell development

Cardiac muscle celld development

$\begin{array}{llll}0 & 40 & 80 & 110\end{array}$

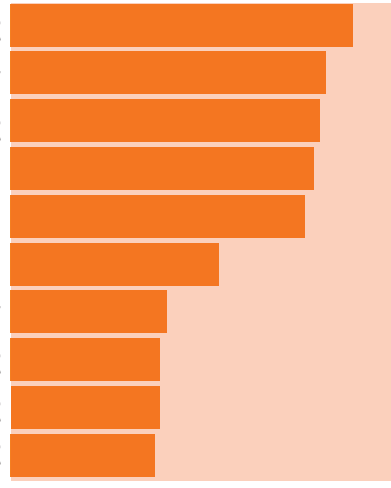

$\mathrm{F}$

Peaks unique to uninjured fibers compared to injured fibers

GO Biological Process

$-\log 10$ (Binomial $p$ value)

Myofibril assembly

Pos.regulation of $\mathrm{K}^{-}$transporter Nephron tubule formation

Fru-2,6- $\mathrm{P}_{2}$ metabolism Chitin catabolic process

Neg.regulation of K- transporter Regulation of synaptic potentiation

Alanine transport

Aminoglycan catabolic process Cytokine production in inflammation

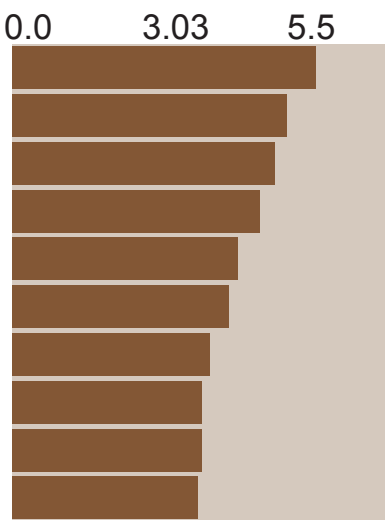


Figure 4 - Figure Supplement 2

A

Top motifs enriched in peaks common to Injured and Uninjured Myofibers overlapping the promoters

Motif

GCCCCCCCCCCC Sp1 $\mathrm{e}^{-91}$

ACTACAATTCCC GFY $\mathrm{e}^{-62}$

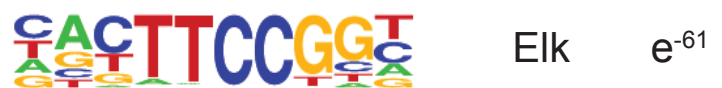

CTAAAAATAG Mef2a $\mathrm{e}^{-60}$

AACCGGAAGT ELF $\mathrm{e}^{-56}$

AACCGGAAGT ETS $\mathrm{e}^{54}$

CTCCCCATCCCC NRF $\mathrm{e}^{46}$

CE⿱ ÇCTGACGTCAC CRE $\mathrm{e}^{-30}$

CAAGATGCCGCC YY1 $\mathrm{e}^{-29}$

AECCAATCGG NFY $\mathrm{e}^{26}$
B

Top motifs enriched in peaks unique to Injured myofibers overlapping the promoters

Motif

ICCTGACTCA MafA $e^{-1561}$

ATGACTCATC JUN $\mathrm{e}^{-1513}$

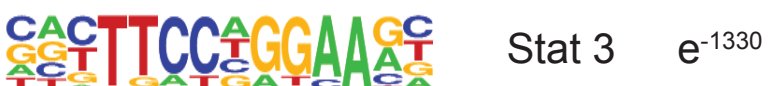

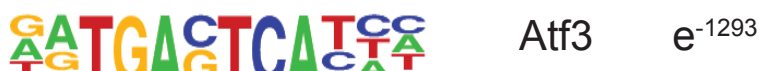

PATGACTCAT BATF $\mathrm{e}^{-1247}$

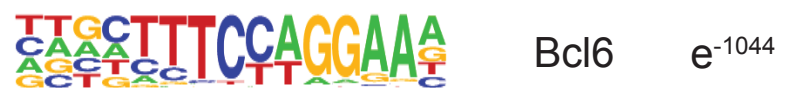

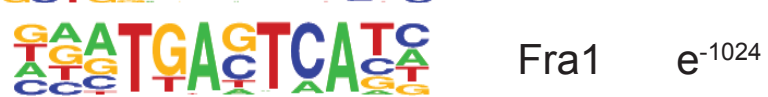

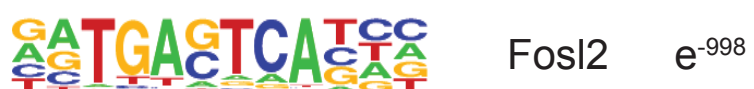

GTCATAAAA

GCCCCCCCCCCC Sp1 $\mathrm{e}^{-877}$ 
Figure 5-Figure Supplement 1

A

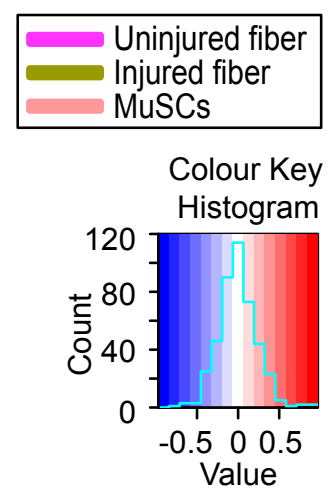

\section{Notch Signalling Pathway}

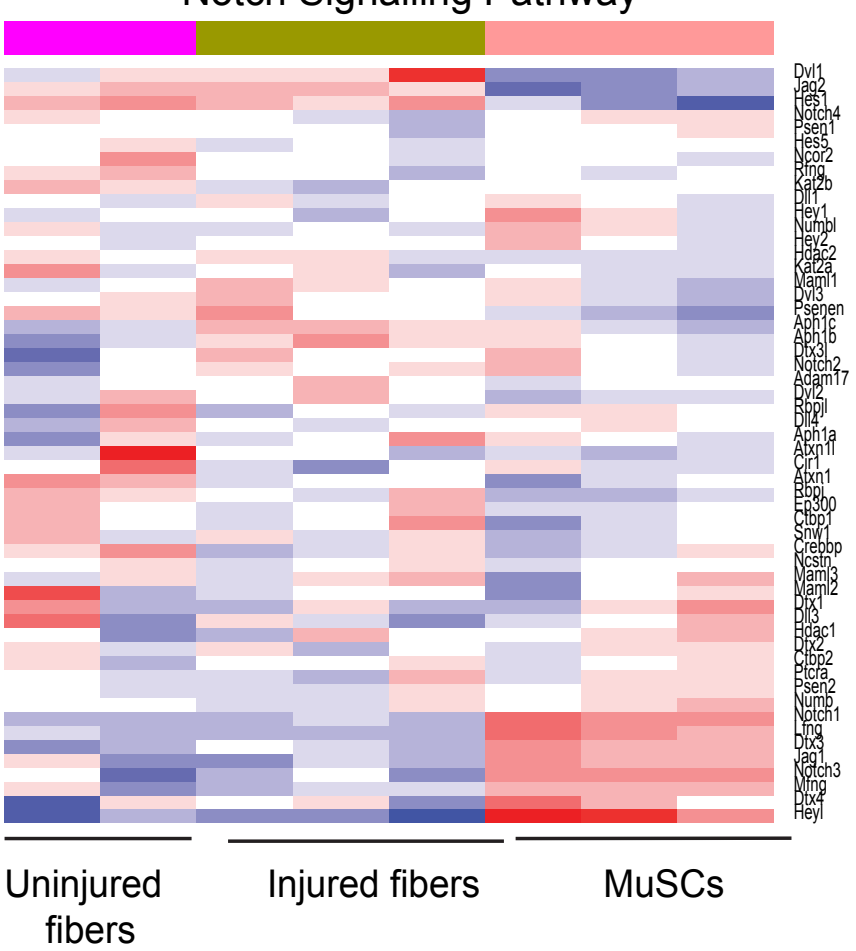

D

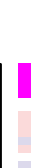

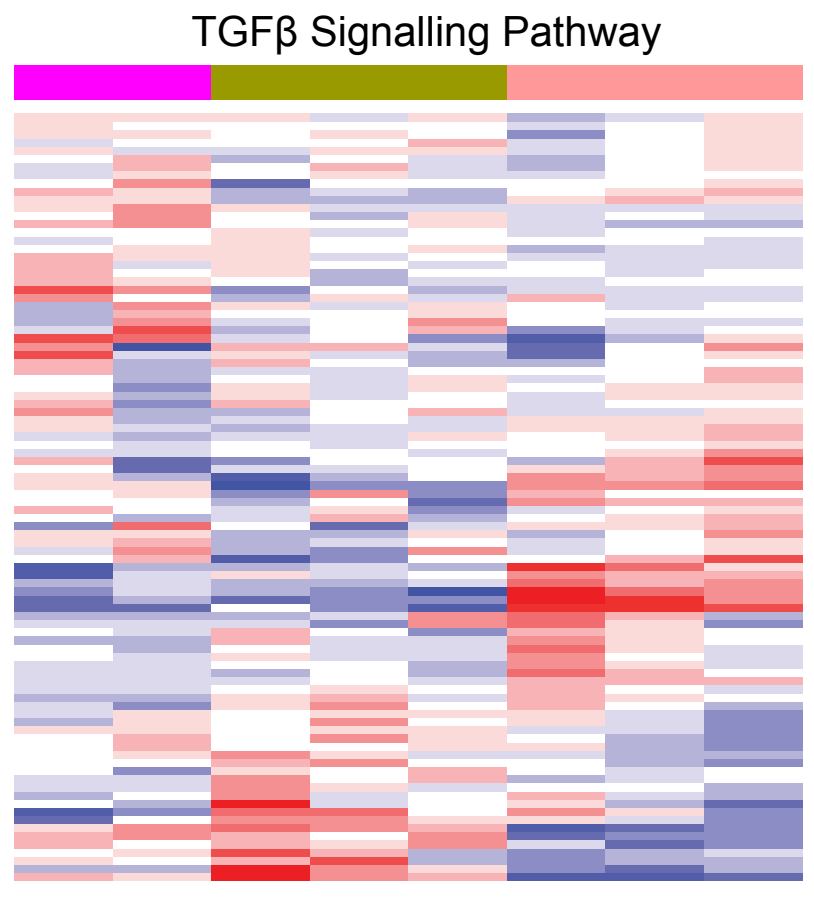

B

7 Uninjured fibers

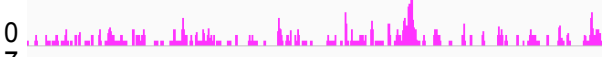
7 Injured fibers

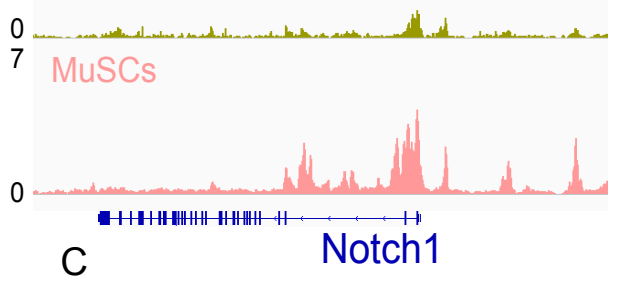

8 Uninjured fibers

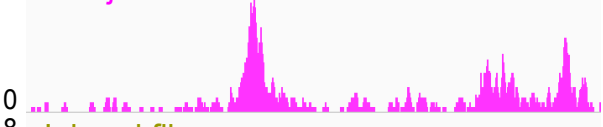

8 Injured fibers

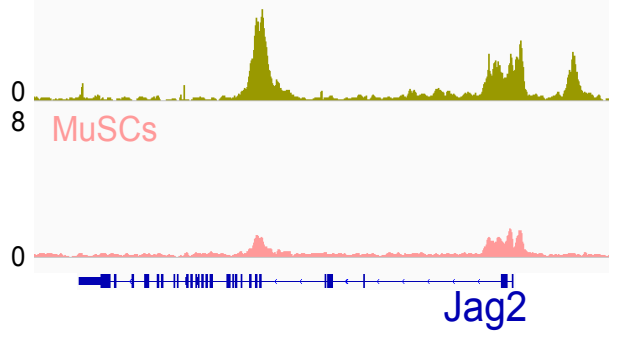

E

6 Uninjured fibers

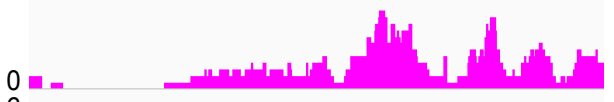

6 Injured fibers
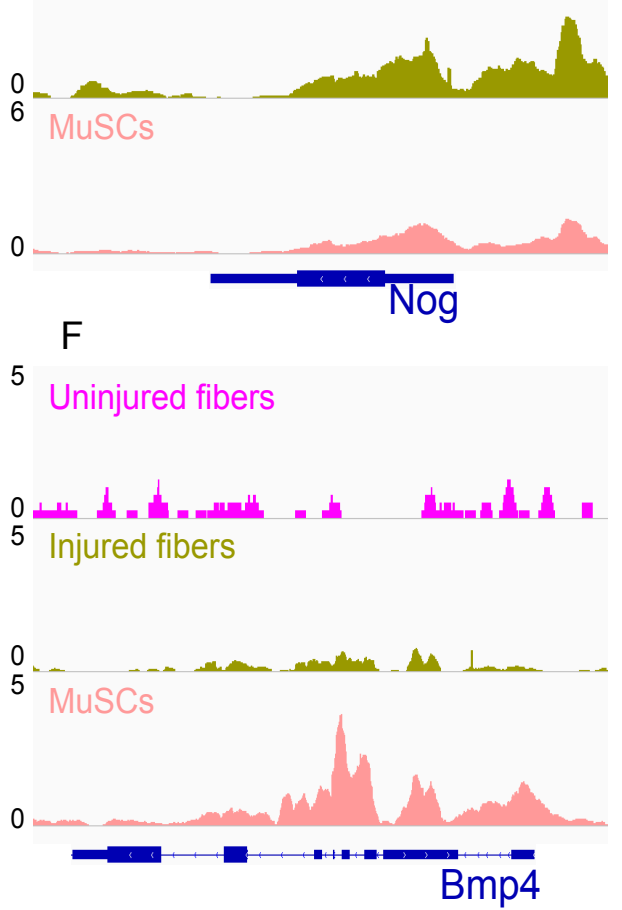
Figure 6- Figure Supplement 1

A

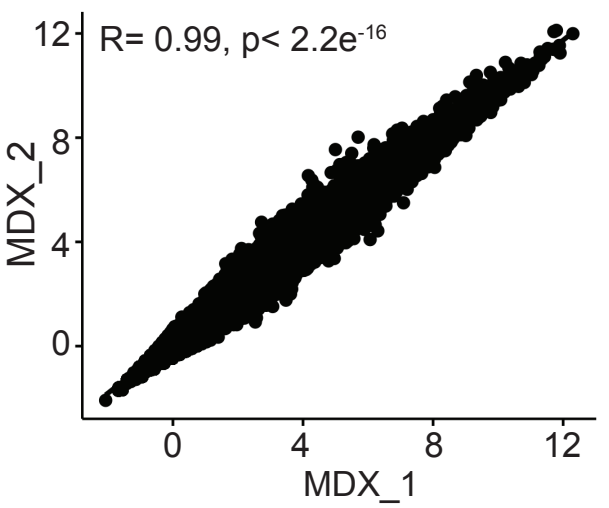

$\mathrm{D}$

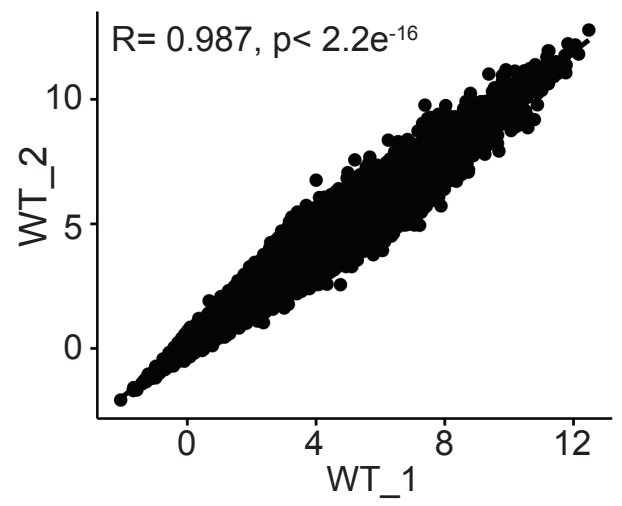

G

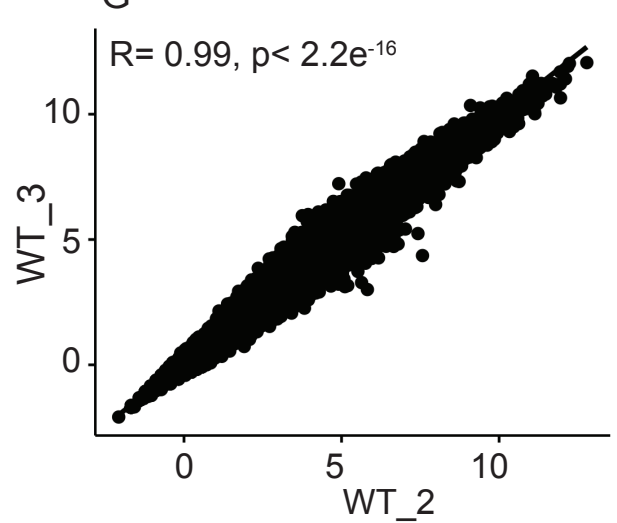

$\mathrm{B}$

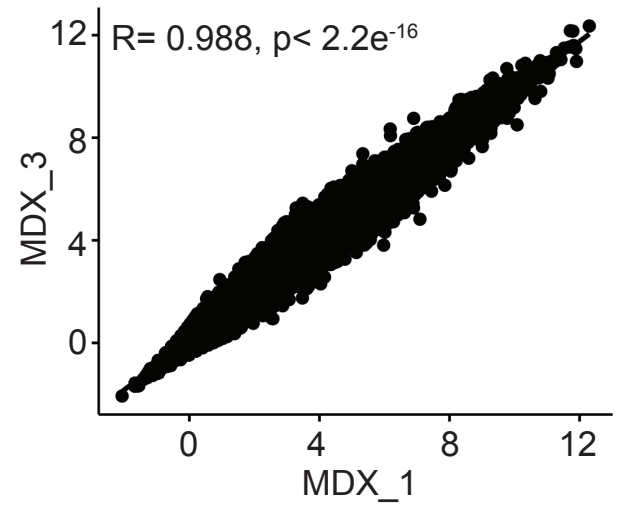

$\mathrm{E}$

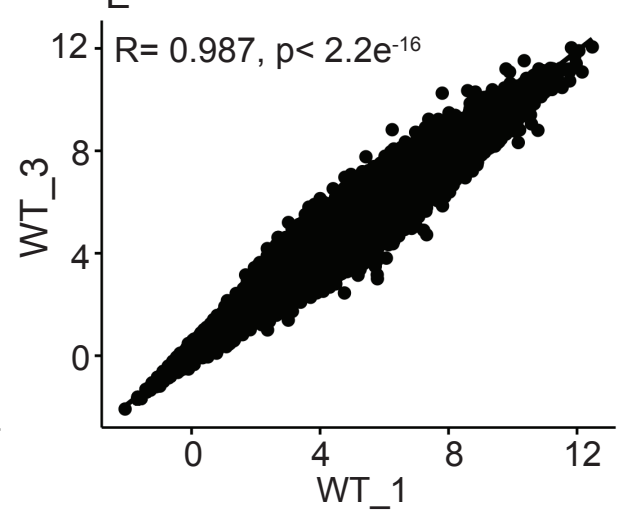

$\mathrm{H}$

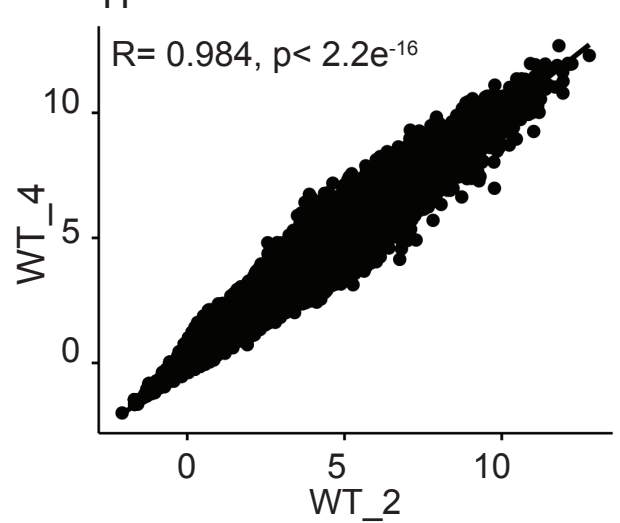

C
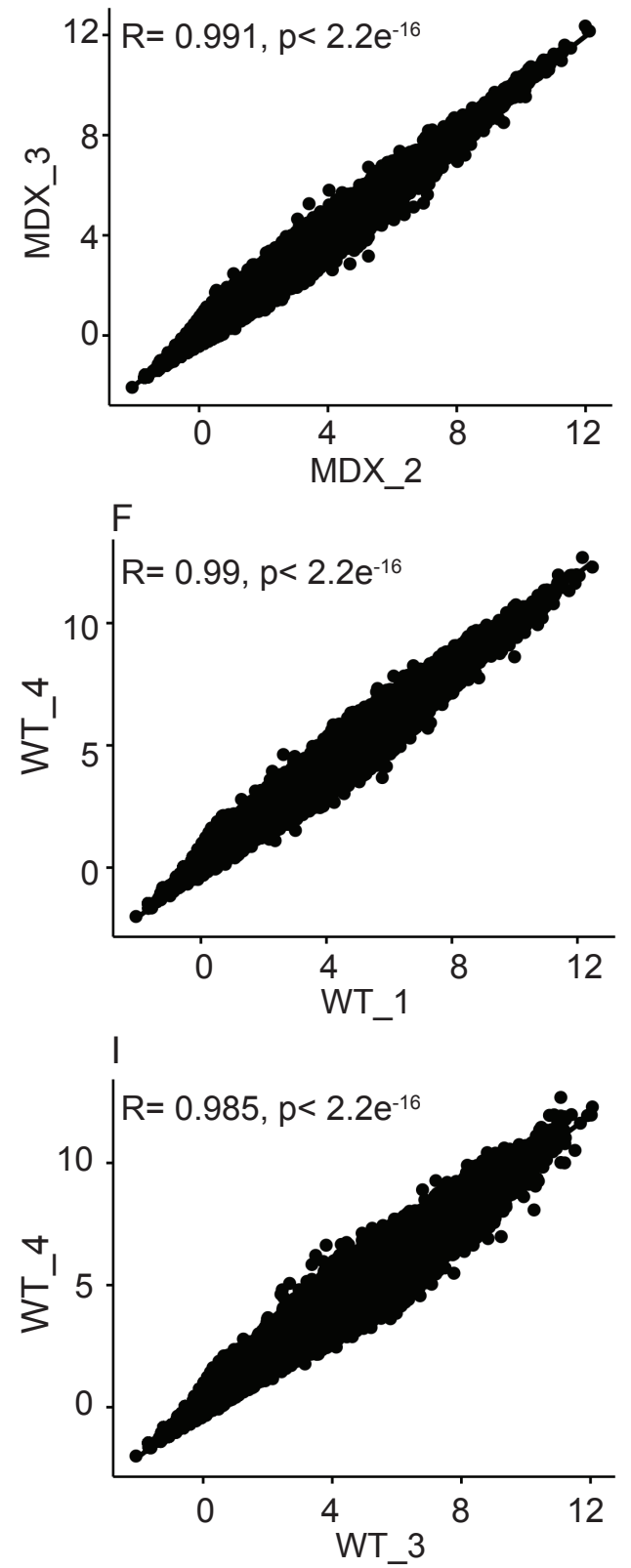
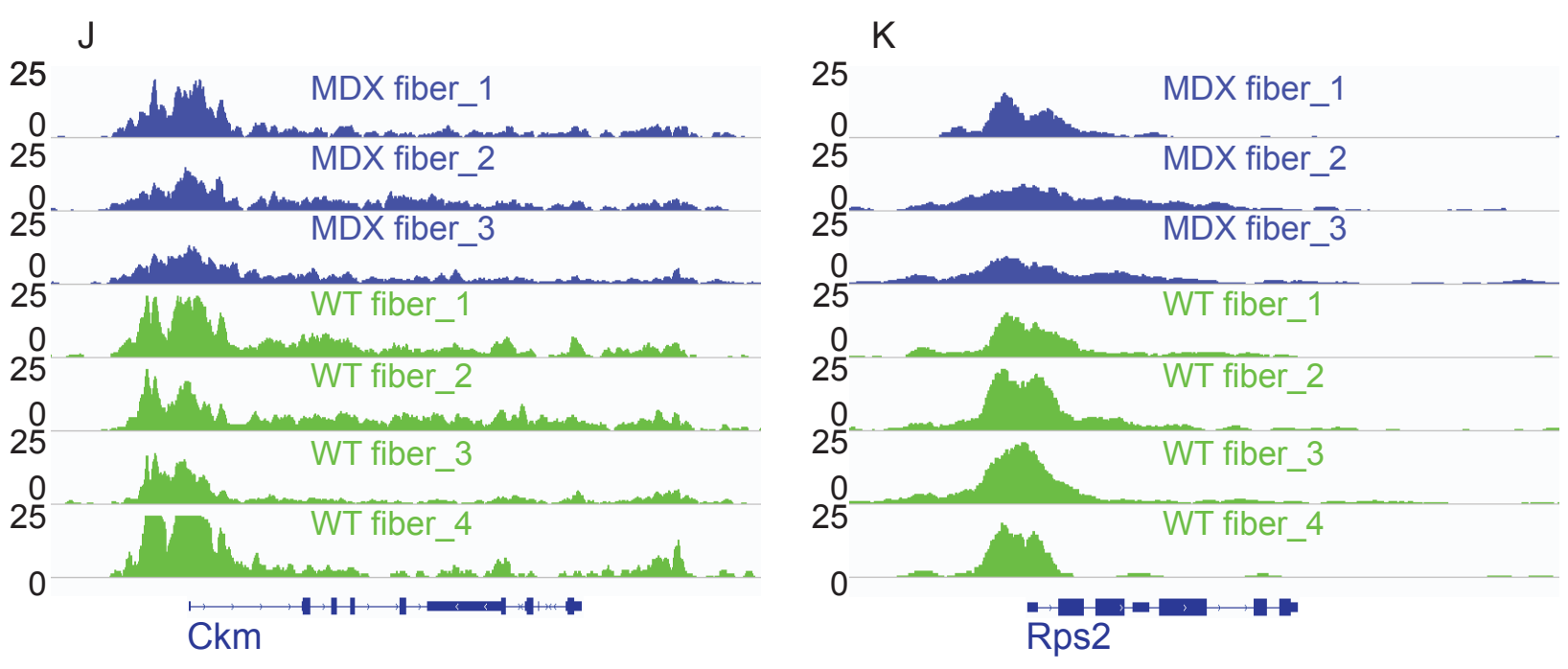
Figure 6- Figure Supplement 2

A

20 MDX fibers

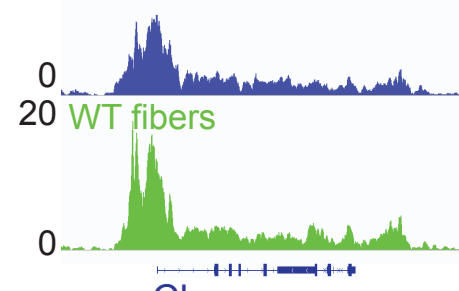

Ckm

\section{E}

25 MDX fibers
B

30 MDX fibers

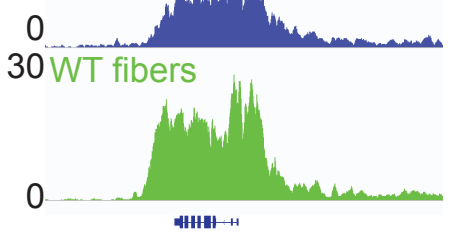

Acta1

\begin{abstract}
$\mathrm{F}$
\end{abstract}
10 MDX fibers
C

15 MDX fibers

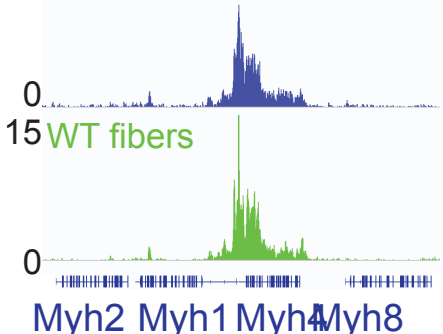

G

20 MDX fibers
D

30 MDX fibers

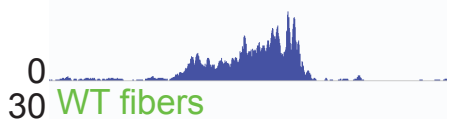

30 WT fibers

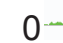

Gapdh

$\mathrm{H}$

20 MDX fibers

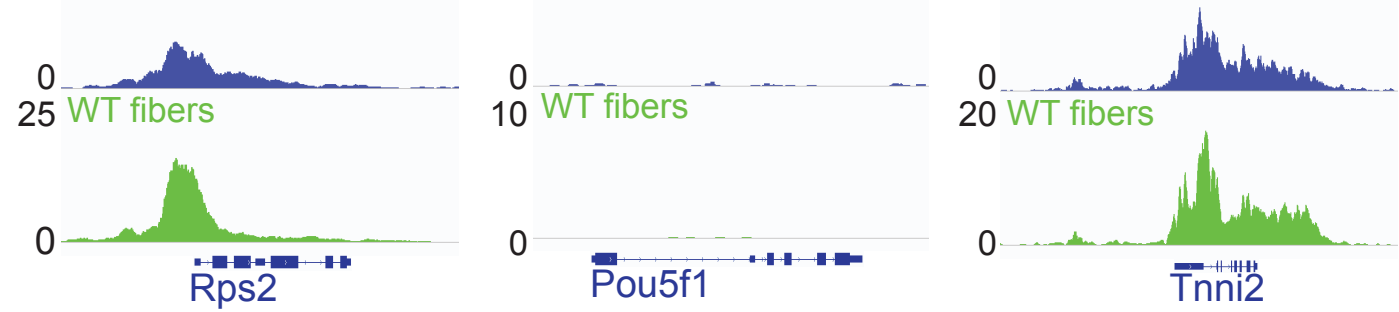

0

20 WT fibers

0

Tnnt1

Non-myogenic genes

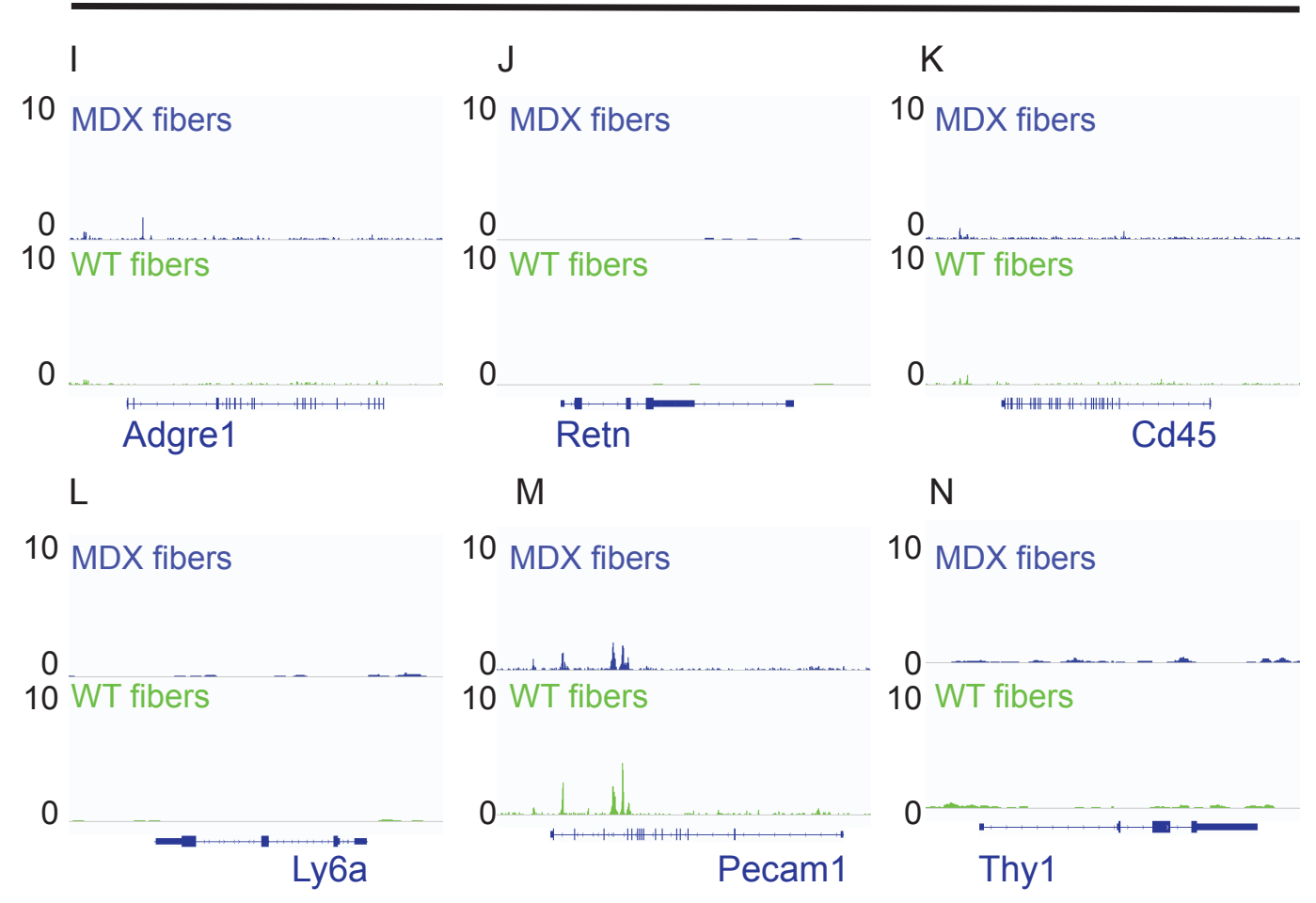


Figure 6- Figure Supplement 3

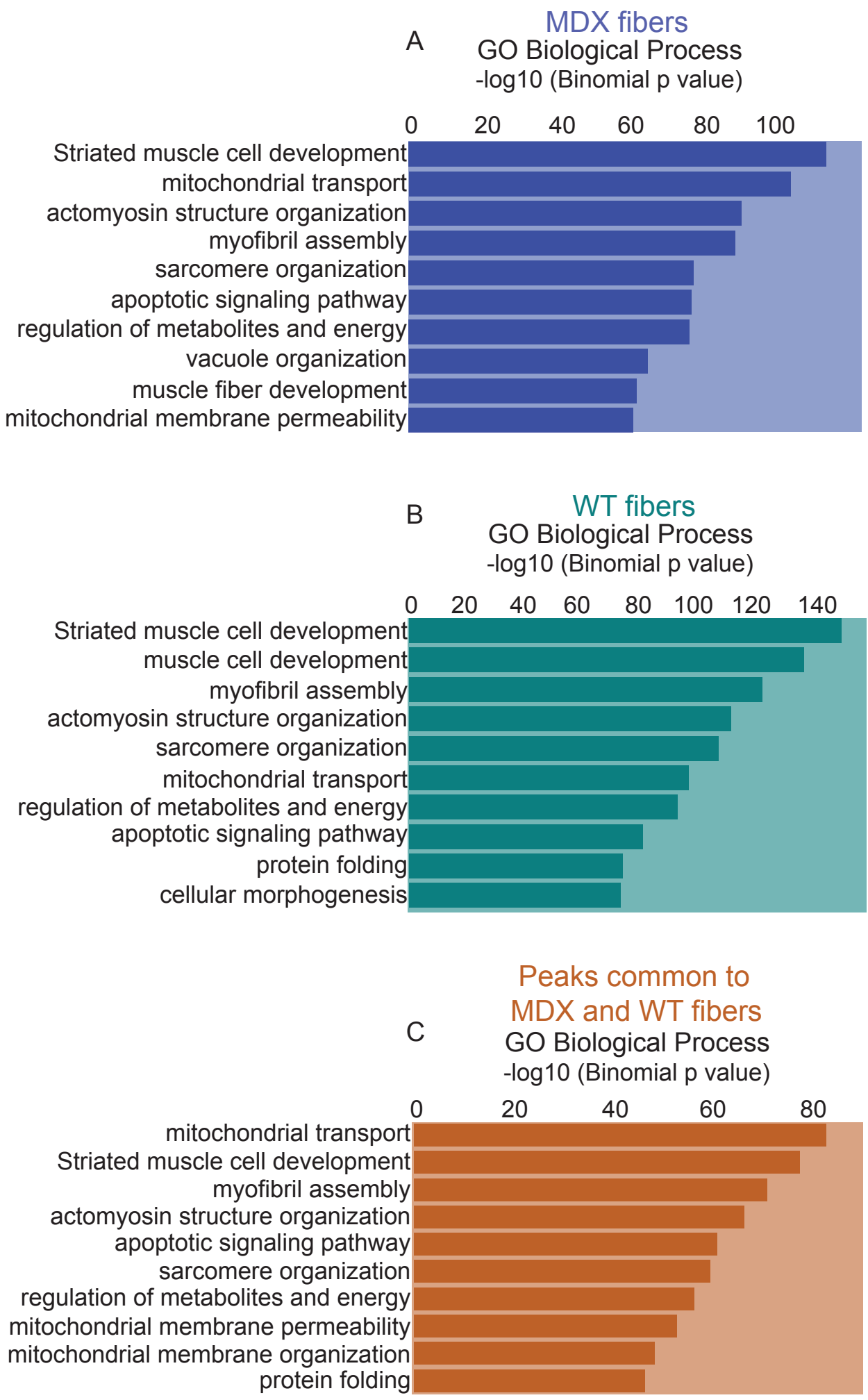


Figure 6 - Figure Supplement 4
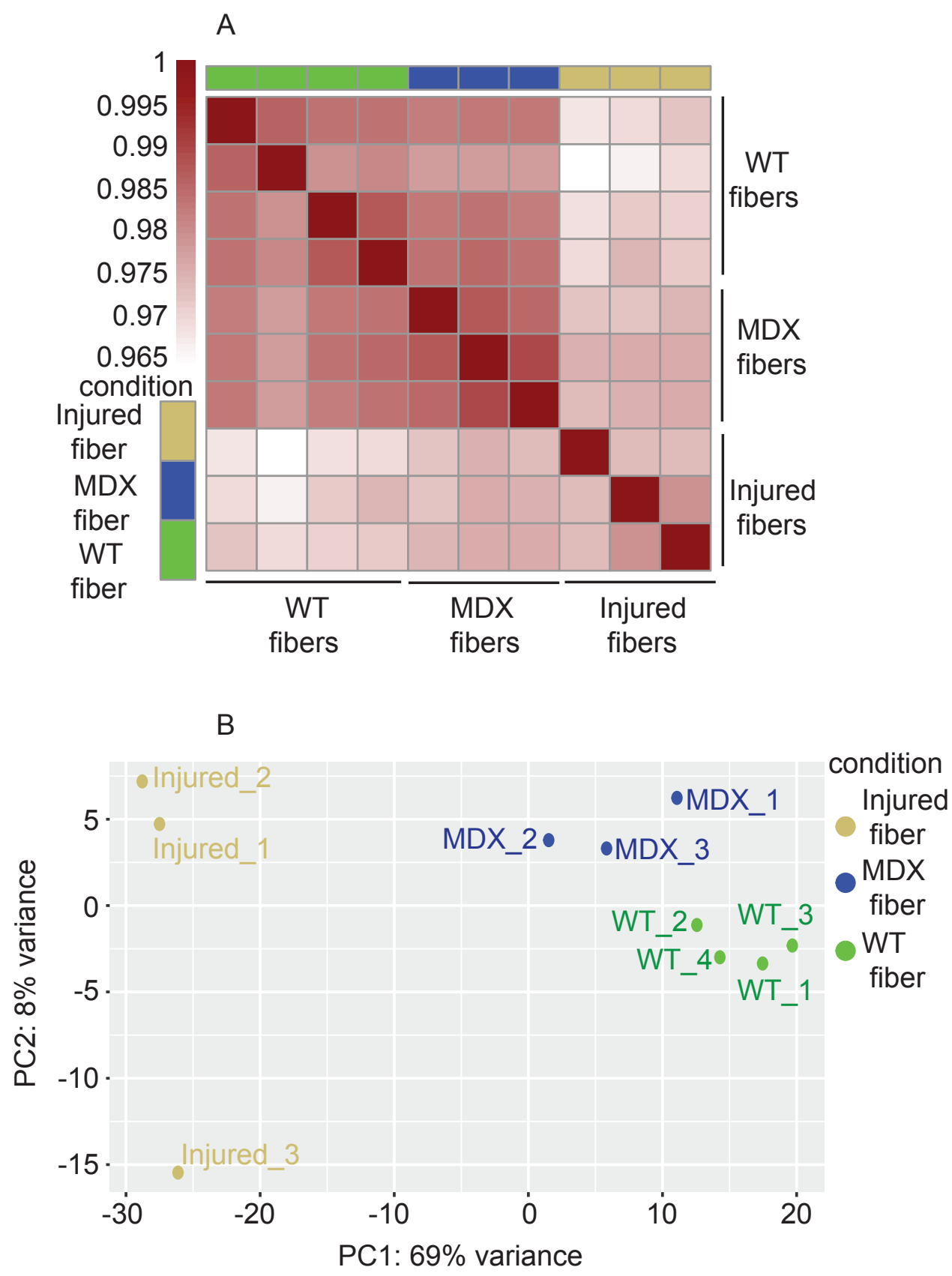
Figure 6 - Figure Supplement 5

A

Top motifs enriched in peaks common to MDX and WT Myofibers overlapping the promoters

Motif Name p-value

ATAGTCPCCCTCGTGCCA

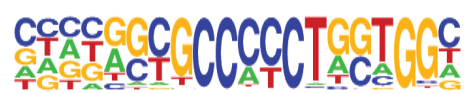

CTAAAATAG

${ }_{\mathrm{E}}^{\mathrm{G} C C C C C C C C C C C}$

ACTACAATTCCC

AACCGGAAGT

CTECCCATECGC

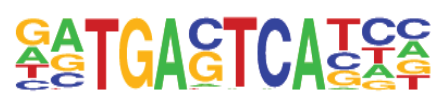

두듄TCA ATATTAC

TCCTGACTCA
CTCF $e^{-149}$

Mef2a $e^{-81}$

Sp1 $e^{-59}$

ETS $e^{-39}$

NRF1 $e^{-39}$

JUN $\quad e^{-37}$

Six1 $\quad e^{-34}$

Bach2 $e^{-27}$
BORIS $e^{-82}$

GFY $\quad \mathrm{e}^{-43}$
B

Top motifs enriched in peaks unique to MDX myofibers overlapping the promoters

Motif

C्TCATTGT 둘둘울

AAGCACTTA

Sox 10

$e^{-303}$

A.

$e^{-227}$

CAAGACATCTGTTC

PR

$e^{-202}$

CTGTTTAC

Foxo 1

$e^{-19}$

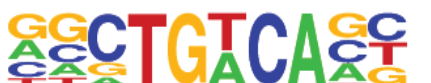

Meis 1

$e^{-189}$

프륻C.CACG

Rbpj1

$\mathrm{e}^{-174}$ 국AㅅACCCT

Eomes

$\mathrm{e}^{-169}$

CAGTCCTCCC

Znf263

$\mathrm{e}^{-168}$ CTAATTGGE

Is|1

$e^{-165}$

C

Top motifs enriched in peaks unique to WT myofibers overlapping the promoters

Motif

Name p-value

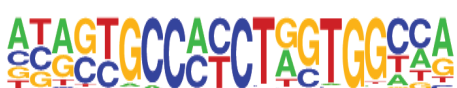

CTCF $e^{-7}$ 